\title{
Multimoment Hydrodynamics in Problem on Flow around a Sphere: Entropy Interpretation of the Appearance and Development of Instability
}

\author{
Igor V. Lebed \\ Zhukovsky Central Institute of Aerohydrodynamics, Moscow, Russia \\ Email: lebed-i@newmail.ru \\ Received 31 March 2014; revised 30 April 2014; accepted 8 May 2014 \\ Copyright (C) 2014 by author and Scientific Research Publishing Inc. \\ This work is licensed under the Creative Commons Attribution International License (CC BY). \\ http://creativecommons.org/licenses/by/4.0/

(c) (i) Open Access

\section{Abstract}

Multimoment hydrodynamics equations are applied to investigate the phenomena of appearance and development of instability in problem on a flow around a solid sphere at rest. The simplest solution to the multimoment hydrodynamics equations coincides with the Stokes solution to the classic hydrodynamics equations in the limit of small Reynolds number values, $\operatorname{Re} \ll 1$. Solution Sol $_{0}$ to the multimoment hydrodynamics equations reproduces recirculating zone in the wake behind the sphere having the form of an axisymmetric toroidal vortex ring. The $\mathrm{Sol}_{0}$ solution remains stable while the entropy production in the system exceeds the entropy outflow through the surface confining the system. The passage of the first critical value $\operatorname{Re}_{0}^{*}$ is accompanied by the Sol $_{0}$ solution stability loss. The Sol $_{0}$ solution, when loses its stability, reproduces periodic pulsations of the periphery of the recirculating zone in the wake behind the sphere. The Sol $_{1}$ and $\mathrm{Sol}_{2}$ solutions to the multimoment hydrodynamics equations interpret a vortex shedding. After the second critical value $\mathrm{Re}_{0}^{* *}$ is reached, the $\mathrm{Sol}_{0}$ solution at the periphery of the recirculating zone and in the far wake is replaced by the $\mathrm{Sol}_{2}$ solution. In accordance with the $\mathrm{Sol}_{2}$ solution, the periphery of the recirculating zone periodically detached from the core and moves downstream in the form of a vortex ring. After the attainment of the third critical value $\mathrm{Re}_{0}^{* * *}$, the $\mathrm{Sol}_{2}$ solution at the periphery of the recirculating zone and in the far wake is replaced by the Sol $_{1}$ solution. In accordance with the Sol $_{1}$ solution, vortex rings penetrate into each other and form the continuous vortex sheet in the wake behind the sphere. The replacement of one unstable flow regime by another unstable regime is governed the tendency of the system to discover the fastest 
path to depart from the state of statistical equilibrium. Having lost the stability, the system does not reach a new stable position. Such a scenario differs from the ideas of classic hydrodynamics, which interprets the development of instability in terms of bifurcations from one stable state to another stable state. Solutions to the multimoment hydrodynamics equations indicate the direction of instability development, which qualitatively reproduces the experimental data in a wide range of Re values. The problems encountered by classic hydrodynamics when interpreting the observed instability development process are solved on the way toward an increase in the number of principle hydrodynamic values.

\section{Keywords}

\section{Multimoment Hydrodynamics, Instability, Entropy, Vortex Shedding}

\section{Introduction}

Detailed evaluating the results of the direct numerical integration of the Navier-Stokes equations against experiment in problem on flow past a hard sphere at rest is carried out in [1]. Experiment records three stable medium states. Each of these three stable flows begins to develop in its own direction, qualitatively different from other flows when it loses stability. Calculation satisfactory reproduces all three stable medium states observed experimentally. However, the calculation is incapable of producing anything that corresponds to seven unstable regimes observed along the three directions of instability development. The analysis of numerous divergences between the results of numerical integration of the Navier-Stokes equations and the experiment [1]-[3] led to the following conclusion. Solutions to the classic hydrodynamics equations successfully reach the border of the instability field represented by the dashed slanting line in Figure 1 from [3]. As Reynolds number grows, these solutions move along the border of the field. However, the solutions to the classic hydrodynamics equations are unable to cross this border and to pass into the instability field.

The Navier-Stokes equations themselves are called as the most probable reason for discrepancies between calculation and experiment [1]-[4]. It may be likely that the Navier-Stokes equations become inapplicable to unstable phenomena. The responsibility for the failure of the classic hydrodynamics was laid on the approximation made in deriving the Boltzmann equation, namely, the hypothesis of molecular chaos "Stosszahlansatz". The Boltzmann hypothesis closes the kinetic equation, allowing classic hydrodynamics to be constructed for only three lower principle hydrodynamic values. It turns out that the neglect of higher principle hydrodynamic values does not introduce visually observable changes into the picture of stable flows. This error, however, grows very rapidly after the loss of stability.

In present paper the multimoment hydrodynamics equations [5] are applied to solve a problem on flow past a hard sphere at rest at a wide range of Re values. The direction of evolution of the ground axisymmetric flow $\mathbf{U}_{0}^{e x p}(\mathbf{x})$ [1] after losing its stability is studied. In Section 2, the problem is formulated. In Section 3, the problem is solved for the Stokes flow around a sphere, i.e., at $\operatorname{Re} \ll 1$. The Section 4 is devoted to construction of the $\mathrm{Sol}_{0}$ solution to the multimoment hydrodynamics equations suitable of interpreting the stationary axisymmetric flow at a moderately high Re values. The Section 5 examines the behavior of the $\mathrm{Sol}_{0}$ solution that loses its stability after the passage of the first critical Reynolds number value $\operatorname{Re}_{0}^{*}$. In Section 6 , the characteristic features of the appearance of instability are interpreted in terms of entropy. The principle of retention and loss of the open system stability is formulated. In Section 7, the characteristic features of the development of instability are interpreted in terms of entropy. The evolution criterion is formulated. The Section 8 is devoted to finding the solutions to the multimoment hydrodynamics equations capable of reproducing a vortex shedding. The Section 9 provides an algorithm to select one unstable solution of many found unstable solutions. The selected solution indicates the direction of system evolution. In Section 10, the results are compared with the experimental data.

\section{Problem Statement}

Consider a space filled with thermodynamically equilibrium gas. Suppose that a solid sphere of radius $a$ 
moves in gas at constant velocity $\mathbf{U}_{0}$ along the $Z_{0}$ axis of the immobile Cartesian frame of reference $X_{0} Y_{0} Z_{0}$. Let us now pass from $X_{0} Y_{0} Z_{0}$ to the Cartesian frame of reference $X Y Z$ with the axes parallel to those of $X_{0} Y_{0} Z_{0}$ and the origin made coincident with the center of the moving sphere. In the $X Y Z$ frame of reference, the sphere is at rest, the inflowing-gas velocity at an infinite distance from the sphere, $\mathbf{U}_{0}$, is aligned with the positive direction of the $Z$ axis, and the flow problem is stationary.

The pair distribution function $f_{p}^{(0)}\left(\mathbf{G}_{0}, \mathbf{v}_{0}\right)$ corresponding to the equilibrium state is

$$
f_{p}^{(0)}\left(\mathbf{G}_{0}, \mathbf{v}_{0}\right)=\frac{n_{0}}{2}\left(\frac{M}{2 \pi k T_{0}}\right)^{3 / 2}\left(\frac{\mu}{2 \pi k T_{0}}\right)^{3 / 2} \exp \left[-\frac{M \mathbf{G}_{0}^{2}}{2 k T_{0}}-\frac{\mu \mathbf{v}_{0}^{2}}{2 k T_{0}}\right]
$$

where $n_{0}$ and $T_{0}$ are the local density of the number of particles and the temperature of unperturbed gas, $M=2 m, \mu=m / 2, m$ is the mass of a gas particle, and $\mathbf{G}_{0}$ and $\mathbf{v}_{0}$ are the velocity of the center of mass and relative velocity of the pair of particles. In $X Y Z$, the pair distribution function $f_{p}(\mathbf{x}, \mathbf{G}, v)$ can be written as

$$
\begin{gathered}
f_{p}(\mathbf{x}, \mathbf{G}, v)=f_{p}^{(0)}(\mathbf{G}, v)+\Delta f_{p}(\mathbf{x}, \mathbf{G}, v) \\
f_{p}^{(0)}(\mathbf{G}, v)=2 \pi n_{0}\left(\frac{M}{2 \pi k T_{0}}\right)^{3 / 2}\left(\frac{\mu}{2 \pi k T_{0}}\right)^{3 / 2} \exp \left[-\frac{M\left(\mathbf{G}-\mathbf{U}_{0}\right)^{2}}{2 k T_{0}}-\frac{\mu v^{2}}{2 k T_{0}}\right] \\
\mathbf{G}=\mathbf{G}_{0}+\mathbf{U}_{0} \quad \mathbf{v}=\mathbf{v}_{0} \quad v=|\mathbf{v}|
\end{gathered}
$$

Let $x, y$, and $z$ be the Cartesian coordinates of point $\mathbf{x}$ in the space; $r, \theta$, and $\varphi$ are its spherical coordinates, $x=r \sin \theta \cos \varphi, y=r \sin \theta \sin \varphi$, and $z=r \cos \theta$, Figure 1. Bring the origin of the Cartesian frame of

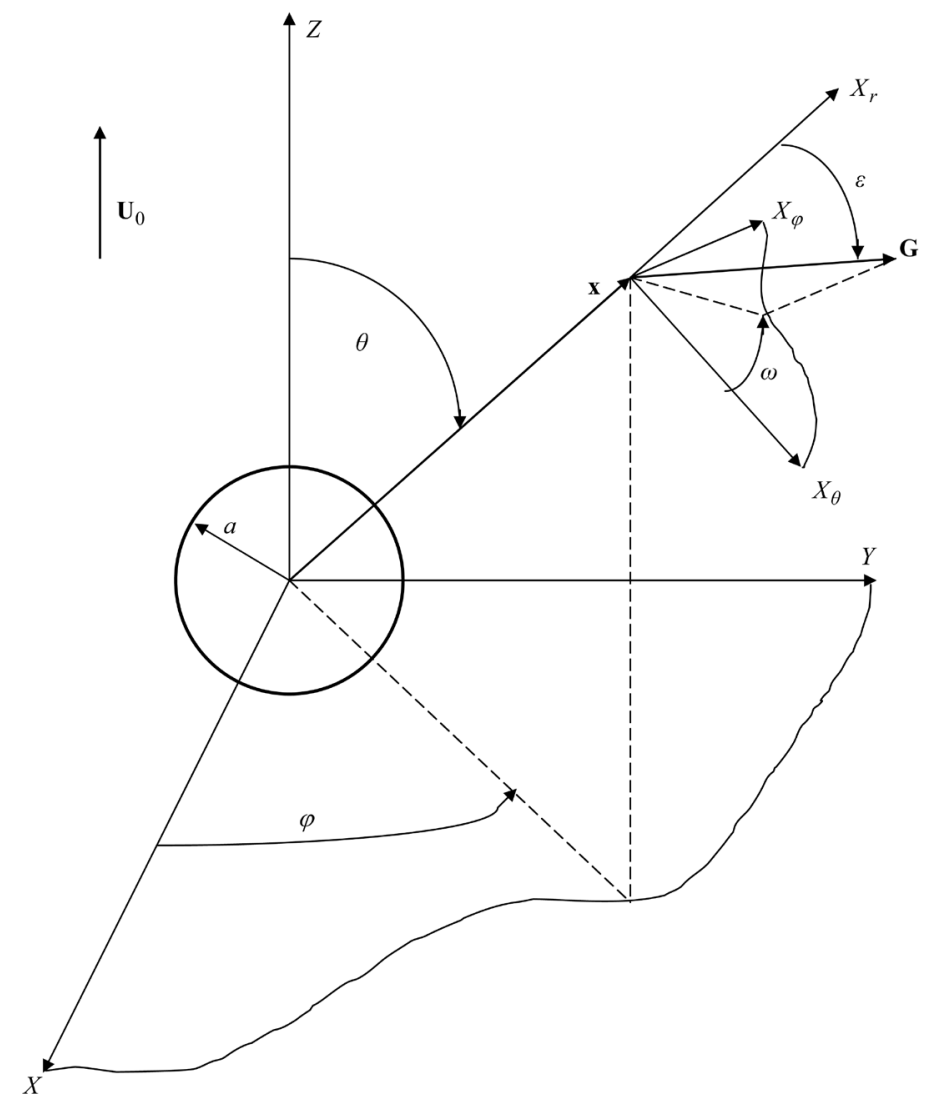

Figure 1. The $X Y Z$ frame with its origin at the center of the sphere. The $X_{r} X_{\theta} X_{\varphi}$ frame with its origin at some point $\mathbf{x}$ in the gas. 
reference $X_{r} Y_{\theta} Z_{\varphi}$ into coincidence with point $\mathbf{x}$ so that the $X_{r}$ axis is directed along $\mathbf{x}$ vector. Mark the projections of vector $\mathbf{G}$ onto the $X Y Z$ axes by $G_{x}, G_{y}$, and $G_{z}$, its projections onto the axes of $X_{r} Y_{\theta} Z_{\varphi}$, by $G_{r}, G_{\theta}$, and $G_{\varphi}$, and its spherical coordinates, by $G, \varepsilon$, and $\omega$, Figure 1 .

The basic property of pair distribution functions (17) from [3] is that $f_{p}(t, \mathbf{x}, \mathbf{G}, v)$ is conserved with time along the trajectory of the center of mass of a pair of particles. In the stationary case, the $f_{p}(\mathbf{x}, \mathbf{G}, v)$ function remains unchanged along a straight line parallel to vector $\mathbf{G}$

$$
\mathbf{G} \frac{\partial f_{p}(\mathbf{x}, \mathbf{G}, v)}{\partial \mathbf{x}}=0
$$

The $\mathrm{F}_{x y}=G_{x} y-G_{y} x, \mathrm{~F}_{z x}=G_{z} x-G_{x} z$, and $\mathrm{F}_{z y}=G_{z} y-G_{y} z$ functions are the first integrals of Equation (2.3). These functions were termed in [6] trajectory invariants. In this study, consideration is restricted to gas flows around a sphere, which are invariant under rotation through an arbitrary angle $\varphi$ about the $Z$ axis. Let us compose combinations of trajectory invariants $\mathrm{F}_{x y}, \mathrm{~F}_{z x}$, and $\mathrm{F}_{z y}$, invariant with respect to this rotation

$$
\begin{gathered}
\mathrm{F}_{x y}=-G_{r} \sin \theta \sin \varepsilon \sin \omega \\
\Phi_{z}=-\left(G_{x} \mathrm{~F}_{z x}+G_{y} \mathrm{~F}_{z y}\right)=G^{2} r\left(\cos \theta \sin ^{2} \varepsilon+\sin \theta \cos \varepsilon \sin \varepsilon \cos \omega\right) \\
\Phi_{r}=\left(\mathrm{F}_{x y}^{2}+\mathrm{F}_{z x}^{2}+\mathrm{F}_{z y}^{2}\right)^{1 / 2}=G r \sin \varepsilon
\end{gathered}
$$

By virtue of the flow geometry, there are two independent regions of integration with respect to $\mathbf{G}$ at each point $\mathbf{x}$ in the gas. The first region, denote it by $W_{1}$, is a spherical cone, Figure 2. This region incorporates the trajectories of centers of mass of pairs of particles that originate and terminate at the surface of the sphere. The integration limits for this group of trajectories are $0 \leq G \leq \infty, 0 \leq \omega \leq 2 \pi, 0 \leq \varepsilon \leq \arcsin (a / r)$. The second region, $W_{2}$, embraces all possible trajectories of centers of mass of particle pairs converging to point $\mathbf{x}$. The integration limits for the second group of trajectories are unbounded.

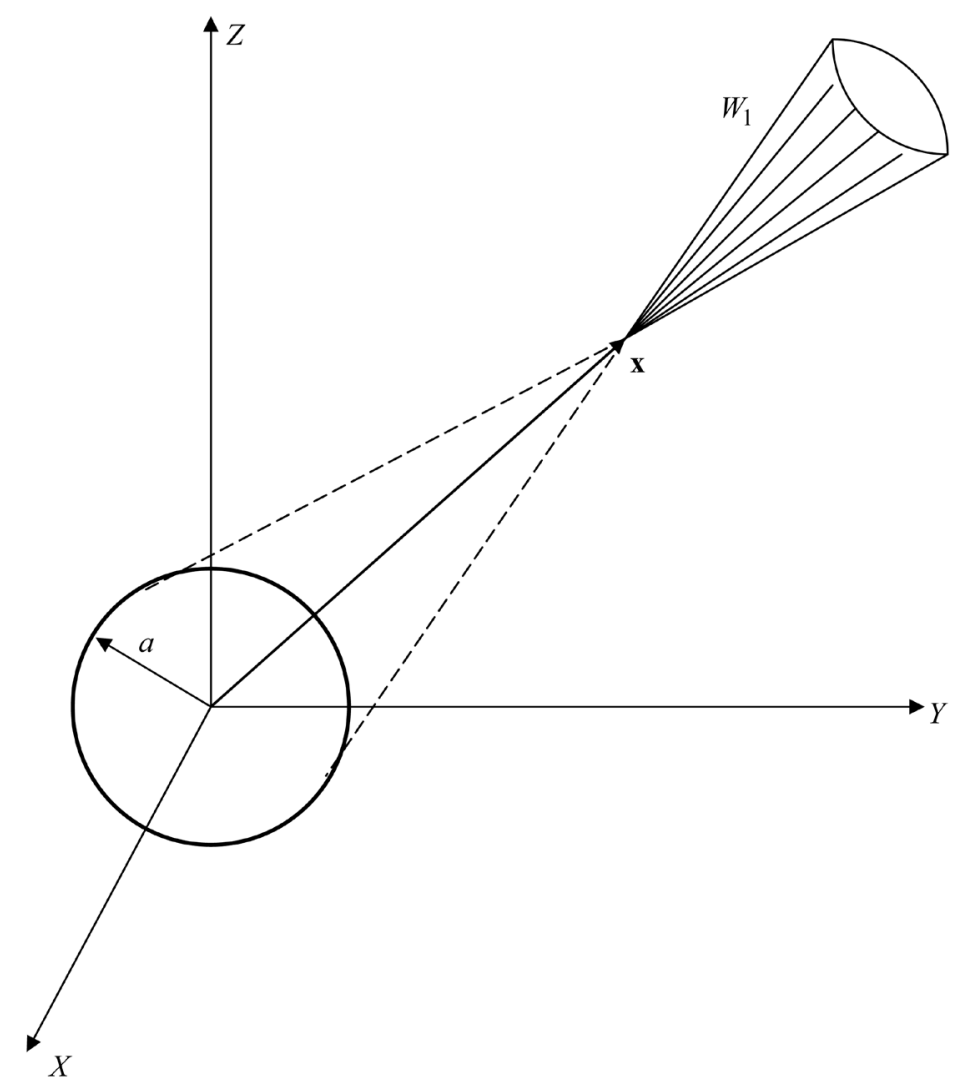

Figure 2. Regions of integration with respect to $\mathbf{G}$. 
According to the general approach to solving the multimoment hydrodynamic equations in terms of pair functions, outlined in [6], solution $\Delta f_{p}(\mathbf{x}, \mathbf{G}, v)$ is sought for in the form of an infinite series of the products of trajectory invariants (2.4)

$$
\Delta f_{p}(\mathbf{x}, \mathbf{G}, v)=4 \pi\left(\frac{M}{2 \pi k T_{0}}\right)^{3 / 2}\left(\frac{\mu}{2 \pi k T_{0}}\right)^{3 / 2} \exp \left[-\frac{M \mathbf{G}^{2}}{2 k T_{0}}-\frac{\mu v^{2}}{2 k T_{0}}\right] \sum_{k=0}^{+\infty} \sum_{l=-\infty}^{+\infty} \sum_{m=0}^{+\infty} \sum_{n=0}^{+\infty} c_{k l m n}(\mathbf{G}, v) G_{z}^{k} \Phi_{r}^{l} \Phi_{z}^{m} F_{x y}^{n}
$$

The spatial dependence of $\Delta f_{p}(\mathbf{x}, \mathbf{G}, v)$ (2.5) is controlled by trajectory invariants (2.4), and coefficients $c_{k l m n}$ are independent of $\mathbf{x}$. In keeping with definitions given in [5], the expressions for the principal hydrodynamic values - the moments of $f_{p}(\mathbf{x}, \mathbf{G}, v)(2.2)$ — can be written as

$$
\begin{gathered}
\frac{1}{2} n=\frac{1}{2} n_{0}+\int \Delta f_{p} \mathrm{~d} \mathbf{g}, \\
\frac{1}{2} n U_{i}=\frac{1}{2} n_{0} U_{0 i}+\int G_{i} \Delta f_{p} \mathrm{~d} \mathbf{g}, \\
\frac{3}{4} p^{v}=\frac{3}{4} n_{0} k T_{0}+\int \frac{\mu v^{2}}{2} \Delta f_{p} \mathrm{~d} \mathbf{g} \\
\frac{3}{4} p^{G}=\frac{3}{4} s^{G}+\frac{1}{4} M n_{0} U_{0}^{2}-\frac{1}{4} M n U^{2}, \frac{3}{4} s^{G}=\frac{3}{4} n_{0} k T_{0}+\int \frac{M G^{2}}{2} \Delta f_{p} \mathrm{~d} \mathbf{g}, \\
p^{v}=n k T^{v}, p^{G}=n k T^{G}, \\
\frac{1}{2} P_{i j}^{G}=\frac{1}{2} S_{i j}^{G}+\frac{1}{2} M n_{0} U_{0 i} U_{0 j}-\frac{1}{2} M n U_{i} U_{j}, \quad \frac{1}{2} S_{i j}^{G}=\frac{1}{2} n_{0} k T_{0} \delta_{i j}+\int M G_{i} G_{j} \Delta f_{p} \mathrm{~d} \mathbf{g}, \\
p_{i j}^{G}=P_{i j}^{G}-p^{G} \delta_{i j}, \quad s_{i j}^{G}=S_{i j}^{G}-s^{G} \delta_{i j}, \\
\frac{1}{2} q_{i}^{v}=\frac{3}{4} n_{0} k T_{0} U_{0 i}+\frac{1}{2} Q_{i}^{v}-\frac{3}{4} p^{v} U_{i}, \quad \frac{1}{2} Q_{i}^{v}=\int \frac{\mu G_{i} v^{2}}{2} \Delta f_{p} \mathrm{~d} \mathbf{g}, \\
\frac{1}{2} q_{i}^{G}=\frac{5}{4} n_{0} k T_{0} U_{0 i}+\frac{1}{4} M n_{0} U_{0 i} U_{0}^{2}+\frac{1}{2} Q_{i}^{G}-\frac{5}{4} p^{G} U_{i}-\frac{1}{2} p_{k i}^{G} U_{k}-\frac{1}{4} M n U_{i} U^{2}, \frac{1}{2} Q_{i}^{G}=\int \frac{M G_{i} G^{2}}{2} \Delta f_{p} \mathrm{~d} \mathbf{g}
\end{gathered}
$$

Here, $n$ is the local density of the number of particles; $\mathbf{U}$ is the hydrodynamic velocity; $p^{G}, T^{G}, P_{i j}^{G}$, and $\mathbf{q}^{G}$ are the pressure, temperature, stress tensor, and heat flux corresponding to motion of the centers of mass of pairs of particles; $p^{v}, T^{v}$, and $\mathbf{q}^{v}$ correspond to relative motion of particles within pairs; $\mathrm{d} \mathbf{g}=v^{2} \mathrm{~d} v \mathrm{~d} \mathbf{G}$.

Nonprincipal hydrodynamic values $p_{i j}^{v}$ and $\mathbf{q}^{G v}$ are given by Equation (21) from [3] with the NavierStokes accuracy. In terms of spherical coordinates, these values for cylindrically symmetric flows considered in this study (i.e., for flows invariant with respect to rotation through an arbitrary angle $\varphi$ about the $Z$ axis) assume the form

$$
\begin{gathered}
p_{r r}^{v}=-2 \tilde{\eta} \frac{\partial U_{r}}{\partial r}-\frac{4 \tilde{\eta}}{3 p^{v}} \frac{\partial q_{r}^{v}}{\partial r}+\pi^{v}=-\frac{4 \tilde{\eta}}{3 p^{v}} \frac{\partial Q_{r}^{v}}{\partial r} \\
p_{\theta \theta}^{v}=-2 \tilde{\eta}\left(\frac{U_{r}}{r}+\frac{\partial U_{\theta}}{r \partial \theta}\right)-\frac{4 \tilde{\eta}}{3 p^{v}}\left(\frac{q_{r}^{v}}{r}+\frac{\partial q_{\theta}^{v}}{r \partial \theta}\right)+\pi^{v}=-\frac{4 \tilde{\eta}}{3 p^{v}}\left(\frac{Q_{r}^{v}}{r}+\frac{\partial Q_{\theta}^{v}}{r \partial \theta}\right) \\
p_{\varphi \varphi}^{v}=-2 \tilde{\eta}\left(\frac{U_{r}}{r}+\frac{U_{\theta} \operatorname{ctg} \theta}{r}\right)-\frac{4 \tilde{\eta}}{3 p^{v}}\left(\frac{q_{r}^{v}}{r}+\frac{q_{\theta}^{v} \operatorname{ctg} \theta}{r}\right)+\pi^{v}=-\frac{4 \tilde{\eta}}{3 p^{v}}\left(\frac{Q_{r}^{v}}{r}+\frac{Q_{\theta}^{v} \operatorname{ctg} \theta}{r}\right) \\
p_{r \varphi}^{v}=-\tilde{\eta}\left[r \frac{\partial}{\partial r}\left(\frac{U_{\theta}}{r}\right)+\frac{\partial U_{r}}{r \partial \theta}\right]-\frac{2 \tilde{\eta}}{3 p^{v}}\left[r \frac{\partial}{\partial r}\left(\frac{q_{\theta}^{v}}{r}\right)+\frac{\partial q_{r}^{v}}{r \partial \theta}\right]=-\frac{2 \tilde{\eta}}{3 p^{v}}\left[r \frac{\partial}{\partial r}\left(\frac{Q_{\theta}^{v}}{r}\right)+\frac{\partial Q_{r}^{v}}{r \partial \theta}\right] \\
\pi^{v}=\frac{2 \tilde{\eta}}{3} \operatorname{div} \mathbf{U}+\frac{4 \tilde{\eta}}{9 p^{v}} \operatorname{divq}^{v}
\end{gathered}
$$




$$
\begin{aligned}
& q_{r}^{G v}=-\frac{5 \tilde{\eta}}{4} \frac{\partial}{\partial r}\left(\frac{k T}{m}\right)-\frac{\tilde{\eta}}{4 p^{v} r^{2} \sin \theta}\left[\frac{\partial}{\partial r}\left(r^{2} \sin \theta \frac{k T^{v} p_{r r}^{G}}{m}\right)+\frac{\partial}{\partial \theta}\left(r \sin \theta \frac{k T^{v} p_{r \theta}^{G}}{m}\right)\right] \\
& +\frac{\tilde{\eta}}{4 p^{v} r} \frac{k T^{v}}{m}\left(p_{\theta \theta}^{G}+p_{\varphi \varphi}^{G}\right)-\frac{\tilde{\eta}}{3 p^{v}}\left(3 q_{r}^{v} \frac{\partial U_{r}}{\partial r}+q_{r}^{v} \frac{\partial U_{\theta}}{r \partial \theta}+q_{\theta}^{v} \frac{\partial U_{r}}{r \partial \theta}+q_{\theta}^{v} \frac{\partial U_{\theta}}{\partial r}+2 q_{r}^{v} \frac{U_{r}}{r}+q_{r}^{v} \frac{U_{\theta} \operatorname{ctg} \theta}{r}-q_{\theta}^{v} \frac{U_{\theta}}{r}\right) \\
& q_{\theta}^{G v}=-\frac{5 \tilde{\eta}}{4} \frac{\partial}{r \partial \theta}\left(\frac{k T}{m}\right)-\frac{\tilde{\eta}}{4 p^{v} r^{2} \sin \theta}\left[\frac{\partial}{\partial r}\left(r^{2} \sin \theta \frac{k T^{v} p_{r \theta}^{G}}{m}\right)+\frac{\partial}{\partial \theta}\left(r \sin \theta \frac{k T^{v} p_{\theta \theta}^{G}}{m}\right)\right] \\
& +\frac{\tilde{\eta}}{4 p^{v} r} \frac{k T^{v}}{m}\left(p_{\varphi \varphi}^{G} \operatorname{ctg} \theta-p_{r \theta}^{G}\right)-\frac{\tilde{\eta}}{3 p^{v}}\left(3 q_{\theta}^{v} \frac{\partial U_{\theta}}{r \partial \theta}+q_{\theta}^{v} \frac{\partial U_{r}}{\partial r}+q_{r}^{v} \frac{\partial U_{\theta}}{\partial r}+q_{r}^{v} \frac{\partial U_{r}}{r \partial \theta}+q_{\theta}^{v} \frac{U_{\theta} \operatorname{ctg} \theta}{r}+4 q_{\theta}^{v} \frac{U_{r}}{r}-q_{r}^{v} \frac{U_{\theta}}{r}\right)
\end{aligned}
$$

Here, $\tilde{\eta}$ is the coefficient of the dynamic viscosity. From the definitions of gas pressure $p$, temperature $T$, viscous stress tensor $p_{i j}$, and heat flux $\mathbf{q}$ [5] it follows that they are linear combinations of moments (2.6) and (2.7)

$$
p=\frac{p^{G}+p^{v}}{2} \quad T=\frac{T^{G}+T^{v}}{2} \quad p=n k T \quad p_{i j}=\frac{p_{i j}^{G}+p_{i j}^{v}}{2} \quad q_{i}=\frac{q_{i}^{G}}{2}+\frac{5 q_{i}^{v}}{6}+q_{i}^{G v}
$$

According to [5], the overall equations of conservation of the number of particles, momentum, and energy assume the form

$$
\begin{gathered}
\frac{\partial n}{\partial t}+\frac{\partial n U_{i}}{\partial x_{i}}=0 \\
\frac{\partial n U_{i}}{\partial t}+\frac{1}{2 m}\left(\frac{\partial S_{i j}^{G}}{\partial x_{j}}+\prod_{i}^{v}\right)=0 \\
\frac{\partial}{\partial t}\left(\frac{3 s^{G}}{4}+\frac{3 p^{v}}{4}\right)+\frac{\partial}{\partial x_{i}}\left(\frac{Q_{i}^{G}}{2}+\frac{5 Q_{i}^{v}}{6}\right)+\frac{\Lambda^{G v}}{2}=0
\end{gathered}
$$

The basic property of pair distribution functions (17) from [3] subdivides Equations (2.10) and (2.11). As recast in terms of the spherical coordinates, the $\prod_{i}^{v}$ and $\Lambda^{G v}$ components assume the following form for the cylindrically symmetric flow in question

$$
\begin{gathered}
\Pi_{r}^{v}=\frac{\partial p^{v}}{\partial r}+\frac{\partial p_{r r}^{v}}{\partial r}+\frac{\partial p_{r \theta}^{v}}{r \partial \theta}+\frac{1}{r}\left[2 p_{r r}^{v}-p_{\theta \theta}^{v}-p_{\varphi \varphi}^{v}+p_{r \theta}^{v} \operatorname{ctg} \theta\right]=0 \\
\Pi_{\theta}^{v}=\frac{\partial p^{v}}{r \partial \theta}+\frac{\partial p_{\theta \theta}^{v}}{r \partial \theta}+\frac{\partial p_{r \theta}^{v}}{\partial r}+\frac{1}{r}\left[\left(p_{\theta \theta}^{v}-p_{\varphi \varphi}^{v}\right) \operatorname{ctg} \theta+3 p_{r \theta}^{v}\right]=0 \\
\Lambda^{G v}=\frac{1}{r^{2} \sin \theta}\left\{\frac{\partial}{\partial r}\left[r^{2} \sin \theta\left(2 q_{r}^{G v}+U_{r} p_{r r}^{v}+U_{\theta} p_{r \theta}^{v}\right)\right]+\frac{\partial}{\partial \theta}\left[r \sin \theta\left(2 q_{\theta}^{G v}+U_{r} p_{r \theta}^{v}+U_{\theta} p_{\theta \theta}^{v}\right)\right]\right\}=0
\end{gathered}
$$

The boundary conditions must allow for the fact that the contribution of the $\Delta f_{p}(\mathbf{x}, \mathbf{G}, v)$ function given by Equation (2.5) to the hydrodynamic values vanishes at an infinite distance from sphere

$$
\left.\Delta f_{p}(\mathbf{x}, \mathbf{G}, v)\right|_{r \rightarrow \infty} \rightarrow 0
$$

At the surface of the sphere, i.e., at $r=a$, one has to impose the no-leak and no-slip conditions

$$
\left.n U_{r}\right|_{r=a}=\left.0 \quad n U_{\theta}\right|_{r=a}=0
$$

Heat flux $q_{r}$ traversing each element of the sphere surface must be counterbalanced by the heat flux within the sphere $q_{r}^{\text {cond }}$ and the thermal radiation $\Delta E$

$$
\left.q_{r}\right|_{r=a}=\left.\left(q_{r}^{\text {cond }}+\Delta E\right)\right|_{r=a}
$$




$$
q_{r}^{\text {cond }}=-\gamma \frac{\partial T}{\partial r} \quad \Delta E=\sigma\left(T-T_{0}\right)
$$

Here, $\gamma$ is the coefficient of the thermal conductivity of the sphere, and $\sigma$ is the coefficient of the sphere emissivity.

\section{Flow around a Sphere at Small Re}

Let us formulate dimensionless parameters $\mathrm{Ma}^{2}=m U_{0}^{2} / k T_{0}$, where Ma denotes the Mach number, and the Reynolds number $\operatorname{Re}=2 m n_{0} U_{0} a / \tilde{\eta}_{0}, \tilde{\eta}_{0}=\tilde{\eta}\left(T_{0}\right)$. Multimoment hydrodynamics Equations (2.9)-(2.13) will be brought to a dimensionless form in a conventional manner [7]. It turns out that the dimensionless equations contain $\mathrm{Ma}^{2}$ and $\mathrm{Re}$. Hydrodynamic values can be represented as parametric series

$$
F=F_{0} \sum_{k=0}^{\infty} \sum_{l=-1}^{\infty}\left(\mathrm{Ma}^{2}\right)^{k}(\mathrm{Re})^{l} \hat{F}^{(k, l)}=F_{0} \sum_{k=0}^{\infty} \sum_{l=-1}^{\infty} \widehat{F}^{(k, l)}
$$

Here, $F=F(\mathbf{x})$ sequentially assumes values $n(\mathbf{x}) ; n(\mathbf{x}) \mathbf{U}(\mathbf{x}) ; p^{v}(\mathbf{x}), s^{G}(\mathbf{x})$, and $s_{i j}^{G}(\mathbf{x}) ; \mathbf{Q}^{G}(\mathbf{x})$ and $\mathbf{Q}^{v}(\mathbf{x})$, and $F_{0}$ sequentially assumes values $n_{0} ; n_{0} U_{0} ; n_{0} k T_{0} ; n_{0} k T_{0} U_{0}$. Symbols ${ }^{\wedge}$ and appear over dimensionless quantities. Multimoment hydrodynamics Equations (2.12), (2.13) are written with the Navier-Stokes accuracy, by which token expansion (3.1) contains no terms with $l \leq-2$.

Consider the flow regime at small Reynolds number $\mathrm{Re} \ll 1$. At the hydrodynamic stage of description characterized by small values of the Knudsen number $\mathrm{Kn} \ll 1$, Ma $\sim \mathrm{Kn} \cdot \mathrm{Re} \ll 1$. Terms of the order of $n_{0} k T_{0} \mathrm{Ma}^{2} / \mathrm{Re}$ dominate expansion (3.1) of the second-order moments $p^{v}$ and $S_{i j}^{G}(\mathbf{x})$ in the flow regime in question. In expansions (3.1) of hydrodynamic values $n, p^{v}$, and $S_{i j}^{G}$, it will suffice to retain only $\widehat{n}^{(1,-1)}, \widehat{p}^{v(1,-1)}$, and $\widehat{S}_{i j}^{G(1,-1)}$, respectively. Expansion (3.1) of the particle density flux $n \mathbf{U}$ is reasonably be restricted to $(\overparen{n \mathbf{U}})^{(0,0)}$, which provides for the $n_{0} k T_{0} \mathrm{Ma}^{2} / \mathrm{Re}$ desired order of the terms in Equation (2.7) for $p_{i j}^{v}$. As noted in [6], the Navier-Stokes approximation is not accurate enough to calculate temperature components $\widehat{T}^{G(1,-1)}$ and $\widehat{T}^{v(1,-1)}$. Thus, at $\operatorname{Re} \ll 1$, the spatial dependence of temperature is neglected.

Let us truncate expansion (2.5) to the three lowest-order terms

$$
\begin{gathered}
\Delta f_{p}^{(0)}(\mathbf{x}, \mathbf{G}, v)=4 \pi\left(\frac{M}{2 \pi k T_{0}}\right)^{3 / 2}\left(\frac{\mu}{2 \pi k T_{0}}\right)^{3 / 2} \exp \left[-\frac{M \mathbf{G}^{2}}{2 k T_{0}}-\frac{\mu v^{2}}{2 k T_{0}}\right]\left\{c_{1} G_{z} \Phi_{r}^{-1}+c_{2} \Phi_{z}+c_{3} G_{z} \Phi_{r}^{2}\right\} \\
c_{1}=c_{1-100} \quad c_{2}=c_{0010} \quad c_{3}=c_{1200}
\end{gathered}
$$

Each of three terms of series (3.2) can contribute to each principal hydrodynamic value $\left(n, n \mathbf{U}, p^{v}, s^{G}\right.$, $s_{i j}^{G}, \mathbf{q}^{v}$, and $\mathbf{q}^{G}$ ). Considering that the principal hydrodynamic values are linearly independent, coefficients $c_{i}, i=1,2,3$, of expansion (3.2) can be written as linear combinations of six arbitrarily chosen components

$$
c_{i}=c_{i}^{(k, l)}(\mathbf{G}, v)=\sum_{r, s}\left(\frac{M G^{2}}{2 k T_{0}}\right)^{r}\left(\frac{\mu v^{2}}{2 k T_{0}}\right)^{s} c_{i(r, s)}^{(k, l)}
$$

To calculate the hydrodynamic values (2.6), the first component of series (3.2) has to be integrated with respect to $\mathbf{G}$ with the appropriate weight function of velocities $\mathbf{G}$ and $v$ within region $W_{2}$, and the second and third components, within region $W_{1}$. Why $\Delta f_{p}^{(0)}$ is approximated by Equation (3.2), and its components are integrated in such a way is explained below. The integration yields

$$
\begin{gathered}
n U_{r}=n_{0} U_{0} \cos \theta+2 A_{1} \cos \theta \frac{a}{r}+2 A_{2} \cos \theta \frac{a^{3}}{r^{3}} \quad A_{1}=n_{0} U_{0} \hat{A}_{1}^{(0,0)} \\
n U_{\theta}=-n_{0} U_{0} \sin \theta-A_{1} \sin \theta \frac{a}{r}+A_{2} \sin \theta \frac{a^{3}}{r^{3}} \quad A_{2}=n_{0} U_{0} \hat{A}_{2}^{(0,0)} \\
n=n_{0}+2 A_{3} \cos \theta \frac{a^{2}}{r^{2}} \quad A_{3}=n_{0}\left(\frac{\mathrm{Ma}^{2}}{\mathrm{Re}}\right) \hat{A}_{3}^{(1,-1)}
\end{gathered}
$$




$$
\begin{gathered}
p^{v}=n_{0} k T_{0}+2 D_{3} k T_{0} \cos \theta \frac{a^{2}}{r^{2}} \quad D_{3} k T_{0}=n_{0} k T_{0}\left(\frac{\mathrm{Ma}^{2}}{\mathrm{Re}}\right) \hat{D}_{3}^{(1,-1)} \\
s^{G}=n_{0} k T_{0}+4 B_{3} k T_{0} \cos \theta \frac{a^{2}}{r^{2}} \quad B_{3} k T_{0}=n_{0} k T_{0}\left(\frac{\mathrm{Ma}^{2}}{\mathrm{Re}}\right) \hat{B}_{3}^{(1,-1)} \\
s_{r r}^{G}=8 B_{3} k T_{0} \cos \theta\left(\frac{a^{2}}{r^{2}}-\frac{a^{4}}{r^{4}}\right) \quad s_{\theta \theta}^{G}=s_{\varphi \varphi}^{G}=-4 B_{3} k T_{0} \cos \theta\left(\frac{a^{2}}{r^{2}}-\frac{a^{4}}{r^{4}}\right) \quad s_{r \theta}^{G}=-4 B_{3} k T_{0} \sin \theta \frac{a^{4}}{r^{4}}
\end{gathered}
$$

Here,

$$
\begin{gathered}
A_{1}=\frac{\pi}{2 a}\left(\frac{2 k T_{0}}{\pi M}\right)^{1 / 2}\left(c_{1(0,0)}^{(0,0)}+2 c_{1(1,0)}^{(0,0)}+\frac{3}{2} c_{1(0,1)}^{(0,0)}\right) \\
A_{2}=\frac{\pi a}{2}\left(\frac{2 k T_{0}}{\pi M}\right)^{3 / 2}\left(c_{2(0,0)}^{(0,0)}+3 c_{2(1,0)}^{(0,0)}+\frac{3}{2} c_{2(0,1)}^{(0,0)}+12 c_{2(2,0)}^{(0,0)}+60 c_{2(3,0)}^{(0,0)}+\frac{9}{2} c_{2(1,1)}^{(0,0)}\right) \\
A_{3}=\frac{\pi a^{2}}{2}\left(\frac{2 k T_{0}}{\pi M}\right)^{3 / 2}\left(c_{3(0,0)}^{(1,-1)}+3 c_{3(1,0)}^{(1,-1)}+\frac{3}{2} c_{3(0,1)}^{(1,-1)}+12 c_{3(2,0)}^{(1,-1)}+60 c_{3(3,0)}^{(1,-1)}+\frac{9}{2} c_{3(1,1)}^{(1,-1)}\right) \\
B_{3}=\frac{\pi a^{2}}{2}\left(\frac{2 k T_{0}}{\pi M}\right)^{3 / 2}\left(c_{3(0,0)}^{(1,-1)}+4 c_{3(1,0)}^{(1,-1)}+\frac{3}{2} c_{3(0,1)}^{(1,-1)}+20 c_{3(2,0)}^{(1,-1)}+120 c_{3(3,0)}^{(1,-1)}+6 c_{3(1,1)}^{(1,-1)}\right) \\
D_{3}=\frac{\pi a^{2}}{2}\left(\frac{2 k T_{0}}{\pi M}\right)^{3 / 2}\left(c_{3(0,0)}^{(1,-1)}+3 c_{3(1,0)}^{(1,-1)}+\frac{5}{2} c_{3(0,1)}^{(1,-1)}+12 c_{3(2,0)}^{(1,-1)}+60 c_{3(3,0)}^{(1,-1)}+\frac{15}{2} c_{3(1,1)}^{(1,-1)}\right) \\
c_{1(r, s)}^{(0,0)}=a\left(\frac{M}{k T_{0}}\right)^{1 / 2} n_{0} U_{0} \hat{c}_{1(r, s)}^{(0,0)} c_{2(r, s)}^{(0,0)}=\frac{1}{a}\left(\frac{M}{k T_{0}}\right)^{3 / 2} n_{0} U_{0} \hat{c}_{2(r, s)}^{(0,0)} c_{3(r, s)}^{(1,-1)}=\frac{2}{a^{2}}\left(\frac{M}{k T_{0}}\right)^{3 / 2} n_{0}\left(\frac{\mathrm{Ma}^{2}}{\operatorname{Re}}\right) \hat{c}_{3(r, s)}^{(1,-1)}
\end{gathered}
$$

The order of coefficients $A_{1}$ and $A_{2}$ appearing in Equations $(3.4,3.5)$ depends on the only density flux component, $(\overparen{n \mathbf{U}})^{(0,0)}$, retained in expansion (3.1) at $\mathrm{Re} \ll 1$. The order of coefficients $A_{3}, B_{3}$, and $D_{3}$ depends on components $\widehat{n}^{(1,-1)}, \hat{p}^{v(1,-1)}$, and $\widehat{S}_{i j}^{G(1,-1)}$ of expansion (3.1). Using one of six linear combinations of coefficients $c_{2(r, s)}^{(0,0)}$, we eliminate the contribution to the particle density $n$, proportional to $n_{0}$ Ma, which is missed in expansion (3.1). Using two linear combinations of coefficients $c_{2(r, s)}^{(0,0)}$, we eliminate the contribution to $p^{v}$ and $s^{G}$ that is proportional to $n_{0} k T_{0} \mathrm{Ma}$ and is missed in expansion (3.1). Two other linear combinations of $c_{2(r, s)}^{(0,0)}$ enable us to eliminate the contribution to $\mathbf{q}^{v}$ and $\mathbf{q}^{G}$ that is proportional to $n_{0} k T_{0} U_{0}$. The reason why $\mathbf{q}^{v}$ and $\mathbf{q}^{G}$ contain no terms proportional to $n_{0} k T_{0} U_{0}$ will be elucidated in Section 4. The last, sixth linear combination of $c_{2(r, s)}^{(0,0)}$ yields $A_{2}$ in Equation (3.5). Three linear combinations of $c_{3(r, s)}^{(1,-1)}$ enable us to eliminate the contribution to $n \mathbf{U}$ being proportional to $n_{0} U_{0} \mathrm{Ma} / \mathrm{Re}$ and the contribution to $\mathbf{q}^{v}$ and $\mathbf{q}^{G}$ being proportional to $n_{0} U_{0} k T_{0} \mathrm{Ma} / \mathrm{Re}$, which are missed in Equation (3.1). The first term of series (3.2) contributes only to the first and the third moments of the $f_{p}(\mathbf{x}, \mathbf{G}, v)$ function. Two linear combinations of $c_{1(r, s)}^{(0,0)}$ offer means of eliminating the contribution to $\mathbf{q}^{v}$ and $\mathbf{q}^{G}$ being proportional to $n_{0} k T_{0} U_{0}$.

Using boundary conditions (2.15) we obtain $\hat{A}_{1}^{(0,0)}=-3 / 4, \hat{A}_{2}^{(0,0)}=1 / 4$. The components of tensor $p_{i j}^{v}(\mathbf{x})$ (2.7) can be determined from Equation (3.4). Substituting $p_{i j}^{v}(\mathbf{x})$ and $p^{v}(\mathbf{x})$ from (3.4) into any of the equations of momentum conservation (2.12), we obtain $\hat{D}_{3}^{(1,-1)}=-3 / 2$. When analyzing the multimoment hydrodynamics equations in the order of $n_{0} k T_{0} \mathrm{Ma}^{2} / \mathrm{Re}$ [6], it was inferred that $\hat{s}_{i j}^{G(1,-1)}=\hat{p}_{i j}^{v(1-1)}=\hat{p}_{i j}^{(1-1)}$, 
$\hat{s}^{G(1-1)}=\hat{p}^{v(1-1)}=\hat{p}^{(1-1)}$, hence, $\hat{B}_{3}^{(1,-1)}=-3 / 4, \quad \hat{A}_{3}^{(1,-1)}=-3 / 2$. Substituting the foregoing expressions for the coefficients $\hat{A}_{1}^{(0,0)}, \hat{A}_{2}^{(0,0)}, \hat{A}_{3}^{(1,-1)}, \hat{B}_{3}^{(1,-1)}, \hat{D}_{3}^{(1,-1)}$ into Equation (3.4) and using definitions (2.8), we obtain

$$
\begin{gathered}
n_{0} U_{0}\left(\overparen{n U_{r}}\right)^{(0,0)}=n_{0} U_{0} \cos \theta\left(1-\frac{3 a}{2 r}+\frac{a^{3}}{2 r^{3}}\right) \quad n_{0} U_{0}\left(\overparen{n U_{\theta}}\right)^{(0,0)}=n_{0} U_{0} \sin \theta\left(-1+\frac{3 a}{4 r}+\frac{a^{3}}{4 r^{3}}\right) \\
n_{0} \widehat{n}^{(1,-1)}=-\frac{3 \tilde{\eta}_{0} U_{0}}{2 a k T_{0}} \cos \theta \frac{a^{2}}{r^{2}} \quad n=n_{0}\left(1+\widehat{n}^{(1,-1)}\right) \\
n_{0} k T_{0} \widehat{p}^{(1,-1)}=-\frac{3 \tilde{\eta}_{0} U_{0}}{2 a} \cos \theta \frac{a^{2}}{r^{2}} \quad p=n_{0} k T_{0}\left(1+\widehat{p}^{(1,-1)}\right) \\
n_{0} k T_{0} \hat{p}_{r r}^{(1,-1)}=-\frac{3 \tilde{\eta}_{0} U_{0}}{a} \cos \theta\left(\frac{a^{2}}{r^{2}}-\frac{a^{4}}{r^{4}}\right) \quad n_{0} k T_{0} \widehat{p}_{r \theta}^{(1,-1)}=\frac{3 \tilde{\eta}_{0} U_{0}}{2 a} \sin \theta \frac{a^{4}}{r^{4}} \\
n_{0} k T_{0} \hat{p}_{\theta \theta}^{(1,-1)}=n_{0} k T_{0} \hat{p}_{\varphi \varphi}^{(1,-1)}=\frac{3 \tilde{\eta}_{0} U_{0}}{2 a} \cos \theta\left(\frac{a^{2}}{r^{2}}-\frac{a^{4}}{r^{4}}\right) \quad p_{i j}=n_{0} k T_{0} \hat{p}_{i j}^{(1-1)}
\end{gathered}
$$

Distributions (3.6), (3.7) follow from the classic hydrodynamics equations [7] for the Stokes flow, which are identical to the multimoment hydrodynamics equations in the limit $\mathrm{Re} \ll 1$ [6].

The hydrodynamic values (3.6), (3.7) assume the form of a linear combination of the products of $(a / r)^{k}$ by trigonometric functions. To calculate (3.6), (3.7), the first component of Equation (3.2) was integrated with respect to $\mathbf{G}$ within the $W_{2}$ region, and the second and third components, within the $W_{1}$ region. If, on the contrary, the first component is integrated within $W_{1}$, and the others, within $W_{2}$, Equation (3.4) contain the products of irrational $\left(1-a^{2} / r^{2}\right)^{k+1 / 2}$ and trigonometric functions. However, with such products in Equation (3.4), it is impossible to satisfy boundary conditions (2.15) and equations of momentum conservation (2.12) simultaneously. When the higher-order terms are being retained in expansion (2.5), the functions will appear in Equation (3.4) which are nonlinear in $\cos \theta$ and $\sin \theta$ and the Equations (2.15) and (2.12) cannot, as previously, be satisfied simultaneously.

\section{Stationary Flow around a Sphere at Moderately High Re}

Consider a flow at a moderately high $\mathrm{Re}$ and $\mathrm{Ma}^{2} \ll 1$. In this case, $(\overparen{n \mathbf{U}})^{(0,0)}$ will be retained together with two other components of expansion (3.1) of the flow density $n \mathbf{U}:(\overparen{n \mathbf{U}})^{(0, l+1)}$ and $(\overparen{n \mathbf{U}})^{(1, l)}, l=0,1, \cdots$. In addition to already obtained components of expansions (3.1) of the zero- and second-order moments of the $f_{p}(\mathbf{x}, \mathbf{G}, v)$ pair distribution function, $n^{(k, l)}, p^{v(k, l)}$, and $S_{i j}^{G(k, l)}$ with $k=0,1$ и $l=0,1, \cdots$, will be taken into account. Expansions (3.1) of the energy fluxes $\mathbf{Q}^{G}$ and $\mathbf{Q}^{v}$ will be limited to $\mathbf{Q}^{G(k, l)}$ and $\mathbf{Q}^{v(k, l)}$ with $k=0,1$ и $l=-1,0, \cdots$.

Expressions for hydrodynamic values (2.6) will be constructed by the same principle as in Section 3-as linear combinations of the products of $(a / r)^{k}$ and trigonometric functions. This principle governs both the structure of the retained terms of expansion (2.5) and the region of integration with respect to $\mathbf{G}$ for each term of expansion (2.5). In addition to three terms given by Equation (3.2), the following components of expansion (2.5) will be included

$$
\begin{gathered}
\Delta f_{p}^{(1)}(\mathbf{x}, \mathbf{G}, v)=\Delta f_{p}^{(1,0)}(\mathbf{x}, \mathbf{G}, v)+\Delta f_{p}^{(1,1)}(\mathbf{x}, \mathbf{G}, v) \\
\Delta f_{p}^{(1,0)}(\mathbf{x}, \mathbf{G}, v)=4 \pi\left(\frac{M}{2 \pi k T_{0}}\right)^{3 / 2}\left(\frac{\mu}{2 \pi k T_{0}}\right)^{3 / 2} \exp \left[-\frac{M \mathbf{G}^{2}}{2 k T_{0}}-\frac{\mu v^{2}}{2 k T_{0}}\right] \\
\times\left\{c_{4} \Phi_{r}^{-1}+c_{5} G_{z}^{2} \Phi_{r}^{-1}+c_{6} \Phi_{r}^{-3} F_{x y}^{2}+c_{7} G_{z}+c_{8} G_{z} \Phi_{r}^{-1}+c_{9} G_{z} \Phi_{z}+c_{10} G_{z} \Phi_{r}^{-1} \Phi_{z}\right\} \\
c_{4}=c_{0-100} \quad c_{5}=c_{2-100} \quad c_{6}=c_{0-302} \quad c_{7}=c_{1000} \quad c_{8}=c_{1-100} \quad c_{9}=c_{1010} \quad c_{10}=c_{1-110}
\end{gathered}
$$




$$
\begin{aligned}
& \Delta f_{p}^{(1,1)}(\mathbf{x}, \mathbf{G}, v)= 4 \pi\left(\frac{M}{2 \pi k T_{0}}\right)^{3 / 2}\left(\frac{\mu}{2 \pi k T_{0}}\right)^{3 / 2} \exp \left[-\frac{M G^{2}}{2 k T_{0}}-\frac{\mu v^{2}}{2 k T_{0}}\right] \\
& \times\left\{c_{11} G_{z} \Phi_{r}^{-1}+c_{12} G_{z}^{3} \Phi_{r}^{-1}+c_{13} \Phi_{z}+c_{14} G_{z}^{2} \Phi_{z}+c_{15} G_{z}^{2} \Phi_{r}^{-1} \Phi_{z}\right. \\
&\left.+c_{16}+c_{17} G_{z}^{2}+c_{18} G_{z}^{2} \Phi_{r}^{-1}+c_{19} G_{z}^{4}+c_{20} G_{z}^{4} \Phi_{r}^{-1}+c_{21} G_{z}^{4} \Phi_{r}\right\} \\
& c_{11}=c_{1-100} \quad c_{12}= c_{3-100} \quad c_{13}=c_{0010} \quad c_{14}=c_{2010} \quad c_{15}=c_{2-110} \quad c_{16}=c_{0000} \\
& c_{17}=c_{2000} \quad c_{18}=c_{2-100} \quad c_{19}=c_{4000} \quad c_{20}=c_{4-100} \quad c_{21}=c_{4100}
\end{aligned}
$$

Generally, each component of expansions $(4.1,4.2)$ contributes to each principle hydrodynamic value, irrespective of the order of terms retained in expansion (3.1). Considering that the principle hydrodynamic values are linearly independent, each coefficient $c_{i}, i=4, \cdots, 21$, should be represented as series (3.3) of six terms for any considered order of expansion (3.1). Function $\Delta f_{p}^{(1,0)}$ given by (4.1) is used to calculate the zero- and the second-order moments. The contribution of $\Delta f_{p}^{(1,0)}$ is eliminated from the first- and third-order moments through the use of Equation (3.3), as in Section 3. Function $\Delta f_{p}^{(1,1)}$ (4.2) is used exclusively to calculate the first- and the third-order moments. The terms of Equation (4.1) proportional to $c_{4}, c_{5}, c_{6}$ and the terms of Equation (4.2) proportional to $c_{11}, c_{12}$ are integrated with respect to $\mathbf{G}$ within region $W_{2}$. The terms of Equation (4.1) proportional to $c_{7}, \cdots, c_{10}$ and the terms of Equation (4.2) proportional to $c_{13}, \cdots, c_{21}$ are integrated with respect to $\mathbf{G}$ within region $W_{1}$.

Distributions of the principle hydrodynamics values (2.6) are given in [8] by Equations (A.1)-(A.7). The expressions (A.1)-(A.7) are written in terms of coefficients $C_{i}, i=1, \cdots, 50$. Coefficients $C_{i}$ are expressed in terms of $c_{i}, i=4, \cdots, 21$ which appear in Equations (4.1), (4.2). Each coefficient $c_{i}$ is expanded into series (3.3) of six $r, s$-terms for each considered $k, l$-order of expansion (3.1). In what follows, the relationships between $C_{i}$ and $c_{i}$ required to derive explicit expressions for $\Delta f_{p}^{(1,0)}$ and $\Delta f_{p}^{(1,1)}$, will be omitted. Each coefficient $C_{i}, i=1, \cdots, 50$, contains dimensionless multiplier $\hat{C}_{i}$.

In order to calculate $\hat{C}_{i}, i=1, \cdots, 50$, one needs, as in Section 3, to satisfy boundary conditions (2.14)-(2.16) and equations of conservation (2.12), (2.13). Imposing boundary conditions (2.15) upon the particle density flux $n \mathbf{U}$ (A.5) from [8], we express 16 coefficients $\hat{C}_{i}, i=34, \cdots, 44,46, \cdots, 50$ in terms of 34 remained coefficients [8]. As revealed in [6], the $n_{0} k T_{0} U_{0} \mathrm{Ma}^{2} \mathrm{Re}^{l-1}$-order approximation to the total energy-flux vector $\mathbf{Q}$ $(l=0,1, \cdots)$ is

$$
Q_{i}=-\tilde{\lambda}\left[\frac{\partial T^{*}}{\partial x_{i}}+\frac{1}{5 n k} \frac{\partial s_{i j}^{G}}{\partial x_{j}}\right]+Q_{i}^{0} \quad T^{*}=\frac{s^{G}+p^{v}}{2 n} \quad Q_{i}^{0}=\frac{m n_{0} U_{i 0} U_{0}^{2}}{2}
$$

Here $\tilde{\lambda}=15 k \tilde{\eta} / 4 m$ is the coefficient of the thermal conductivity. Substituting the expressions for hydrodynamic values (A.1)-(A.4) from [8] into Equation (4.3), it is possible to express 8 coefficients $\hat{C}_{i}, i=26, \cdots, 33$, in terms of 26 remained coefficients. Substitute Equations (A.2), (A.5), and (A.6) from [8] into equation of momentum conservation (2.12) and equate the coefficients of the $(a / r)^{k} \cos ^{n} \theta \sin ^{m} \theta$ products for either of the orders, $n_{0} k T_{0} \mathrm{Ma}^{2} / a$ and $n_{0} k T_{0} \mathrm{Ma}^{4} / a \mathrm{Re}$. Then 6 coefficients $\hat{C}_{i}, i=21, \cdots, 25,45$, can be expressed in terms of 20 remained coefficients $\hat{C}_{i}, \quad i=1, \cdots, 20$, [8]. Eventually, the expressions for the principle hydrodynamic values (2.6) can be written as

$$
\begin{gathered}
n=n_{0}\left(1+\widehat{n}^{(1,-1)}+\widehat{n}^{(1)}\right) \quad \hat{n}^{(1)}=\sum_{l=0}^{\infty} \hat{n}^{(1, l)} \\
\widehat{n}^{(1)}=\mathrm{Ma}^{2}\left[-(1 / 2) \hat{C}_{1}\left(\cos ^{2} \theta+1\right)(a / r)-\hat{C}_{2}\left(3 \cos ^{2} \theta-1\right)(a / r)^{3}-2 \hat{C}_{3}(a / r)-\hat{C}_{4} \cos \theta(a / r)^{2}\right] \\
p^{v}=n_{0} k T_{0}\left(1+\hat{p}^{v(1,-1)}+\hat{p}^{v(1)}\right) \quad \hat{p}^{v(1)}=\sum_{l=0}^{\infty} \hat{p}^{v(1, l)} \\
\hat{p}^{v(1)}=\mathrm{Ma}^{2}\left[-40 \hat{C}_{20}\left(3 \cos ^{2} \theta-1\right)(a / r)^{3}\right]
\end{gathered}
$$




$$
\begin{aligned}
& s^{G}=n_{0} k T_{0}\left(1+\widehat{s}^{G(1,-1)}+\widehat{s}^{G(1)}\right) \quad \widehat{s}^{G(1)}=\sum_{l=0}^{\infty} \widehat{s}^{G(1, l)} \\
& s^{G(1)}=\mathrm{Ma}^{2}\left[\left(-(5 / 3) \hat{C}_{1}+(2 / 3) \hat{C}_{19}\right)\left(\cos ^{2} \theta+1\right)(a / r)+(8 / 3) \hat{C}_{6}(a / r)\right. \\
& \left.+\left(2 \hat{C}_{5}+40 \hat{C}_{20}\right)\left(3 \cos ^{2} \theta-1\right)(a / r)^{3}+(4 / 3) \hat{C}_{7} \cos \theta(a / r)^{2}\right] \\
& s_{i j}^{G}=n_{0} k T_{0}\left(\widehat{s}_{i j}^{G(1,-1)}+\widehat{s}_{i j}^{G(1)}\right) \quad \widehat{s}_{i j}^{G(1)}=\sum_{l=0}^{\infty} \widehat{s}_{i j}^{G(1, l)} \\
& \widehat{s}_{r r}^{G(1)}=\mathrm{Ma}^{2}\left[\left(-(5 / 12) \hat{C}_{1}+(1 / 6) \hat{C}_{19}\right)\left(11 \cos ^{2} \theta-1\right)(a / r)+(4 / 3) \hat{C}_{6}(a / r)\right. \\
& +\left(4 \hat{C}_{5}+80 \hat{C}_{20}\right)\left(3 \cos ^{2} \theta-1\right)(a / r)^{3}-4 \hat{C}_{8}\left(3 \cos ^{2} \theta-1\right)(a / r)^{5} \\
& \left.+3 \hat{C}_{18}\left(3 \cos ^{2} \theta-1\right)(a / r)+(8 / 3) \hat{C}_{7} \cos \theta(a / r)^{2}-2 \hat{C}_{9} \cos \theta(a / r)^{4}\right] \\
& \widehat{s}_{\theta \theta}^{G(1)}=\mathrm{Ma}^{2}\left[\left(-(5 / 48) \hat{C}_{1}+(1 / 24) \hat{C}_{19}\right)\left(-31 \cos ^{2} \theta+11\right)(a / r)-(2 / 3) \hat{C}_{6}(a / r)\right. \\
& -\left(2 \hat{C}_{5}+40 \hat{C}_{20}\right)\left(3 \cos ^{2} \theta-1\right)(a / r)^{3}+\hat{C}_{8}\left(7 \cos ^{2} \theta-3\right)(a / r)^{5} \\
& \left.+(3 / 4) \hat{C}_{18}\left(-7 \cos ^{2} \theta+3\right)(a / r)-(4 / 3) \hat{C}_{7} \cos \theta(a / r)^{2}+\hat{C}_{9} \cos \theta(a / r)^{4}\right] \\
& \widehat{S}_{\varphi \varphi}^{G(1)}=\operatorname{Ma}^{2}\left[\left(-(5 / 48) \hat{C}_{1}+(1 / 24) \hat{C}_{19}\right)\left(-13 \cos ^{2} \theta-7\right)(a / r)-(2 / 3) \hat{C}_{6}(a / r)-\left(2 \hat{C}_{5}+40 \hat{C}_{20}\right)\left(3 \cos ^{2} \theta-1\right)(a / r)^{3}\right. \\
& \left.+\hat{C}_{8}\left(5 \cos ^{2} \theta-1\right)(a / r)^{5}+(3 / 4) \hat{C}_{18}\left(-5 \cos ^{2} \theta+1\right)(a / r)-(4 / 3) \hat{C}_{7} \cos \theta(a / r)^{2}+\hat{C}_{9} \cos \theta(a / r)^{4}\right] \\
& \widehat{S}_{r \varphi}^{G(1)}=\mathrm{Ma}^{2}\left[\left((5 / 2) \hat{C}_{1}-\hat{C}_{19}\right) \cos \theta \sin \theta(a / r)+\left(6 \hat{C}_{5}+120 \hat{C}_{20}\right) \cos \theta \sin \theta(a / r)^{3}\right. \\
& \left.-8 \hat{C}_{8} \cos \theta \sin \theta(a / r)^{5}-6 \hat{C}_{18} \cos \theta \sin \theta(a / r)-\hat{C}_{9} \sin \theta(a / r)^{4}\right] \\
& n U_{i}=n_{0} U_{0}\left[\left(\overparen{n U_{i}}\right)^{(0,0)}+\overparen{n U_{i}}\right] \quad \overparen{n U_{i}}=\sum_{l=0}^{\infty}\left[\left(\overparen{n U_{i}}\right)^{(1, l)}+\left(\overparen{n U_{i}}\right)^{(0, l+1)}\right] \\
& \overparen{n U_{r}}=\operatorname{Ma}^{2}\left[(1 / 15) \hat{C}_{14}\left(3 \cos \theta-5 \cos ^{3} \theta\right)(a / r)\left(1-(a / r)^{2}\right)^{2}\right] \\
& +\operatorname{Re}\left[\hat{C}_{20}\left(3-30 \cos ^{2} \theta+35 \cos ^{4} \theta\right)(a / r)^{2}\left(1-(a / r)^{2}\right)^{2}\right] \\
& \overparen{n U_{\theta}}=\operatorname{Ma}^{2}\left[(1 / 60) \hat{C}_{14}\left(3 \sin \theta-5 \sin \theta \cos ^{2} \theta\right)(a / r)\left(-1-2(a / r)^{2}+3(a / r)^{4}\right)\right] \\
& +\operatorname{Re}\left[\hat{C}_{20}\left(12 \sin \theta \cos \theta-28 \sin \theta \cos ^{3} \theta\right)(a / r)^{4}\left(1-(a / r)^{2}\right)\right] \\
& Q_{i}^{v}=n_{0} k T_{0} U_{0}\left[\widehat{Q}_{i}^{v(0,0)}+\widehat{Q}_{i}^{v(0)}+\widehat{Q}_{i}^{v(1)}\right] \quad \widehat{Q}_{i}^{v(0)}=\sum_{l=0}^{\infty} \widehat{Q}_{i}^{v(0, l+1)} \widehat{Q}_{i}^{v(1)}=\sum_{l=0}^{\infty} \widehat{Q}_{i}^{v(1, l-1)} \\
& \widehat{Q}_{r}^{v(1)}=\mathrm{Ma}^{2}\left[-\hat{C}_{10}\left(\cos \theta-2 \cos ^{3} \theta\right)(a / r)^{3}+(1 / 3) \hat{C}_{11}\left(3 \cos \theta-5 \cos ^{3} \theta\right)(a / r)^{5}+2 \hat{C}_{12} \cos \theta(a / r)\right. \\
& \left.+2 \hat{C}_{13} \cos \theta(a / r)^{3}+2 \hat{C}_{15}(a / r)^{2}+2 \hat{C}_{16} \cos ^{2} \theta(a / r)^{2}+(1 / 2) \hat{C}_{17}\left(1-3 \cos ^{2} \theta\right)(a / r)^{4}\right] \\
& \widehat{Q}_{\theta}^{v(1)}=\operatorname{Ma}^{2}\left[(1 / 2) \hat{C}_{10} \sin \theta \cos ^{2} \theta(a / r)^{3}+(1 / 4) \hat{C}_{11}\left(\sin \theta-5 \sin \theta \cos ^{2} \theta\right)(a / r)^{5}\right. \\
& \left.-\hat{C}_{12} \sin \theta(a / r)+\hat{C}_{13} \sin (a / r)^{3}-\hat{C}_{17} \sin \theta \cos \theta(a / r)^{4}\right]
\end{aligned}
$$




$$
\begin{aligned}
& \widehat{Q}_{i}^{v(0,0)}=(3 / 2)\left[\left(\overparen{n U_{i}}\right)^{(0,0)}-\left(\overparen{n U_{0 i}}\right)\right] \quad \widehat{Q}_{i}^{v(0, l+1)}=3 / 2\left(\overparen{n U_{i}}\right)^{(0, l+1)} \quad l=0,1, \cdots \\
& Q_{i}^{G}=n_{0} k T_{0} U_{0}\left[\widehat{Q}_{i}^{G(0,0)}+\widehat{Q}_{i}^{G(0)}+\widehat{Q}_{i}^{G(1)}\right] \quad \widehat{Q}_{i}^{G(0)}=\sum_{l=0}^{\infty} \widehat{Q}_{i}^{G(0, l+1)} \quad \widehat{Q}_{i}^{G(1)}=\sum_{l=0}^{\infty} \widehat{Q}_{i}^{G(1, l-1)} \\
& \widehat{Q}_{r}^{G(1)}=\mathrm{Ma}^{2}\left[(5 / 3) \hat{C}_{10}\left(\cos \theta-2 \cos ^{3} \theta\right)(a / r)^{3}-(5 / 9) \hat{C}_{11}\left(3 \cos \theta-5 \cos ^{3} \theta\right)(a / r)^{5}\right. \\
& -(10 / 3) \hat{C}_{12} \cos \theta(a / r)-(10 / 3) \hat{C}_{13} \cos \theta(a / r)^{3}-(10 / 3) \hat{C}_{15}(a / r)^{2} \\
& \left.-(10 / 3) \hat{C}_{16} \cos ^{2} \theta(a / r)^{2}-(5 / 6) \hat{C}_{17}\left(1-3 \cos ^{2} \theta\right)(a / r)^{4}\right] \\
& +\mathrm{Ma}^{2} / \operatorname{Re}\left[\left(8 \hat{C}_{7}+20 \hat{C}_{4}\right) \cos \theta(a / r)^{3}+\left(2 \hat{C}_{19}+8 \hat{C}_{6}+20 \hat{C}_{3}\right)(a / r)^{2}\right. \\
& \left.+2 \hat{C}_{19} \cos ^{2} \theta(a / r)^{2}+\left(-18 \hat{C}_{5}+240 \hat{C}_{20}-30 \hat{C}_{2}\right)\left(1-3 \cos ^{2} \theta\right)(a / r)^{4}\right] \\
& \widehat{Q}_{\theta}^{G(1)}=\mathrm{Ma}^{2}\left[-(5 / 6) \hat{C}_{10} \sin \cos ^{2} \theta(a / r)^{3}-(5 / 12) \hat{C}_{11} \sin \theta\left(1-5 \cos ^{2} \theta\right)(a / r)^{5}\right. \\
& \left.+(5 / 3) \hat{C}_{12} \sin \theta(a / r)-(5 / 3) \hat{C}_{13} \sin (a / r)^{3}+(5 / 3) \hat{C}_{17} \sin \theta \cos \theta(a / r)^{4}\right] \\
& +\mathrm{Ma}^{2} / \operatorname{Re}\left[\left(4 \hat{C}_{7}+10 \hat{C}_{4}\right) \sin \theta(a / r)^{3}-\left(-36 \hat{C}_{5}+480 \hat{C}_{20}-60 \hat{C}_{2}\right) \sin \theta \cos \theta(a / r)^{4}\right] \\
& \widehat{Q}_{i}^{G(0,0)}=(5 / 2)\left[\left(\overparen{n U_{i}}\right)^{(0,0)}-\left(\overparen{n U_{0 i}}\right)\right] \quad \widehat{Q}_{i}^{G(0, l+1)}=5 / 2\left(\overparen{n U_{i}}\right)^{(0, l+1)} \quad l=0,1, \cdots
\end{aligned}
$$

The terms proportional to $n_{0} \operatorname{Re}^{l}$ and $n_{0} k T_{0} \operatorname{Re}^{l}, l=1,2, \cdots$, were dropped from Equations (4.4)-(4.7) for the zero-and the second-order moments, respectively, because for Equation (2.13) to be satisfied in the order of $n_{0} k T_{0} U_{0} / a$, all the coefficients of these terms must be zero. For Equation (2.13) to be good in the order of $n_{0} k T_{0} U_{0} \mathrm{Ma}^{2} / a \mathrm{Re}$, one has to put expression (4.9) for $\widehat{Q}_{i}^{v(0, l)}, l=0,1, \cdots$ From the conditions for no heat flux $\mathbf{q}$ at the surface of the sphere (at $r=a$ ) and at infinite distance from the sphere the expression follows (4.10) for $\widehat{Q}_{i}^{G(0, l)}, l=0,1, \cdots$ Let us next substitute Equations (4.4)-(4.10), and (3.6), (3.7) into Equation (2.13) and equate the coefficients of $(a / r)^{k} \cos ^{m} \theta$ products of the order of $n_{0} k T_{0} U_{0} \mathrm{Ma}^{2} / a$ Re. It turns out that all but the $(a / r)^{3}$ and $(a / r)^{3} \cos ^{2} \theta$ products satisfy the law of energy conservation. These two products satisfy Equation (2.13) at

$$
\hat{C}_{19}=0
$$

As previously, Equation (2.13) must be satisfied for each $(a / r)^{k} \cos ^{m} \theta$ product of the order of $n_{0} k T_{0} U_{0} \mathrm{Ma}^{4} / a \mathrm{Re}$ individually. Eventually we obtained a nonlinear set of eighteen algebraic equations (A.8-A.10) from [8]. In [8], of eighteen equations we retained sixteen ones.

The total energy flux equals the heat flux at the surface of the sphere, $\left.\mathbf{Q}\right|_{r=a}=\left.\mathbf{q}\right|_{r=a}$. Using the solutions of the internal boundary problem for the Laplace equation, the temperature distribution inside the sphere can be expanded in terms of Legendre polynomials [9]. The coefficients of this expansion can be calculated by matching the temperature distribution $k T=p / n$ (4.4)-(4.7) at the surface of the sphere with the distribution derived from the solution of the internal boundary problem. The resulting balance of heat fluxes at the surface of the sphere (2.16) in order of $n_{0} k T_{0} U_{0} \mathrm{Ma}^{2} / \mathrm{Re}$ becomes

$$
\begin{gathered}
2 \hat{C}_{3}+(4 / 5) \hat{C}_{6}+(4 / 15) \hat{C}_{19}=4 \hat{\sigma}\left[2 \hat{C}_{3}+(4 / 3) \hat{C}_{6}-(4 / 9) \hat{C}_{1}+(4 / 9) \hat{C}_{19}+1 / 3\right] \\
2 \hat{C}_{4}+(4 / 5) \hat{C}_{7}+(1 / 15) \operatorname{Re}^{-1}=(4 \hat{\sigma}-\hat{\gamma})\left[\hat{C}_{4}+(2 / 3) \hat{C}_{7}\right]
\end{gathered}
$$




$$
6 \hat{C}_{2}+(18 / 5) \hat{C}_{5}-48 \hat{C}_{20}+(2 / 15) \hat{C}_{19}=(4 \hat{\sigma}-2 \hat{\gamma})\left[2 \hat{C}_{2}+2 \hat{C}_{5}-(2 / 9) \hat{C}_{1}+(2 / 9) \hat{C}_{19}\right]
$$

Here, $\hat{\gamma}=\left.(\tilde{\gamma} / \tilde{\lambda})\right|_{T_{0}}, \hat{\sigma}=\left.\left(\tilde{\sigma} T^{3} a / \tilde{\lambda}\right)\right|_{T_{0}}$.

Sixteen equations (П.8, П.9, П.13) from [8] supplemented with three Equation (4.12), and Equation (4.11) form closed nonlinear set of twenty algebraic equations for twenty coefficients $\hat{C}_{i}, i=1, \cdots, 20$. Four coefficients, namely $\hat{C}_{1}, \hat{C}_{2}, \hat{C}_{3}$, and $\hat{C}_{4}$, appear in the distribution of particle density (4.4). Seven coefficients $\hat{C}_{5}$, $\hat{C}_{6}, \hat{C}_{7}, \hat{C}_{8}, \hat{C}_{9}, \hat{C}_{18}$, and $\hat{C}_{19}$, are responsible for the distributions of pressure and stress (4.6), (4.7). Seven coefficients, $\hat{C}_{10}, \hat{C}_{11}, \hat{C}_{12}, \hat{C}_{13}, \hat{C}_{15}, \hat{C}_{16}$, and $\hat{C}_{17}$, appear in the distributions of energy flux (4.9), (4.10), two coefficients, $\hat{C}_{14}$ and $\hat{C}_{20}$, govern the distribution of particle-density flux (4.8).

When constructing the distributions of hydrodynamic values in Section 3, the series (2.5) was truncated to the terms that contribute linearly in $\cos \theta$ and $\sin \theta$ to these distributions. Going beyond the limits of the $\operatorname{Re} \ll 1$ case, we retained seven terms in Equation (4.1) and eleven terms in Equation (4.2). The expansion (4.1) is used to calculate the zero- and the second-order moments and makes the contributions to Equations (4.4)-(4.7) that are proportional to $1, \cos \theta$, and $\cos ^{2} \theta$. The expansion (4.2) is used to calculate the first- and the thirdorder moments and gives rise to the $r$-components of Equations (4.8)-(4.10) proportional to $\cos \theta$ and $\cos ^{3} \theta$. With higher-order terms of expansions (4.1), (4.2) taken into account, the contributions to Equations (4.4)-(4.10) are proportional to more high powers of $\cos \theta$. We were compelled to make one exception associated with retention of the trajectory invariants proportional to $c_{19}, c_{20}$, and $c_{21}$ in Equation (4.2). Owing to these invariants, the $n U_{r}$ distribution (4.8) involves the component proportional to $\cos ^{4} \theta$, lacking in the expansions of the other hydrodynamic values (2.6). The terms of expansion (4.2) proportional to $c_{19}, c_{20}$, and $c_{21}$ enabled us to allow for the terms of the order of $n_{0} U_{0} \operatorname{Re}^{l}, l=1,2, \cdots$, in the distribution of particle-density flux (4.8). The terms of this order dominate $n \mathbf{U}$ at $\operatorname{Re} \gg 1$.

Numerical integration of the nonlinear set of 20 algebraic equations was carried out at $\hat{\gamma}=100$, and $\hat{\sigma}=0.5$. Calculations have revealed a great many roots. However, at $10<\operatorname{Re} \leq 129.1$, the set has only one invariably stable root $\hat{C}_{i}=\bar{C}_{i}^{(0)}, i=1, \cdots, 20$, displayed in Figure 4 in [8]. According to the $\bar{C}_{i}^{(0)}, i=1, \cdots, 20$, solution, an axisymmetric recirculating zone is formed in the wake behind the sphere at $\operatorname{Re} 20$. This recirculating zone has the shape of an axisymmetric toroidal ring. It expands as Re grows but its shape remains unchanged (Figure 3(a)). At $\operatorname{Re}=\operatorname{Re}_{0}^{*}=129.1$, the system becomes unstable. At $\operatorname{Re} \simeq 1$, the Barnett corrections [7] are known to be commensurate with the Navier-Stokes terms. For this reason the calculations [8] were interrupted at $\mathrm{Re}=10$.

\section{First Unstable Flow Regime}

As the flow around sphere becomes unstable, the problem becomes nonstationary. As in the stationary case, the pair distribution function $f_{p}(t, \mathbf{x}, \mathbf{G}, v)$ is represented as

$$
f_{p}(t, \mathbf{x}, \mathbf{G}, v)=f_{p}^{(0)}(\mathbf{G}, v)+\Delta f_{p}(t, \mathbf{x}, \mathbf{G}, v)
$$

Here, $f_{p}^{(0)}(\mathbf{G}, v)$ is given by Equation (2.2), and $\Delta f_{p}(t, \mathbf{x}, \mathbf{G}, v)$ is given by Equation (2.5) with time-dependent coefficients $c_{k l m n}$. In going to a stationary flow, pair distribution functions lose their main property (17) from [3]. Retaining, as previously, 21 trajectory invariants in expansion (2.5), we arrive at distributions (A.1)-(A.7) from [8] of hydrodynamic values with time-dependent coefficients $\hat{C}_{i}=\hat{C}_{i}(t), i=1, \cdots, 50$. Coefficients $\hat{C}_{i}=\hat{C}_{i}(t)$ are derived from the condition that hydrodynamic values satisfy boundary conditions (2.14)-(2.16)

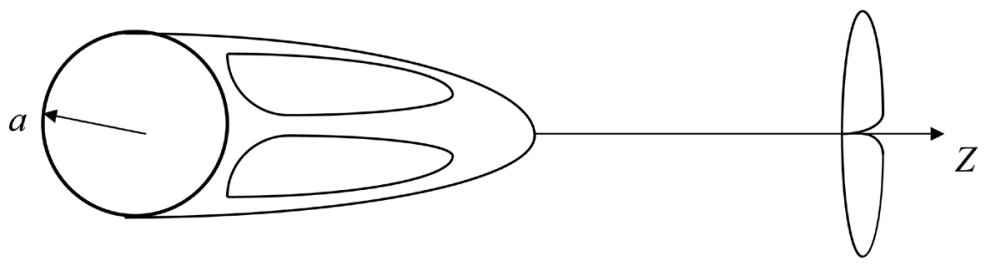

(a)

(b)

Figure 3. (a) Schematic representation of recirculating zone in near wake behind the sphere; (b) Schematic representation of vortex ring in far wake. 
and nonstationary Equations (2.9)-(2.11). Executing the sequence of transformations, which in Section 4 led us to the closed algebraic set of twenty equations, we obtain a closed nonstationary set of twenty equations denoted hereafter by $S 20$. Sixteen algebraic Equations (A.8), (A.9), and (A.13) from [8] supplemented with three algebraic Equation (4.12), remain unchanged when passing from the stationary problem to the nonstationary one. However, Equation (4.11) changes to

$$
\frac{\partial \hat{C}_{5}}{\partial \hat{t}}+\hat{C}_{19}=0
$$

where $t=\left(\operatorname{Re} a / 2 U_{0}\right) \hat{t}$.

Expressions (4.4)-(4.10) with time-dependent coefficients $\hat{C}_{i}=\hat{C}_{i}(t), i=1, \cdots, 20$, define distributions of principle hydrodynamic values. The $S 20$ set is applied to study the time history of coefficients $\hat{C}_{i}(t)=\hat{C}_{i}^{(0)}(t)=\hat{\bar{C}}_{i}^{(0)}+\delta \hat{C}_{i}^{R(0)}(t), i=1, \cdots, 20$. The $\hat{\bar{C}}_{i}^{(0)}, i=1, \cdots, 20$ stationary solution is described in Section 4. Functions $\delta \hat{C}_{i}^{R(0)}(t)$ give deviations from the stationary solution $\hat{\bar{C}}_{i}^{(0)}, i=1, \cdots, 20$.

As revealed by numerical integration of the $S 20$ set, solution $\hat{\bar{C}}_{i}^{(0)}, i=1, \cdots, 20$, remains asymptotically stable up to certain critical $\mathrm{Re}_{0}^{*}$ value, $\operatorname{Re}_{0}^{*}=129.1$. This means that the $\delta \hat{C}_{i}^{R(0)}(t)$ small deviations from the stationary solution $\hat{\bar{C}}_{i}^{(0)}, i=1, \cdots, 20$, are damped at $\operatorname{Re}<\operatorname{Re}^{*}$. The passage of $\operatorname{Re}_{0}^{*}$ is accompanied by the $\hat{\bar{C}}_{i}^{(0)}, \quad i=1, \cdots, 20$, solution stability loss. Starting with $t=0$, the $\delta \hat{C}_{i}^{R(0)}(t), \quad i=1, \cdots, 20$, small axisymmetric deviations begin to increase exponentially at $\operatorname{Re}>\operatorname{Re}_{0}^{*}$. The solid curve in Figure 4 is the time history of $\hat{C}_{20}^{(0)}(t)$ at $\operatorname{Re}=180$. The $\delta \hat{C}_{i}^{R(0)}(t), i=1, \cdots, 20$, deviations grow up to time $t_{*}>0$. At $t=t_{*}$, the $\delta \hat{C}_{i}^{R(0)}(t)$ solution is cut off. Why the solution to the $S 20$ set terminates at $t=t_{*}$ is best explained in terms of trajectories on the phase plane $\hat{C}_{5}^{(0)}, \hat{C}_{19}^{(0)}$ (Figure 6 in [8]). It turned out that at $t>t_{*}$, the $S 20$ set becomes unsuitable for modeling evolution of the physical system.

Time history of the $\hat{C}_{20}^{(0)}(t)$ coefficient at $0<t<t_{*}$ creates time dependence at the distribution of particledensity flux (4.8). The distribution (4.8) corresponds to observed evolution of the periphery of the recirculating zone in the wake behind a sphere [10] [11]. In accordance with experiment, starting with $t=0$, and up to $t=t_{*}$, the periphery of the recirculating zone in the wake behind a sphere moves translationally, receding from the sphere. Starting with $t=t_{*}$ the periphery of the recirculating zone moves back towards the sphere [10] [11].

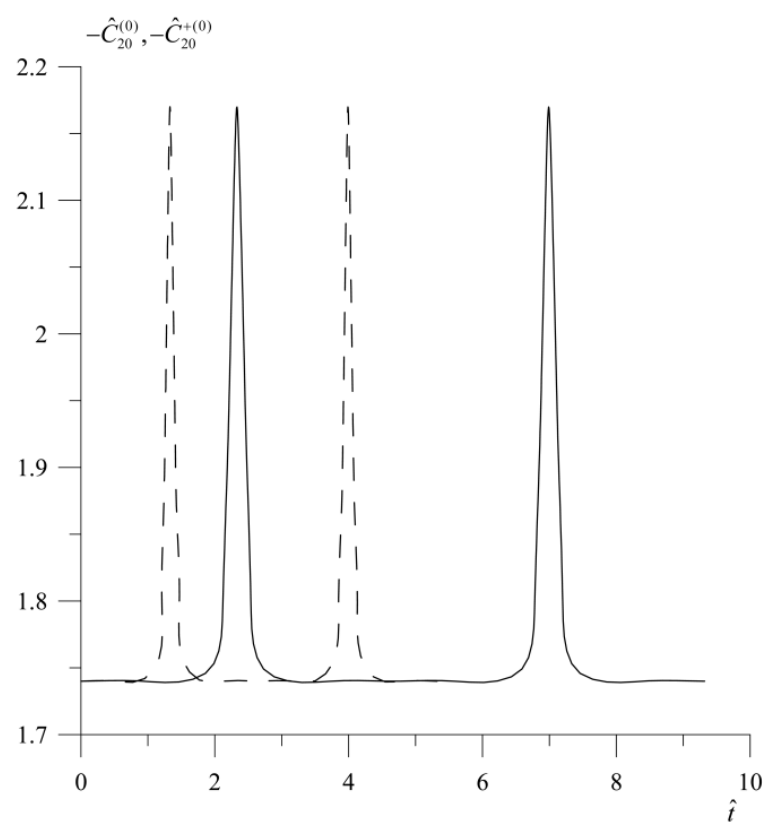

Figure 4. Time evolution of $-\hat{C}_{20}^{(0)} \cdot 10^{3}$ and $-\hat{C}_{20}^{+(0)} \cdot 10^{3}$, Re $=180$. Solid curve corresponds to $\alpha$-ensemble, dashed curve corresponds to $\beta$-ensemble. $\hat{t}_{*}=2.333$ for $\alpha$-ensemble, $\hat{t}_{*}=1.333$ for $\beta$-ensemble. 
Suppose that at time $t=t_{*}$, velocities of all gas particles flowing around the sphere reversed their direction, and the boundary conditions were also reversed. Then the periphery of recirculating zone in the wake behind the sphere starts moving back towards the sphere at $t=t_{*}$. As proven in [6], macroscopic motion of the gas with reversed velocities of its constituent particles is governed by the reverse equations for pair functions. So, observed reverse motion of the periphery of recirculating zone should be described by means of the reverse equations.

However, upon reversing velocities of all gas particles and the boundary conditions, certain details of macroscopic motion of the gas disagree with experiment [10] [11]. For example, upon reversal of gas-particle velocities, the gas involved in vortex motion starts circulating in the opposite direction. However, all gas particles cannot be set in reverse motion at $t=t_{*}$. Nevertheless macroscopic reversal of all gas-particle velocities and boundary conditions is by no means the only way of initiating reverse motion in the wake as a whole. At $t=t_{*}$, reverse motion in the physical system starts spontaneously [10] [11], i.e., without any interference from outside. Insofar as the reverse equations apply to a vortex propagating backward upon reversal of all gas-particle velocities, it is reasonably assumed that the same equations apply to a vortex set in reverse motion in some other way.

Both the direct and the reverse multimoment hydrodynamics equations are specified by Equations (54)-(56) in [5]. However, expressions for reverse nonprinciple hydrodynamic values $p_{i j}^{+v}$ and $q_{i}^{+G v}$ are specified by Equation (2.7), in which each term of the right-hand side has opposite sign [6].

Executing the sequence of transformations, which led us to the closed nonstationary set $S 20$, we obtain a closed nonstationary set of twenty equations for coefficients $\hat{C}_{i}^{+}, i=1, \cdots, 20$, denoted hereafter by $S^{+} 20$. Sixteen algebraic Equations (A.8), (A.9), and (A.13) from [8] supplemented with three algebraic equations (4.12) remain unchanged in going from the direct to the reverse nonstationary problem. However, Equation (5.2) changes to

$$
\frac{\partial \hat{C}_{5}^{+}}{\partial \hat{t}^{+}}-\hat{C}_{19}^{+}=0
$$

It turned out that the $\hat{C}_{i}^{+}\left(t^{+}\right)=\hat{C}_{i}^{+(0)}\left(t^{+}\right), i=1, \cdots, 20$, solution to the $S^{+} 20$ set existed in the neighborhood of the cut-off point. According to [12] [13], the law of large numbers is violated near singular points (bifurcations, regions of the coexistence of several stable solutions, etc.), and large spontaneous fluctuations may appear in the system. In conformity with [12] [13], a large spontaneous fluctuation causes the transfer of the system from the cut-off point to the $\hat{C}_{i}^{+(0)}\left(t^{+}\right), i=1, \cdots, 20$, solution at time $t=t_{*}$. Starting with $t=t_{*}$, axisymmetric deviations $\delta \hat{C}_{i}^{+R(0)}\left(t^{+}\right), i=1, \cdots, 20$, begin to decay exponentially, $\hat{C}_{i}^{+(0)}\left(t^{+}\right)=\hat{\bar{C}}_{i}^{(0)}+\delta \hat{C}_{i}^{+R(0)}\left(t^{+}\right)$. At time $t=2 t_{*}$, the $\hat{C}_{i}^{+(0)}\left(t^{+}\right)$solution reaches the neighborhood of the $\bar{C}_{i}^{(0)}, i=1, \cdots, 20$, stationary solution. At time $t=2 t_{*}$ further evolution of the $\hat{C}_{i}^{+(0)}\left(t^{+}\right), i=1, \cdots, 20$, solution finishes. The $\hat{C}_{i}^{(0)}(t), i=1, \cdots, 20$, solution exists at $0 \leq t \leq t_{*}$, the $\hat{C}_{i}^{+(0)}\left(t^{+}\right), i=1, \cdots, 20$, solution exists at $t_{*} \leq t \leq 2 t_{*}, t^{+}=-t+2 t_{*}$. At any time instant $0 \leq t_{0} \leq t_{*}$ :

$$
\left.\hat{C}_{i}^{+(0)}\left(t^{+}\right)\right|_{t^{+}=t_{0}}=\left.\hat{C}_{i}^{(0)}(t)\right|_{t=t_{0}}
$$

A large spontaneous fluctuation causes the transfer of the system from the $\hat{C}_{i}^{+(0)}\left(t^{+}\right), i=1, \cdots, 20$, solution to the $\hat{\bar{C}}_{i}^{(0)}, i=1, \cdots, 20$, solution at time $t=2 t_{*}$. The $\hat{\bar{C}}_{i}^{(0)}, i=1, \cdots, 20$, solution is unstable. It follows that small axisymmetric deviations $\delta \hat{C}_{i}^{+R(0)}\left(t^{+}\right), i=1, \cdots, 20$, begin to grow starting with time $t=2 t_{*}$. This process is repeated periodically. So, we obtained the solution $\hat{C}_{i}^{(0)}(t), \hat{C}_{i}^{+(0)}\left(t^{+}\right), i=1, \cdots, 20$, referred hereinafter as $\mathrm{Sol}_{0}$.

Numerical integration of the $S 20, S^{+} 20$ set draws the time history of coefficients $\hat{C}_{i}^{(0)}(t), \hat{C}_{i}^{+(0)}\left(t^{+}\right)$. The time history of $\hat{C}_{20}^{(0)}(t)$ and $\hat{C}_{20}^{+(0)}\left(t^{+}\right)$shown in Figure 4 by a solid line corresponds to initial deviation $\delta \hat{C}_{19}^{R(0)}(t=0)=10^{-9}$. Deviations $\delta \hat{C}_{i}^{(0) R}(t=0) \quad i=1, \cdots, 18,20$ are dependent quantities and can be calculated from the $S 20$ set, in which equation (5.2) is substituted by equation $\delta \hat{C}_{19}^{(0) R}(t=0)=10^{-9}$. Calculation gives $\delta \hat{C}_{i}^{R(0)}(t=0) \sim 10^{-9}-10^{-10}, i=1, \cdots, 20$. The equations of the $S 20, S^{+} 20$ set were integrated with the accuracy $\Delta \varepsilon$ of the order of $\delta \hat{C}_{19}^{R(0)}(t=0)$. Accuracy $\Delta \varepsilon \sim 10^{-9}$ is a measure of fluctuations of hydrodynamic values at thermodynamic equilibrium. The time history of the coefficients $\delta \hat{C}_{20}^{R(0)}(t)$ and $\delta \hat{C}_{20}^{+R(0)}\left(t^{+}\right)$from Figure 4 corresponds to axisymmetric pulsations of the periphery of recirculating zone in the wake behind a 
sphere. The half-period of pulsations $t_{*}$ was estimated at approximately one minute at $a \sim 1 \mathrm{sm}$ and $U_{0} \sim 10 \mathrm{sm} / \mathrm{s}$.

The dashed curve in Figure 4 is the $\delta \hat{C}_{20}^{R(0)}(t), \delta \hat{C}_{20}^{+R(0)}\left(t^{+}\right)$dependence calculated at $\delta \hat{C}_{19}^{R(0)}(t=0)=10^{-6}$ with accuracy $\Delta \varepsilon \sim 10^{-9}$. Thus, the $S 20, S^{+} 20$ nonlinear set "draws apart” initially close trajectories. This sensitivity to initial conditions was called the Loretz "butterfly effect” [14].

The multimoment hydrodynamics equations [5], as well as the classic hydrodynamic equations, govern space and time evolution of the whole ensemble of systems (Gibbs ensemble) rather than of some individual system. All the microscopic parameters of each individual system are compatible with the initial macroscopic parameters which are present not in the form of particular values but in the form of intervals allowing for their possible fluctuations [15]. Thus, each statistical coefficient, $\hat{C}_{i}^{(0)}(t), i=1, \cdots, 20$, is a linear combination of a great many dynamic coefficients $\hat{C}_{i, j}^{D(0)}(t)$, denote their number by $K$ which can be infinitely large

$$
\hat{C}_{i}^{(0)}(t)=\frac{1}{K} \sum_{j=1}^{K} \hat{C}_{i, j}^{D(0)}(t) \quad K \gg 1
$$

Dynamic coefficients $\hat{C}_{i, j}^{D(0)}(t)$ are calculated within the classic mechanics. Fluctuation $\delta \hat{C}_{i}^{(0)}(t)$ at any time is defined as a difference between the dynamic and statistical coefficients,

$\delta \hat{C}_{i}^{(0)}(t)=\delta \hat{C}_{i, j}^{D(0)}(t)=\hat{C}_{i, j}^{D(0)}(t)-\hat{C}_{i}^{(0)}(t)$, or which is the same, as a difference between two arbitrary dynamic coefficients, $\delta \hat{C}_{i}^{(0)}(t)=\hat{C}_{i, k}^{D(0)}(t)-\hat{C}_{i, l}^{D(0)}(t)$.

Consider the range of the system parameters $\left(\operatorname{Re}<\mathrm{Re}_{0}^{*}\right)$ within which the equations for statistical coefficients have stable solution $\hat{\bar{C}}_{i}^{(0)}, i=1, \cdots, 20$. Within this range, the overwhelming majority of dynamic coefficients $\hat{C}_{i, j}^{D(0)}(t)$ passing in the immediate vicinity of $\hat{\bar{C}}_{i}^{(0)}$ at $t=0 \quad\left(\delta \hat{C}_{i}^{(0)}(t=0)=\hat{C}_{i, j}^{D(0)}(t=0)-\hat{\bar{C}}_{i}^{(0)} \ll 1\right)$ will remain in the vicinity of $\hat{\bar{C}}_{i}^{(0)}$ indefinitely long $\left(\delta \hat{C}_{i}^{(0)}(t) \simeq \delta \hat{C}_{i}^{(0)}(t=0)\right)$. Statistical solution $\hat{\bar{C}}_{i}^{(0)}$, $i=1, \cdots, 20$, describes most of the dynamic trajectories $\hat{C}_{i, j}^{D(0)}(t), j=1, \cdots, K$, with the accuracy $\delta \hat{C}_{i}^{(0)}(t=0) \ll 1$.

The situation in the unstable range $\left(R e>R_{0}^{*}\right)$ is radically different. Consider two ensembles of systems. The first $\alpha$-ensemble at $t=0$ incorporates systems with coefficients $\hat{C}_{i, j}^{D(\alpha)}(t=0), j=1, \cdots, K_{\alpha}, K_{\alpha} \gg 1$ within small vicinity $\delta \hat{C}_{i}^{(\alpha)}(t=0) \ll 1$ near $\alpha$-solution $\hat{\bar{C}}_{i}^{(\alpha)}(t=0), i=1, \cdots, 20$, here, $\hat{\bar{C}}_{19}^{(\alpha)}(t=0)=10^{-9}$. The second $\beta$-ensemble at $t=0$ incorporates systems with coefficients $\hat{C}_{i, j}^{D(\beta)}(t=0), j=1, \cdots, K_{\beta}, K_{\beta} \gg 1$ within small vicinity $\delta \hat{C}_{i}^{(\beta)}(t=0) \ll 1$ near $\beta$-solution $\hat{\bar{C}}_{i}^{(\beta)}(t=0), i=1, \cdots, 20$, here, $\hat{\bar{C}}_{19}^{(\beta)}(t=0)=10^{-6}$. Let $\left.\left.\hat{C}_{i}^{(\alpha)}(t)\right|_{t=0} \simeq \hat{C}_{i}^{(\beta)}(t)\right|_{t=0}, i=1, \cdots, 20$, at time $t=0$. Time history of the coefficients $\hat{C}_{20}^{(\alpha)}(t)$ and $\hat{C}_{20}^{(\beta)}(t)$ is drawn in Figure 4 (solid and dashed curves). Initially close trajectories diverge. It immediately follows that each dynamic $j$-coefficient, $\hat{C}_{i, j}^{D(0)}(t)$, strictly speaking, behaves in its own way. There is no unique $\hat{C}_{i}^{(0)}(t)$, $i=1, \cdots, 20$, which describes any dynamic coefficient from the set $\hat{C}_{i, j}^{D(0)}(t), j=1, \cdots, K, K \gg 1$, for the whole ensemble with the accuracy $\delta C_{i}^{(0)}(t=0) \ll 1$. The Gibbs ensemble disintegrates.

Disintegration of the Gibbs ensemble in the unstable region suggests the follows. The multimoment hydrodynamics Equations (2.9)-(2.11) governing the Gibbs ensemble as a whole are invalid in the region where solutions to these equations become unstable. The $p_{i j}^{v}$ and $q_{i}^{G v}$ dissipation moments (2.7) are also true for the ensemble as a whole. Deviations $\delta \hat{C}_{i}^{R(0)}(t), i=1, \cdots, 20$, are nothing but fluctuations of stationary coefficients $\hat{\bar{C}}_{i}^{(0)}$, $i=1, \cdots, 20$.

Fluctuation $\delta \hat{C}_{i}^{R(0)}(t)$ of some coefficient $\hat{\bar{C}}_{i}^{(0)}$, namely, $\hat{\bar{C}}_{19}^{(0)}$, is preset at $t=0$, for example, $\delta \hat{C}_{19}^{R(0)}(t=0)=b_{19}$. Fluctuations $\delta \hat{C}_{i}^{R(0)}(t=0), i=1, \cdots, 18,20$, of other coefficients are calculated from the $S 20$ set, in which Equation (5.2) is substituted by equation $\delta \hat{C}_{19}^{R(0)}(t=0)=b_{19}$. Time evolution of the so formulated fluctuation is approximated by solution of the $S 20, S^{+} 20$ set. Fluctuation of any hydrodynamic value at any instant $t$ and any point $\mathbf{x}$ is calculated from Equations (4.4)-(4.10). The fluctuations are interre- 
lated in time and space. In [8], these interrelated fluctuations have been termed regular. However, there is a factor always present in real physical systems. It is the spontaneous chaotic fluctuation [16]. Spontaneous fluctuations are random independent events. Thus, fluctuation $\delta \hat{C}_{i}^{(0)}(t)$ of the $\hat{\bar{C}}_{i}^{(0)}$ statistical coefficient is

$$
\delta \hat{C}_{i}^{(0)}(t)=\delta \hat{C}_{i}^{(0) R}(t)+\delta \hat{C}_{i}^{(0) S}(t)
$$

After the attainment of the first critical value $\operatorname{Re}_{0}^{*}$, the $\mathrm{Sol}_{0}$ solution to the multimoment hydrodynamics equations loses its stability. The conservation laws (2.9)-(2.11) governing the Gibbs ensemble as a whole become invalid. Regular fluctuations alone cannot provide for fulfillment of Equations (2.9)-(2.11). Strictly speaking, to solve the unstable problem accurately, one needs to switch from the statistical to the dynamic level of description and apply the equations of classical mechanics modeling the dynamics of each individual gas particle. However, numerical integration of the classical mechanics equations for a tremendous number of particles (which is sometimes infinite) is an extremely arduous problem. This line of attack seems ill advised.

In [17], when modeling an individual system, each hydrodynamic value in the equations of conservation was supplemented with its spontaneous fluctuation component. As was done in [17], let us attract spontaneous fluctuations. With spontaneous fluctuations taken into account, equations of conservation (2.9)-(2.11) are satisfied in the case of both direct and reverse multimoment hydrodynamics equations. To satisfy the Equations (2.9)-(2.11), the contribution of the time derivative of regular fluctuations $\delta \hat{C}_{i}^{R(0)}(t)$ must be counterbalanced by the contribution of time and space derivatives of spontaneous fluctuations $\delta \hat{C}_{i}^{S(0)}(t)$. Namely

$$
\frac{\partial \delta \hat{C}_{i}^{R(0)}(t)}{\partial \hat{t}}+\frac{\partial \delta \hat{C}_{i}^{S(0)}(t)}{\partial \hat{t}}+f_{i}=0 \quad i=1,2,3,4,6,7,14,19,20
$$

The $f_{i}$ functions incorporate space derivatives of spontaneous fluctuations. In the case of reverse equations of conservation, besides of Equation (5.7), the change in third equation of set (4.12) (namely, the replacement of $\hat{C}_{20}$ by $-\hat{C}_{20}$ ) must be counterbalanced by the contribution of spontaneous fluctuations.

Let us now transform Equation (5.7) to the dimensional form and assess the order of spontaneous fluctuations varying on time and space scales $\tilde{\tau}$ and $\tilde{l}$. Possible large-scale spontaneous fluctuations with $\tilde{\tau} \sim \operatorname{Re} a / U_{0}$ and $\tilde{l} \sim a$ are of the order of large-scale regular fluctuations. Realistic small scale spontaneous fluctuations with $\tilde{\tau} \ll \operatorname{Re} a / U_{0}$ and $\tilde{l} \ll a$ contribute very little, if at all, to the distributions of hydrodynamic values. However, their time and space gradients are of the basic order of magnitude.

\section{Interpretation of System Stability Loss in Terms of Pair Entropy}

Let us mentally circumscribe a sphere of radius $A>a$ around the sphere of radius $a$. Let us term the gas confined between the surfaces of the coaxial spheres the physical system or simply the system. Let us now turn the $A$ radius of the larger sphere to infinity. In pursuance of the $A \gg a$ condition, the number of particles clinging to the surface of the sphere of radius $A$ at any instant is negligibly small compared to the total number of particles in the system. The $A \gg a$ condition makes it possible to form quasi-isolated system. The processes occurring in a quasi-isolated system are studied without regard for interaction of this system with the ambient medium [16]. According to [16], let us neglect fluctuations of hydrodynamic values characterizing the system as a whole, which arise due to permeability of the external sphere of radius $A$. In pursuance of the $A \gg a$, these fluctuations cannot alter the general physical pattern of the processes.

When deriving equations of entropy conservation (A.6), we reasoned from the concept of a Gibbs ensemble of systems. When modeling an individual system, each hydrodynamic value in the equations of conservation should be supplemented with its fluctuation component [17]. Let us correct Equation (A.9) for the $\delta S_{p}^{(0)}(t, \mathbf{x})$ fluctuation of the $\bar{S}_{p}^{(0)}(t, \mathbf{x})$ pair entropy, $S_{p}^{(0)}(t, \mathbf{x})=\bar{S}_{p}^{(0)}(t, \mathbf{x})+\delta S_{p}^{(0)}(t, \mathbf{x})$. Here, superscript (0) corresponds to the 0 -solution to the multimoment hydrodynamics equations. Integrating the resulting equations with respect to $\mathbf{x}$ over the volume of the system $V$ yields

$$
\frac{\partial S_{p}^{(0)}(t)}{\partial t}+\Delta_{E X} S_{p}^{(0)}(t)=\Delta_{I N} S_{p}^{(0)}(t) \quad S_{p}^{(0)}(t)=\bar{S}_{p}^{(0)}(t)+\delta S_{p}^{(0)}(t)
$$

In accordance with (6.1), evolution of the $S_{p}^{(0)}(t)$ pair entropy is defined by two factors, by the $\Delta_{I N} S_{p}^{(0)}(t)$ entropy production in the system and the $\Delta_{E X} S_{p}^{(0)}(t)$ entropy outflow through the surface confining the system.

Balance Equation (6.1) is valid for $\bar{S}_{p}^{(0)}(t)$ and $\delta S_{p}^{(0)}(t)$ individually. The $\bar{S}_{p}^{(0)}(t)$ function characterizes 
the ensemble of systems as a whole. The second term of the left-hand side of Equation (6.1) is integrated over surface confining volume $V$ (over the surface of the spheres of radii $A$ and $a$ ). Note that the physical meaning of the Boltzmann entropy was established without resorting to the concept of a Gibbs ensemble [18]. Inequalities (A.3) were also derived without invoking this concept. Thus, inequalities (A.3) are also valid for $S_{p}(t), S_{p p}(t)$, and $S_{1}(t)$, and entropies $S_{p}(t), S_{p p}(t)$, and $S_{1}(t)$ also convey the meaning of volume occupied by the system in the $\Gamma$-space.

In terms of Equation (5.6), the $\delta S_{p}^{(0)}(t, \mathbf{x})$ fluctuation can be written as

$$
\delta S_{p}^{(0)}(t)=\delta S_{p}^{R(0)}(t)+\delta S_{p}^{S(0)}(t)
$$

Here, superscripts " $R$ " and " $S$ " mark the contribution of regular and spontaneous fluctuations to the entropy.

Explicit analytical expressions for space distributions of principle hydrodynamic values (4.4)-(4.10) contain dimensionless parameters $\left(\mathrm{Ma}^{2}\right.$ and $\left.\mathrm{Re}\right)$. The $\mathrm{Re}$-dependence is contained within the $\hat{C}_{i}=\hat{C}_{i}(\mathrm{Re})$,

$i=1, \cdots, 20$, coefficients. In terms of Equations (4.4-4.10), the components of Equation (6.1) can be written in the form of an infinite series in $\mathrm{Ma}^{2}$

$$
\begin{gathered}
S_{p}^{(0)}(t)=\sum_{k=0}^{\infty} S_{p}^{(0, k)}(t)=S_{0} \sum_{k=0}^{\infty}\left(\mathrm{Ma}^{2}\right)^{k} \hat{S}_{p}^{(0, k)}(t) \\
\Delta_{I N(E X)} S_{p}^{(0)}(t)=\sum_{k=0}^{\infty} \Delta_{I N(E X)} S_{p}^{(0, k)}(t)=\frac{S_{0}}{\tau_{0} \operatorname{Re}^{2}} \sum_{k=0}^{\infty}\left(\mathrm{Ma}^{2}\right)^{k+1} \Delta_{I N(E X)} \hat{S}_{p}^{(0, k)}(t)
\end{gathered}
$$

Here, $S_{0}=k n_{0} v_{0}, v_{0}=(4 / 3) \pi a^{3}, \tau_{0}=2 \eta_{0} / n_{0} k T_{0}$. Substituting distributions of hydrodynamic values (3.6), (3.7), (4.4)-(4.10) into (A.7), (A.10) and integrating the resulting expressions with respect to $\mathbf{x}$ over the volume $V$ yield

$$
\begin{aligned}
& S_{p}^{(0,0)}=-\frac{k N_{0}}{2}\left\{\ln \left[\frac{n_{0}}{2}\left(\frac{m}{2 \pi k T_{0}}\right)^{3}\right]-3\right\} \\
& S_{p}^{(0,1)}=-\frac{k\left(N-N_{0}\right)}{2}\left\{\ln \left[\frac{n_{0}}{2}\left(\frac{m}{2 \pi k T_{0}}\right)^{3}\right]+1\right\}+\frac{E-E_{0}}{T_{0}} \\
& \bar{S}_{p}^{(0,2)}+\delta S_{p}^{R(0,2)}(t)=k n_{0} v_{0} \operatorname{Ma}^{4}\left(\hat{\bar{S}}_{p}^{(0,2)}+\delta \hat{S}_{p}^{R(0,2)}(t)\right) \\
& \hat{\bar{S}}_{p}^{(0,2)}+\delta \hat{S}_{p}^{R(0,2)}(t)=-\left\{(4131 / 640) \hat{C}_{1}^{2}-(1 / 5) \hat{C}_{1} \hat{C}_{2}-2 \hat{C}_{1} \hat{C}_{3}-(39 / 10) \hat{C}_{1} \hat{C}_{5}-11 \hat{C}_{1} \hat{C}_{6}+(3 / 4) \hat{C}_{1} \hat{C}_{8}-(123 / 16)\right. \\
& \times \hat{C}_{1} \hat{C}_{18}-(871 / 160) \hat{C}_{1} \hat{C}_{19}+(72136 / 17325) \hat{C}_{1}\left(\operatorname{Re} \hat{C}_{20}\right)^{2}-90 \hat{C}_{1} \hat{C}_{20}-(7097 / 640) \hat{C}_{1}+(4 / 5) \hat{C}_{2}^{2}+(6 / 5) \hat{C}_{2} \hat{C}_{5} \\
& +(2 / 5) \hat{C}_{2} \hat{C}_{19}-(2144 / 17325) \hat{C}_{2}\left(\operatorname{Re} \hat{C}_{20}\right)^{2}+(19 / 80) \hat{C}_{2}+12 \hat{C}_{3}^{2}+12 \hat{C}_{3} \hat{C}_{6}+4 \hat{C}_{3} \hat{C}_{19}-(544 / 225) \hat{C}_{3}\left(\operatorname{Re} \hat{C}_{20}\right)^{2} \\
& +(201 / 32) \hat{C}_{3}+\hat{C}_{4}^{2}+\hat{C}_{4} \hat{C}_{7}-(3 / 2)\left(\hat{C}_{4} / \operatorname{Re}\right)+(24 / 5) \hat{C}_{5}^{2}-(24 / 5) \hat{C}_{5} \hat{C}_{8}+(18 / 5) \hat{C}_{5} \hat{C}_{18}+(9 / 5) \hat{C}_{5} \hat{C}_{19} \\
& -(192 / 385) \hat{C}_{5}\left(\operatorname{Re} \hat{C}_{20}\right)^{2}+192 \hat{C}_{5} \hat{C}_{20}+(3 / 5) \hat{C}_{5}+9 \hat{C}_{6}^{2}+6 \hat{C}_{6} \hat{C}_{19}-(1024 / 225) \hat{C}_{6}\left(\operatorname{Re} \hat{C}_{20}\right)^{2}+(287 / 32) \hat{C}_{6} \\
& +2 \hat{C}_{7}^{2}-(2 / 3) \hat{C}_{7} \hat{C}_{9}-4\left(\hat{C}_{7} / \operatorname{Re}\right)+2 \hat{C}_{8}^{2}-(3 / 5) \hat{C}_{8} \hat{C}_{18}-(3 / 10) \hat{C}_{8} \hat{C}_{19}+(1024 / 17325) \hat{C}_{8}\left(\operatorname{Re} \hat{C}_{20}\right)^{2}-96 \hat{C}_{8} \hat{C}_{20} \\
& +(3 / 80) \hat{C}_{8}+(1 / 4) \hat{C}_{9}^{2}+(63 / 8) \hat{C}_{18}^{2}+(123 / 40) \hat{C}_{18} \hat{C}_{19}-(10336 / 5775) \hat{C}_{18}\left(\operatorname{Re} \hat{C}_{20}\right)^{2}+72 \hat{C}_{18} \hat{C}_{20}+(1641 / 160) \hat{C}_{18} \\
& +(219 / 160) \hat{C}_{19}^{2}-(35152 / 17325) \hat{C}_{19}\left(\operatorname{Re} \hat{C}_{20}\right)^{2}+36 \hat{C}_{19} \hat{C}_{20}+(339 / 64) \hat{C}_{19}+(24293408768 / 49517887419) \\
& \times\left(\operatorname{Re} \hat{C}_{20}\right)^{4}-(768 / 77) \operatorname{Re} \hat{C}_{20}^{3}+2400 \hat{C}_{20}^{2}+12 \hat{C}_{20}+(240968512 / 52026975)\left(\operatorname{Re} \hat{C}_{20}\right)^{2}+(27 / 4)\left(1 / \operatorname{Re}{ }^{2}\right) \\
& \left.+\ln 2\left[(1107 / 160) \hat{C}_{1}-(27 / 8) \hat{C}_{3}-(45 / 8) \hat{C}_{6}-(243 / 40) \hat{C}_{18}-(261 / 80) \hat{C}_{19}\right]\right\}
\end{aligned}
$$




$$
\begin{aligned}
& \Delta_{I N} \bar{S}_{p}^{(0,1)}+\Delta_{I N} \delta S_{p}^{R(0,1)}(t)=\frac{k n_{0} v_{0} \operatorname{Ma}^{4}}{\tau_{0} \operatorname{Re}^{2}}\left(\Delta_{I N} \hat{\bar{S}}_{p}^{(0,1)}+\Delta_{I N} \delta \hat{S}_{p}^{R(0,1)}(t)\right) \\
& \Delta_{I N} \hat{\bar{S}}_{p}^{(0,1)}+\Delta_{I N} \delta \hat{S}_{p}^{R(0,1)}(t)=36+(8192 / 45)\left(\operatorname{Re} \hat{C}_{20}\right)^{2} \\
& \Delta_{I N} \bar{S}_{p}^{(0,2)}+\Delta_{I N} \delta S_{p}^{R(0,2)}(t)=\frac{k n_{0} v_{0} \mathrm{Ma}^{6}}{\tau_{0} \operatorname{Re}^{2}}\left(\Delta_{I N} \hat{\bar{S}}_{p}^{(0,2)}+\Delta_{I N} \delta \hat{S}_{p}^{R(0,2)}(t)\right) \\
& \Delta_{I N} \hat{\bar{S}}_{p}^{(0,2)}+\Delta_{I N} \delta \hat{S}_{p}^{R(0,2)}(t) \\
& +-\left[-(28144 / 5775) \hat{C}_{1}\left(\operatorname{Re} \hat{C}_{20}\right)^{2}-(27 / 40) \hat{C}_{1}+216 \hat{C}_{2}^{2}+(1296 / 5) \hat{C}_{2} \hat{C}_{5}+(144 / 5) \hat{C}_{2} \hat{C}_{19}\right. \\
& +(24128 / 3465) \hat{C}_{2}\left(\operatorname{Re} \hat{C}_{20}\right)^{2}-3456 \hat{C}_{2} \hat{C}_{20}+(27 / 5) \hat{C}_{2}+360 \hat{C}_{3}^{2}+288 \hat{C}_{3} \hat{C}_{6}+96 \hat{C}_{3} \hat{C}_{19} \\
& +(352 / 25) \hat{C}_{3}\left(\operatorname{Re} \hat{C}_{20}\right)^{2}+(489 / 8) \hat{C}_{3}+60 \hat{C}_{4}^{2}+48 \hat{C}_{4} \hat{C}_{7}+(1944 / 25) \hat{C}_{5}^{2}+(432 / 25) \hat{C}_{5} \hat{C}_{19} \\
& +(49152 / 9625) \hat{C}_{5}\left(\operatorname{Re} \hat{C}_{20}\right)^{2}-(10368 / 5) \hat{C}_{5} \hat{C}_{20}+(81 / 25) \hat{C}_{5}+(288 / 5) \hat{C}_{6}^{2}+(192 / 5) \hat{C}_{6} \hat{C}_{19} \\
& +(4096 / 375) \hat{C}_{6}\left(\operatorname{Re} \hat{C}_{20}\right)^{2}+(126 / 5) \hat{C}_{6}+(48 / 5) \hat{C}_{7}^{2}+(64 / 5) \hat{C}_{11}-64 \hat{C}_{12} \\
& +(216 / 25) \hat{C}_{19}^{2}+(76288 / 28875) \hat{C}_{19}\left(\operatorname{Re} \hat{C}_{20}\right)^{2}-(1152 / 5) \hat{C}_{19} \hat{C}_{20}+(297 / 25) \hat{C}_{19} \\
& +(37148916973568 / 3163642807325)\left(\operatorname{Re} \hat{C}_{20}\right)^{4}+(29212672 / 17325) \hat{C}_{20}\left(\operatorname{Re} \hat{C}_{20}\right)^{2} \\
& \left.+(1262774528 / 58963905)\left(\operatorname{Re} \hat{C}_{20}\right)^{2}+13824 \hat{C}_{20}^{2}-(936 / 5) \hat{C}_{20}+69948 / 9625\right]
\end{aligned}
$$

Here, $N_{0}=n_{0} V, \quad E_{0}=(3 / 2) n_{0} k T_{0} V, \quad N=\int n \mathrm{~d} \mathbf{x}, \quad E=(3 / 2) \int n k T \mathrm{~d} \mathbf{x}$. Coefficients $\hat{C}_{i}=\hat{C}_{i}^{(0)}=\bar{C}_{i}^{(0)}(\mathrm{Re})+\delta C_{i}^{R(0)}(t, \mathrm{Re}), i=1, \cdots, 20$, are given in Section 4. The terms of distributions (4.4)-(4.10) proportional to $(a / r)$ give rise to terms proportional to $(A-a)$ and $\ln (A / a) \quad(A \gg a)$ in Equation (6.6) upon integration with respect to $\mathbf{x}$ over volume $V$. That is why, in deriving (6.6), the integration limit for indefinitely increasing terms is changed by putting $1 \leq r \leq 2, \pi \leq \theta \leq 0,2 \pi \leq \varphi \leq 0$.

The $S_{p}^{(0,1)}$ function (6.5) incorporates the total number of particles in the system $N$ and the total thermal energy $E$. Owing to aforesaid condition $(A \gg a)$ and the boundary conditions (2.16) adapted to an individual system, fluctuations of these quantities ( $N$ and $E$ ) can be excluded from consideration. Thus, $\partial S_{p}^{(0,1)} / \partial t=0$. So, pair entropy $S_{p}^{(0,1)}$ accurate to the order of $k n_{0} v_{0} \mathrm{Ma}^{2}$ does not change at time. Hence, the system stability is independent of terms of this order. The study of Equation (6.1) undertaken in [19] has revealed that entropy production in the system $\Delta_{I N} \bar{S}_{p}^{(0,1)}+\Delta_{I N} \delta S_{p}^{R(0,1)}(t)$ (6.7) compensates the entropy outflow through the surface confining the system $\Delta_{E X} \bar{S}_{p}^{(0,1)}+\Delta_{E X} \delta S_{p}^{R(0,1)}(t)$, then, $\Delta_{E X} \delta S_{p}^{S(0,1)}(t)=0$ because the $\Delta_{I N} \delta S_{p}^{S(0,1)}(t)$ term is omitted for the reason to be explained later in this section.

In problem with time independent boundary conditions, entropy balance Equation (6.1) accurate to the order of $k n_{0} v_{0} \mathrm{Ma}^{6} / \tau_{0} \mathrm{Re}^{2}$ assume the form

$$
\frac{\partial\left(\delta S_{p}^{R(0,2)}(t)+\delta S_{p}^{S(0,2)}(t)\right)}{\partial t}+\Delta_{E X}\left(\bar{S}_{p}^{(0,2)}+\delta S_{p}^{R(0,2)}(t)+\delta S_{p}^{S(0,2)}(t)\right)=\Delta_{I N}\left(\bar{S}_{p}^{(0,2)}+\delta S_{p}^{R(0,2)}(t)\right)
$$

As is noted in Section 5, spontaneous fluctuations in large systems tend to be as small as possible. Further, the nearest vicinities of the point at which the solution breaks will be omitted from consideration. In this case, in accordance with the law of large numbers [12] [13], large spontaneous fluctuations can be disregarded. The contempt for large spontaneous fluctuations makes it possible to omit the $\Delta_{I N} \delta S_{p}^{S(0, i)}(t), i=1,2$, terms allowing for small spontaneous fluctuations. However, varying with space and time on the scales $\tilde{l} \ll a$ and $\tilde{\tau} \ll \operatorname{Re} a / U_{0}$, small spontaneous fluctuations keep equations of conservation (6.9) valid. Thus, space and time 
gradients of small spontaneous fluctuations must be retained on the left hand side of Equation (6.9).

Derivative $\partial \delta S_{p}^{R(0,2)}(t) / \partial t$ is calculated from Equation (6.6). One of ways of allowing for the contribution of spontaneous fluctuations $\partial \delta S_{p}^{S(0,2)}(t) / \partial t$ is to introduce a random source [17]. However, numerical integration of the $S 20, S^{+} 20$ set is as much in error as the random source method. Thus, the contribution of spontaneous fluctuations is taken into account involuntary, owing to the error in numerical calculation $\partial \delta S_{p}^{R(0,2)}(t) / \partial t$.

The study of the $\partial \mathbf{S}_{p}^{(0,2)}(t, \mathbf{x}) / \partial \mathbf{x}$ spatial variation of the pair entropy [19] has revealed that the $\Delta_{E X}\left(\bar{S}_{p}^{(0,2)}+\delta S_{p}^{R(0,2)}(t)\right)$ term models entropy removal from the system exclusively through the surface of the solid sphere of radius $a$. By virtue of the $A \gg a$ condition, the $\Delta_{E X} \delta S_{p}^{S(0,2)}(t)$ term models entropy removal by spontaneous fluctuations also through the surface of the sphere. The entropy balance in the solid sphere is maintained by thermal radiation.

Suppose that at time $t=0$, the system produces an entropy fluctuation $\left.\delta S_{p}^{(0,2)}(t)\right|_{t=0}<0$. The $\partial \delta S_{p}^{(0,2)}(t) /\left.\partial t\right|_{t=0}$ values at $\operatorname{Re}$ close to $\operatorname{Re}_{0}^{*}$ are plotted at Figure 5. As revealed by calculations, $\partial \delta S_{p}^{(0,2)}(t) /\left.\partial t\right|_{t=0}>0$ at $\operatorname{Re}<\operatorname{Re}_{0}^{*}$ and $\partial \delta S_{p}^{(0,2)}(t) /\left.\partial t\right|_{t=0}<0$ at $\operatorname{Re}>\operatorname{Re}_{0}^{*}$. Thus, at $\operatorname{Re}<\operatorname{Re}_{0}^{*}$, any fluctuation with $\left.\delta S_{p}^{(0,2)}(t)\right|_{t=0}<0$ generated by the system tends to fade out, whereas at $\operatorname{Re}>\operatorname{Re}_{0}^{*}$, any fluctuation with $\left.\delta S_{p}^{(0,2)}(t)\right|_{t=0}<0$ tends to grow. Time history of $\partial \delta S_{p}^{(0,2)}(t) / \partial t$ is illustrated in Figure 6. From Figure 6 follows that $\partial \delta S_{p}^{(0,2)}(t) / \partial t>0$ at $\operatorname{Re}=129<\operatorname{Re}_{0}^{*}$ and $t \geq 0$. Hence, entropy fluctuations $\left.\delta S_{p}^{(0,2)}(t)\right|_{t=0}<0$ generated by the system die down monotonically, and the entropy of the system $S_{p}^{(0)}(t)$ tends to its stationary value $\bar{S}_{p}^{(0)}$. At $\operatorname{Re}=130>\operatorname{Re}_{0}^{*}$ and $t \geq 0, \quad \partial \delta S_{p}^{(0,2)}(t) / \partial t<0$. Thus, entropy fluctuations $\left.\delta S_{p}^{(0,2)}(t)\right|_{t=0}<0$ produced by the system build up

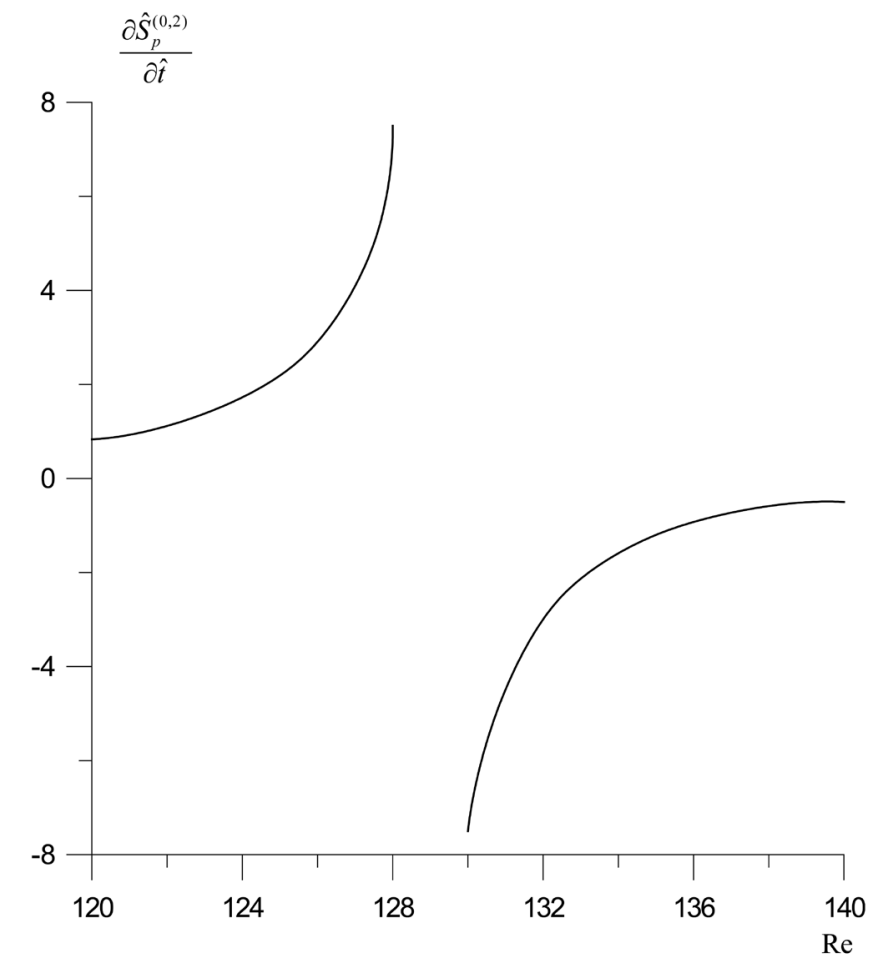

Figure 5. Pair entropy derivative $\partial \hat{S}_{p}^{(0,2)} / \partial \hat{t}=\partial \delta \hat{S}_{p}^{R(0,2)} / \partial \hat{t}$ as a function of $\mathrm{Re}, \operatorname{Re}_{0}^{*}=129.1$. The curves correspond to $\left(\partial \delta \hat{S}_{p}^{R(0,2)}(t) / \partial \hat{t}\right) \cdot 10^{4}$ at time $t=0$. Pair entropy deviation at $t=0$ is taken to be independent of Re: $\left.\delta \hat{S}_{p}^{R(0,2)}(t)\right|_{t=0}=-1.5 \times 10^{-6}$. 


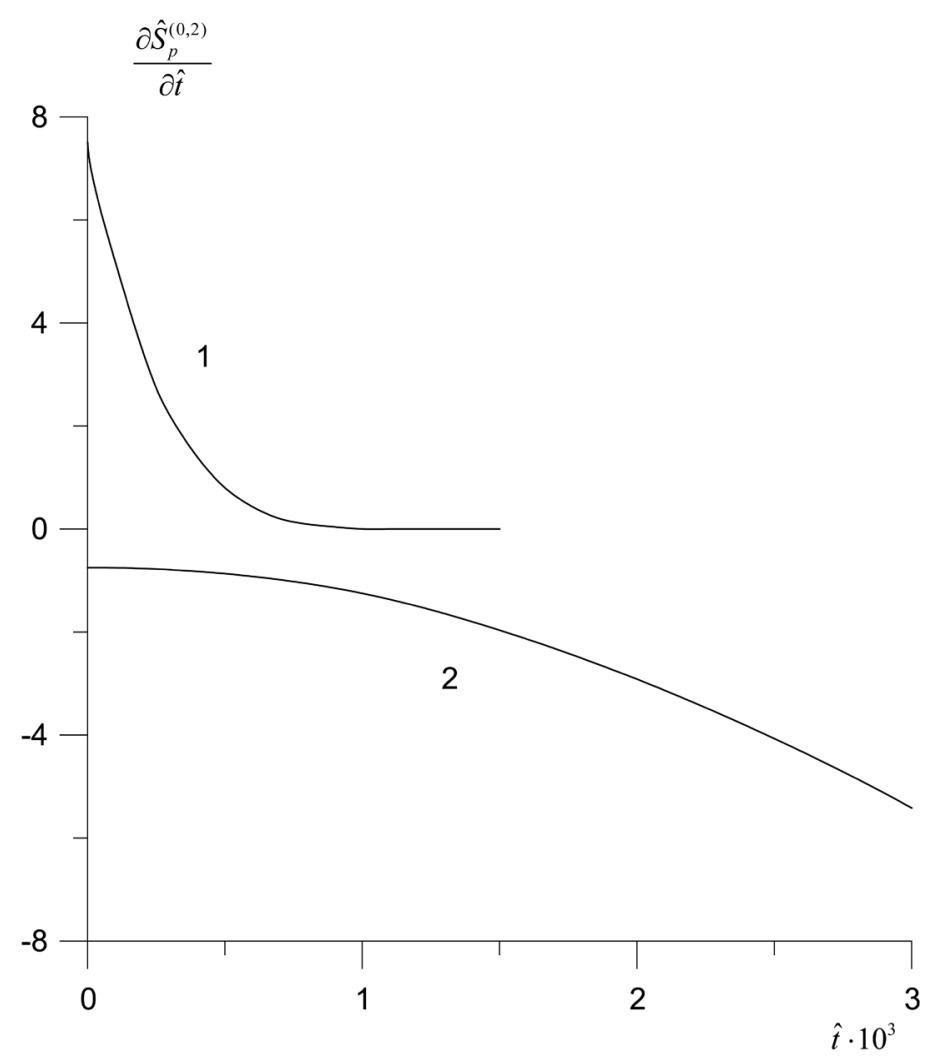

Figure 6. Time history of the pair entropy derivative $\partial \hat{S}_{p}^{(0,2)}(t) / \partial \hat{t}=\partial \delta \hat{S}_{p}^{R(0,2)}(t) / \partial \hat{t}$.

Curve 1 corresponds to $\left(\partial \delta \hat{S}_{p}^{R(0,2)}(t) / \partial \hat{t}\right) \cdot 10^{4}$ at a subcritical Re value of $\operatorname{Re}=129$.

Curve 2 corresponds to $\left(\partial \delta \hat{S}_{p}^{R(0,2)}(t) / \partial \hat{t}\right) \cdot 10^{3}$ at a supercritical Re value of $\operatorname{Re}=130$.

monotonically up to point $t=t_{*}$ where the solution breaks, and the entropy of the system $S_{p}^{(0)}(t)$ deviates increasingly farther from its stationary value $\bar{S}_{p}^{(0)}$ with increasing $t$. As revealed by calculations, fluctuations always fade out at $\operatorname{Re}<\operatorname{Re}_{0}^{*}$, and build up at $\operatorname{Re}>\operatorname{Re}_{0}^{*}$. The $\delta S_{p}^{(0,2)}(t)$ function is given by Equation (6.6).

From balance Equation (6.1) follows that at $R e<\mathrm{Re}_{0}^{*}$, the pair entropy formed in the system per unit time due to binary collisions,

$$
\Delta_{I N} S_{p}^{(0)}(t)=\Delta_{I N}\left(\bar{S}_{p}^{(0,1)}+\delta S_{p}^{R(0,1)}(t)\right)+\Delta_{I N}\left(\bar{S}_{p}^{(0,2)}+\delta S_{p}^{R(0,2)}(t)\right)
$$

exceeds the pair entropy removed through the surface confining the system per unit time,

$$
\Delta_{E X} S_{p}^{(0)}(t)=\Delta_{E X}\left(\bar{S}_{p}^{(0,1)}+\delta S_{p}^{R(0,1)}(t)\right)+\Delta_{E X}\left(\bar{S}_{p}^{(0,2)}+\delta S_{p}^{R(0,2)}(t)+\delta S_{p}^{S(0,2)}(t)\right)
$$

with the result that fluctuations produced by the system die out. At $\operatorname{Re}>\operatorname{Re}_{0}^{*}$, conversely, the pair entropy outflow $\Delta_{E X} S_{p}^{(0)}(t)$ exceeds its production $\Delta_{I N} S_{p}^{(0)}(t)$, and fluctuations build up. Thus, the case of instability onset in a flow around a sphere is a prevalence of pair entropy outflow over its production at the instant the Reynolds number reaches its critical value.

As revealed by calculations [19], the $S_{1}^{(0)}(t)$ Boltzmann entropy behaves exactly as the $S_{p}^{(0)}(t)$ pair entropy does, when the system passes through $\mathrm{Re}_{0}^{*}$. Hence, the cause of instability onset in the system, regardless of whether it is formulated in terms of pair entropy or in terms of Boltzmann entropy, remains the same.

Earlier in the analysis we restricted consideration exclusively to entropy fluctuations with $\delta S_{p}^{(0,2)}(t)<0$. The reason for this "asymmetry" is as follows. Under the second principle of thermodynamics, the probability of $\delta S_{1}^{(\alpha)}(t)<0 \quad\left(\delta S_{p}^{(\alpha)}(t)<0\right)$ fluctuations in an isolated system is so much greater than that of $\delta S_{1}^{(\alpha)}(t)>0$ 
$\left(\delta S_{p}^{(\alpha)}(t)>0\right)$ fluctuations that the latter rarely, if ever, occur in nature [16]. As revealed by study [19], entropy fluctuations with $\delta S_{1}^{(\alpha)}(t)>0 \quad\left(\delta S_{p}^{(0,2)}(t)>0\right)$ direct the system along extremely unlikely, i.e., impracticable path. Nevertheless, the probability of $\delta S_{1}^{(\alpha)}(t)>0 \quad\left(\delta S_{p}^{(\alpha)}(t)>0\right)$ fluctuations in an open system is not to be ruled out [19].

Evolution of fluctuations generated by system depends on two factors, on entropy production and removal through the surface confining the system. The fact that these factors were analyzed without resorting to any kinds of approximations encourages us to believe in the universal nature of the established cause of instability onset. Therefore, the principle according to which an open system retains (or loses) its stability can be formulated as follows.

An open system with time-independent boundary conditions has a stable stationary $\alpha$-state with entropy $\bar{S}_{p}^{(\alpha)}$ while entropy production in it exceeds entropy outflow through the surface confining the system for $\delta S_{p}^{(\alpha)}(t) \leq 0$ and does not exceed entropy outflow for $\delta S_{p}^{(\alpha)}(t) \geq 0$

$$
\begin{aligned}
& {\left[\Delta_{I N} S_{p}^{(\alpha)}(t)-\Delta_{E X} S_{p}^{(\alpha)}(t)\right] \geq 0, \quad t \geq 0, \text { for } \delta S_{p}^{(\alpha)}(t) \leq 0} \\
& {\left[\Delta_{I N} S_{p}^{(\alpha)}(t)-\Delta_{E X} S_{p}^{(\alpha)}(t)\right] \leq 0, \quad t \geq 0, \text { for } \delta S_{p}^{(\alpha)}(t) \geq 0}
\end{aligned}
$$

As soon as the parameters characterizing the system reach the values, at which at least one of inequalities (6.10a) and (6.10b) fails, the stationary $\alpha$-state of the open system becomes unstable.

The principle originally formulated for open system with time-independent boundary conditions can be expected to the case of open systems with time-dependent boundary conditions. Generally entropy $\bar{S}_{p}^{(\alpha)}(t)$ corresponding to an ensemble of systems may not be reckoned as stationary value. If so, Equation (6.1) no longer implies that the entropy production $\Delta_{I N} S_{p}^{(\alpha)}(t)$ equals its outflow $\Delta_{E X} S_{p}^{(\alpha)}(t)$. That is why, generally, the stability principle is formulated in terms of excess of the entropy production $\Delta_{I N} \delta S_{p}^{(\alpha)}(t)$ and excess of the entropy outflow $\Delta_{E X} \delta S_{p}^{(\alpha)}(t)$.

The $\alpha$-state with entropy $\bar{S}_{p}^{(\alpha)}(t)$ of an open system remains stable while the excess of entropy production generated in the system exceeds its excess of outflow through the surface confining the system for $\delta S_{p}^{(\alpha)}(t) \leq 0$ and does not exceed the excess of outflow for $\delta S_{p}^{(\alpha)}(t) \geq 0$

$$
\begin{aligned}
& {\left[\Delta_{I N} \delta S_{p}^{(\alpha)}(t)-\Delta_{E X} \delta S_{p}^{(\alpha)}(t)\right] \geq 0, \quad t>0, \text { for } \delta S_{p}^{(\alpha)}(t) \leq 0} \\
& {\left[\Delta_{I N} \delta S_{p}^{(\alpha)}(t)-\Delta_{E X} \delta S_{p}^{(\alpha)}(t)\right] \leq 0, \quad t>0, \text { for } \delta S_{p}^{(\alpha)}(t) \geq 0}
\end{aligned}
$$

As soon as the parameters characterizing the system reach the values, at which at least one of inequalities (6.11a) and (6.11b) fails, the $\alpha$-state of the open system becomes unstable.

Inequalities (6.10) for systems with time-independent boundary conditions are reduced to inequalities (6.11). However, the stability principle for stationary states (6.10) seems to be more "transparent". The above formulated stability principle remains invariant in going from pair to Boltzmann entropy [19].

\section{Interpretation of System Evolution in Terms of Pair Entropy}

In accordance with the principle of retention and loss of stability (6.11), in an open unstable system, any entropy fluctuation $\delta S_{p}^{(\alpha)}(t=0)<0$ begins to grow. In particular, for a system with time-independent boundary conditions

$$
\frac{\partial \delta S_{p}^{(\alpha)}(t)}{\partial t}=\frac{\partial S_{p}^{(\alpha)}(t)}{\partial t}<0 \quad \text { for } \delta S_{p}^{(\alpha)}(t=0)<0
$$

Based on the expression (7.1), we can formulate the criterion of evolution of an open system with lost stability.

An open unstable system with time-independent boundary conditions, takes a direction of evolution that provides the most rapid decrease in entropy. Namely, of the two directions of development of the instability, having the same values of the entropy and entropy derivative at the time $t=t_{0}$, fluctuations find such a direction that is characterized by lower value of the second derivative of entropy: 


$$
\left.\frac{\partial^{2} S_{p}^{(\alpha)}(t)}{\partial t^{2}}\right|_{t=t_{0}}<\left.\frac{\partial^{2} S_{p}^{(\beta)}(t)}{\partial t^{2}}\right|_{t=t_{0}}
$$

wherein

$$
\begin{gathered}
\left.S_{p}^{(\alpha)}(t)\right|_{t=t_{0}}=\left.S_{p}^{(\beta)}(t)\right|_{t=t_{0}},\left.\quad \frac{\partial S_{p}^{(\alpha)}(t)}{\partial t}\right|_{t=t_{0}}=\left.\frac{\partial S_{p}^{(\beta)}(t)}{\partial t}\right|_{t=t_{0}} \\
\text { for } \delta S_{p}^{(\alpha)}(t=0)<0 \quad \delta S_{p}^{(\beta)}(t=0)<0
\end{gathered}
$$

That is, at the time $t=t_{0}>0$, the system takes the $\alpha$-direction, for which the second derivative of the entropy with respect to time has a lower value compared to the respectively derivative for the $\beta$-direction.

In constructing the approximate solution to the equation for the pair function $f_{p}(t, \mathbf{x}, \mathbf{G}, v)$, only a limited number of terms is retain in the series of products of trajectory invariants (2.5). Different approximate solutions compatible with the boundary conditions of the problem differ in the number of terms retained in expression (2.5). To select the optimal approximate solutions, it is necessary to introduce an additional criterion. The logic of selecting one of the set of approximate solutions can be seen in the formulation of the criterion of evolution (7.2).

Let $\lambda$ be the set of parameters characterizing the $j$ th stable stationary solution $\hat{\bar{C}}_{i}^{(j)}=\hat{\bar{C}}_{i}^{(j)}(\lambda), j=1, \cdots, n$, $i=1, \cdots, s$, to the multimoment hydrodynamic equations, and let an increase in $\lambda$ be accompanied by the departure of the system state from the state of statistical equilibrium. In the simplest case, the entropy $\bar{S}_{p}^{(j)}(\lambda)$ is calculated in the entire space from one $j$ th solution. Suppose that, at some value of the parameter $\lambda=\lambda_{0}$, the pair entropy $\bar{S}_{p}^{(j)}(\lambda)$, calculated from the solution $\hat{\bar{C}}_{i}^{(j)}(\lambda), \quad j=1, \cdots, n, \quad i=1, \cdots, s$, occurs in a small vicinity $\Delta(\Delta>0)$ of the pair entropy $\bar{S}_{p}^{(0)}(\lambda)$. The entropy $\bar{S}_{p}^{(0)}(\lambda)$ is calculated based on the solution to the classic hydrodynamics equations, which are valid in the $\lambda \rightarrow 0$ limit. Based on expression (7.2), let us formulate a criterion for selecting the approximate stable solution for open system.

In interpreting the behavior of open system with time-independent boundary conditions, a solution that provides the fastest drop in entropy should be chosen from the set of stable approximate solutions to the multimoment hydrodynamics equations. Namely, at a certain value of the $\lambda=\lambda_{0}$ parameter, an approximate solution with the lowest value of the entropy derivative should be chosen among a few approximate solutions with the same entropy values in a small vicinity $\Delta$ of the pair entropy $\bar{S}_{p}^{(0)}(\lambda)$ :

$$
\begin{gathered}
\left.\frac{\partial \bar{S}_{p}^{(k)}(\lambda)}{\partial \lambda}\right|_{\lambda=\lambda_{0}}<\left.\frac{\partial \bar{S}_{p}^{(j)}(\lambda)}{\partial \lambda}\right|_{\lambda=\lambda_{0}} \quad j=1,2, \cdots, k-1, k+1, \cdots, n \\
\left|\bar{S}_{p}^{(0)}(\lambda)-\bar{S}_{p}^{(j)}(\lambda)\right|_{\lambda=\lambda_{0}} \mid<\Delta \quad j=1,2, \cdots, n
\end{gathered}
$$

This means that, at the given $\lambda=\lambda_{0}$, the priority lies with the $k$ th approximate stable solution to the multimoment hydrodynamics equations for which the derivative of the pair entropy with respect to $\lambda$ has the lowest value among the respective derivatives provided by other similar solutions. In more complex cases, it may be necessary to compare the second derivatives of the $\bar{S}_{p}(\lambda)$ entropy, as is done in (7.2).

Criteria (7.2), (7.3) are suitable for interpreting open systems with time-dependent boundary conditions.

\section{Vortex Shedding Regimes}

According to the $\mathrm{Sol}_{0}$ solution, the recirculating zone is formed in the wake behind a sphere. After the attainment of $\mathrm{Re}=\mathrm{Re}_{0}^{*}$, the periphery of the recirculating zone begins to pulsate periodically. Pulsating periphery demonstrates the absence of slightest indications of detachment from the core of the recirculating zone. Consequently, there is no vortex street in the far wake behind the sphere. Therefore, the $\mathrm{Sol}_{0}$ solution does not describe the vortex shedding.

Let the distribution of the particle-density flux be:

$$
\overparen{n U_{R}}=\operatorname{Re} \hat{C}_{20}\left(3-30 \cos ^{2} \Theta+35 \cos ^{4} \Theta\right) \frac{a^{2}}{R^{2}}\left(1-\frac{a^{2}}{R^{2}}\right)^{2}
$$




$$
\widetilde{n U_{\Theta}}=\operatorname{Re} \hat{C}_{20} \sin \Theta\left(12 \cos \Theta-28 \cos ^{3} \Theta\right) \frac{a^{4}}{R^{4}}\left(1-\frac{a^{2}}{R^{2}}\right)
$$

To obtain distribution (8.1), the second term on the right side of Equation (4.8) is written in $R$, $\Theta$-variables. Distribution (8.1) defines a vortex ring at $r_{0}$ distance from the sphere center, Figure 3(b). The vortex ring center is located at the $Z^{\prime}$ axis, which forms the $\delta$-angle with the $Z$-axis, Figure 7 . The inflowing gas velocity $\mathbf{U}_{0}$ is aligned with the positive direction of the $Z$ axis of reference frame $X Y Z$. The $X Y Z$ frame has its origin at the center of the sphere.

Let us retain all the terms proportional to $\hat{C}_{1}, \cdots, \hat{C}_{19}$ in the distributions of principal hydrodynamic values (4.4-4.10). These distributions have the form of linear combinations of the products of the inverse power functions of $r$ and trigonometric functions of the polar angle $\theta$. To bring distribution (8.1) to this form, we change in Equation (8.1) from the $R, \Theta$ variables to the $r^{\prime}, \theta^{\prime}$ variables, expand these expressions in a Taylor series in powers of $r_{0} / r^{\prime}, r^{\prime}>r_{0}$, and retain in this series only the terms independent of and linear in $r_{0} / r^{\prime}$ :

$$
\begin{aligned}
& \overparen{n U_{r^{\prime}}}=\overparen{n U_{\mathrm{R}}}-\overparen{n U_{\Theta}} \hat{C}_{21} \sin \theta^{\prime} \frac{a}{r^{\prime}} \\
& \overparen{n U_{\theta^{\prime}}}=\overparen{n U_{\Theta}}+\overparen{n U_{\mathrm{R}}} \hat{C}_{21} \sin \theta^{\prime} \frac{a}{r^{\prime}}
\end{aligned}
$$

Then, we change from the spherical coordinates $r^{\prime}, \theta^{\prime}, \varphi^{\prime}$ to the spherical coordinates $r, \theta, \varphi$. The variables $\theta, \varphi$ and $\theta^{\prime}, \varphi^{\prime}$ are, respectively, the polar and azimuthal angles of the vector $\mathbf{r}$ in the Cartesian reference frame with the $Z$ axis defining the direction of the flow incoming on the sphere and in the Cartesian reference frame with the $Z^{\prime}$ axis forming an angle $\delta$ with the $Z$ axis, Figure 7. The transformations performed give rise to azimuthal angle $\varphi$ dependent terms in the distributions of the particle-density flux $\overparen{n \mathbf{U}}$ :

$$
\overparen{n U_{\mathrm{r}}}=\overparen{n U_{r^{\prime}}} \quad \overparen{n U_{\theta}}=\overparen{n U_{\theta^{\prime}}} \cos \eta \quad \overparen{n U_{\varphi}}=-\overparen{n U_{\theta^{\prime}}} \sin \eta
$$

Here,

$$
\begin{gathered}
\sin \theta^{\prime} \sin \eta=\sin \varphi \sin \delta \\
\sin \theta^{\prime} \cos \eta=\cos \theta \cos \varphi \sin \delta+\sin \theta \cos \delta
\end{gathered}
$$

Along with the coefficient $\hat{C}_{20}$, the transformed expression (8.3) for the distribution of the particle-density flux $\overparen{n \mathbf{U}}$ contains two additional coefficients: $\hat{C}_{21}=r_{0} / a$ and $\hat{C}_{22}=\cos \delta$. The coefficient $\hat{C}_{21}$ characterizes the distance from the vortex ring to the sphere surface, whereas the coefficient $\hat{C}_{22}$ characterizes the deviation of the vortex ring center from the $Z$ axis. Trajectory invariants for expansion (4.2), which lead to the distribution (8.3), are not written in the present Section.

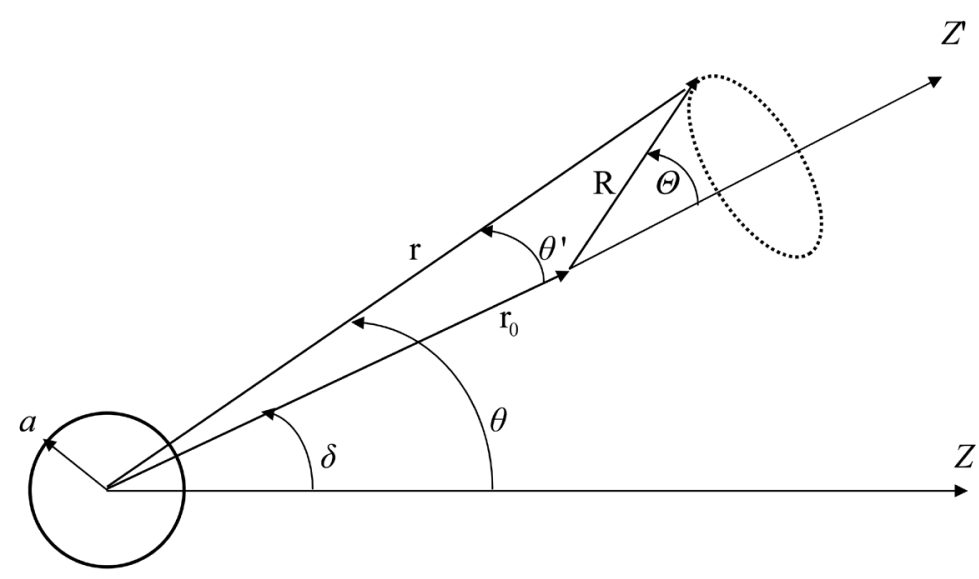

Figure 7. The $Z$ axis of the $X Y Z$ frame with its origin at the center of the sphere; $r, \theta$, and $\varphi$ are the spherical coordinates of some point $\mathbf{x}$. 
The distributions of the principal hydrodynamic values (4.4)-(4.10) associated with the coefficients $\hat{C}_{1}, \cdots, \hat{C}_{19}$ should be supplemented with distribution (8.3). The presence of $\varphi$-dependent terms in Equation (8.3) for the distribution of the particle-density flux $\overparen{n \mathbf{U}}$ leads to the appearance of these terms in the hydrodynamics equations. Taking into account the dependence on $\varphi$ is beyond the accuracy limits of the approximation under consideration.

Expand expression (8.3) for the distribution of the particle-density flux $\overparen{n \mathbf{U}}$ in a Fourier series in the azimuthal angle $\varphi$ and substitute the zeroth-order Fourier expansion term in the multimoment hydrodynamics Equations (2.9)-(2.11). Eighteen nonlinear algebraic equations are presented by relationships (A8)-(A10) in [8]. Righthand sides of Equations (A8)-(A10) from [8] contain twenty coefficients $\hat{C}_{i}, i=1, \cdots, 20$. In accordance with the algorithm presented in [8], we supplement these equations by terms containing the coefficients $\hat{C}_{21}$ and $\hat{C}_{22}$. Let us supplement eighteen algebraic Equations (A8-A10) from [8] with three algebraic Equation (4.12) and differential Equation (4.2). As a result, we obtain a closed set of nonlinear equations of twenty-second order S22 for the coefficients $\hat{C}_{i}, \quad i=1, \cdots, 22$. It turned out that, in the investigated range of Re, of great many solutions to the system S22, only two solutions correspond to such an entropy value that allows these solutions to compete with the solution $\mathrm{Sol}_{0}$. We denote these solutions as $\mathrm{Sol}_{1}$ and $\mathrm{Sol}_{2}$.

In the rearrangement of the distribution (8.1), the terms nonlinear in $r_{0} / r^{\prime}$ were omitted. Calculations showed that the omitted terms have no significant influence on the solutions $\mathrm{Sol}_{1}$ and $\mathrm{Sol}_{2}$. Substitute the full Fourier expansion of expression (8.3) for the distribution of the particle-density flux $\overparen{n \mathbf{U}}$ into the multimoment hydrodynamics Equations (2.9)-(2.11) and integrate the resulting nonlinear system of differential equations over $\varphi$. In this case, we arrive at system S22 supplemented by several terms. Calculations have shown that these supplements produce no significant influence on the solutions $\mathrm{Sol}_{1}$ and $\mathrm{Sol}_{2}$.

\section{Selecting the Direction of Instability Development}

Figure 8 shows the time dependence of the $S_{p(0)}^{(0,2)}(t)$ dimensionless pair entropy (6.3) calculated from the so-

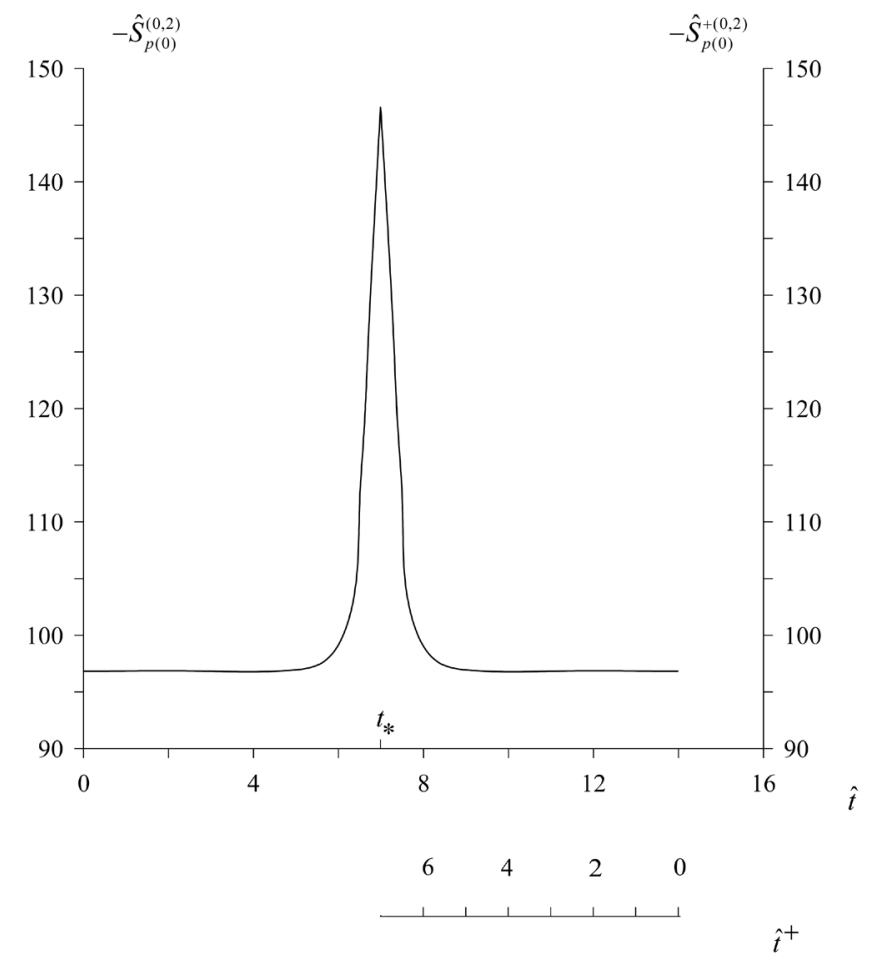

Figure 8. Time behavior of the pair entropy $\hat{S}_{p(0)}^{(0,2)}(t), \hat{S}_{p(0)}^{+(0)}\left(t^{+}\right)$ calculated within the layer $\mathrm{H}_{0}$ at $\hat{r}_{2}=\hat{r}_{2}^{(0)}=2.392, \mathrm{Re}=400$, $\hat{t}_{*}=6.99020$. 
lution $\mathrm{Sol}_{0}$ at $\mathrm{Re}=400$. The pair entropy is calculated in the dominant order, $S_{p(0)}^{(0,2)}(t)=k n_{0} v_{0} \mathrm{Ma}^{4} \hat{S}_{p(0)}^{(0,2)}(t)$. In calculating the $S_{p(0)}^{(0,2)}(t)$ pair entropy, the spatial integration (A.4) is performed over a semispherical concentric layer $\mathrm{H}_{0}$, Figure 9. The subscript in brackets corresponds to the limits of spatial integration. The $\mathrm{Sol}_{0}$ solu- $^{-}$ tion describes periodic pulsations of the recirculating zone in the wake behind the sphere. The movement of the representative point over the curve (Figure 8) from $t=0$ to $t=t_{*}$ corresponds to the expansion or, in other words, the excitation of the recirculating zone, $t=\left(\operatorname{Re} a / 2 U_{0}\right) \hat{t}$. The pair entropy $S_{p(0)}^{(0,2)}(t)$ will be used as a measure of excitation degree of recirculating zone. Beginning from the time $t=0$ up to the time $t=t_{*}$, the entropy decreases permanently. This behavior of the entropy corresponds to the movement away of the system state that lost its stability from the state of statistical equilibrium. By the time $t=t_{*}$, the degree of excitation of the recirculating zone reaches a maximum, which corresponds to the minimum value of the $S_{p(0)}^{(0,2)}(t)$ function. At the lettime $t=t_{*}$, the $\mathrm{Sol}_{0}$ solution to the multimoment hydrodynamics equations breaks. The movement of the representative point over the curve (Figure 8) from $t=t_{*}$ to $t=2 t_{*}$ corresponds to the return of the most excited recirculating zone to its original position, i.e., the position corresponding to the time $t=0$. Since the time $t=t_{*}$, the movement of the representative point is described by the reverse equations of multimoment hydrodynamics [6]. The reverse equations are solved with regressive timing along the time axis, from the past to the future. This timing order is represented on the axis beneath the abscissa in Figure $3\left(t^{+}=-t+2 t_{*}\right.$ within $\left.t_{*} \leq t \leq 2 t_{*}\right)$. The $S_{p(0)}^{(0,2)}(t)$ pair entropy exists in the range $0 \leq t \leq t_{*}$, whereas the $S_{p(0)}^{+(0,2)}\left(t^{+}\right)$pair entropy (A.15), within $t_{*} \leq t \leq 2 t_{*}$. The distribution of the hydrodynamics values involved in the expressions for $S_{p(0)}^{(0,2)}(t)$ and $S_{p(0)}^{+(0,2)}\left(t^{+}\right)$are conjugated with the coefficients $\hat{C}_{i}^{(0)}(t)$ and $\hat{C}_{i}^{+(0)}\left(t^{+}\right), \quad i=1, \cdots, 20$. Relationships (5.4) between the coefficients $\hat{C}_{i}^{+(0)}\left(t^{+}\right)$and $\hat{C}_{i}^{(0)}(t)$ yield $\left.S_{p(0)}^{+(0,2)}\left(t^{+}\right)\right|_{t^{+}=t_{0}}=\left.S_{p(0)}^{(0,2)}(t)\right|_{t=t_{0}}$ for any time $t_{0}$ within, $0 \leq t_{0} \leq t_{*}$. Figure 8 shows that most of the pulsation half-period $t_{*}$, approximately $5 t_{*} / 7$, the entropy of the system decreases rather weakly. A sharp decrease in the entropy $S_{p(0)}^{(0,2)}(t)$ occurs within a short time of $2 t_{*} / 7$. The function $\tilde{S}_{p(0)}^{(0,2)}\left(t-t^{(0)}\right)$ is a portion of the pair entropy $S_{p(0)}^{(0,2)}(t)$, which specifies the behavior of the entropy in the neighborhood, $t_{*}-t^{(0)}$, of the point of solution break, $t=t_{*}$

$$
S_{p(0)}^{(0,2)}(t)=\tilde{S}_{p(0)}^{(0,2)}\left(t-t^{(0)}\right)
$$

Represent the $\tilde{S}_{p(0)}^{(0,2)}\left(t-t^{(0)}\right)$ function as a sum of functions:

$$
\tilde{S}_{p(0)}^{(0,2)}\left(t-t^{(0)}\right)=\tilde{S}_{p(1)}^{(0,2)}\left(t-t^{(0)}\right)+\tilde{S}_{p(2)}^{(0,2)}\left(t-t^{(0)}\right)
$$

The $\tilde{S}_{p(1)}^{(0,2)}\left(t-t^{(0)}\right)$ entropy is calculated within a semispherical concentric layer $H_{1}$ (Figure 9). The

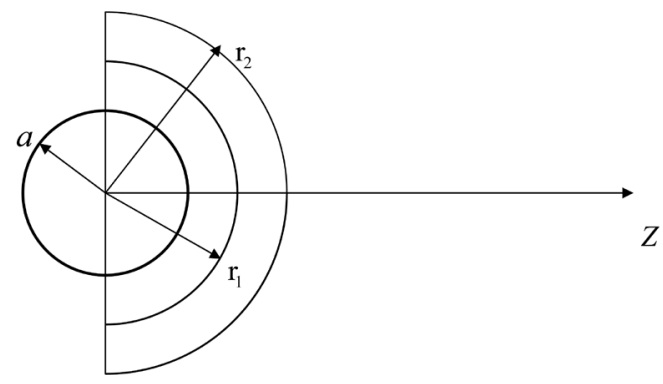

\footnotetext{
Figure 9. Semispherical concentric layer $\mathrm{H}_{0}: 1 \leq r \leq r_{2}, \frac{\pi}{2} \leq \theta \leq 0,2 \pi \leq \varphi \leq 0$; semispherical concentric layer $\mathrm{H}_{1}: 1 \leq r \leq r_{1}, \frac{\pi}{2} \leq \theta \leq 0,2 \pi \leq \varphi \leq 0$; semispherical concentric layer $\mathrm{H}_{2}: r_{1} \leq r \leq r_{2}, \frac{\pi}{2} \leq \theta \leq 0,2 \pi \leq \varphi \leq 0$.
} 
$\tilde{S}_{p(2)}^{(0,2)}\left(t-t^{(0)}\right)$ entropy is calculated within a semispherical concentric layer $\mathrm{H}_{2}$. In calculating $\tilde{S}_{p(1)}^{(0,2)}\left(t-t^{(0)}\right)$ and $\tilde{S}_{p(2)}^{(0,2)}\left(t-t^{(0)}\right)$, the parameters $r_{1}=r_{1}^{(0)}$ and $r_{2}=r_{2}^{(0)}$ were set constant.

Let the representative point move upwards along the curve in Figure 8. By the time $t=t_{1}$, the recirculating zone reaches a degree of excitation characterized by the $S_{p(0)}^{(0,2)}\left(t_{1}\right)$ function. The time $t=t_{1}$ corresponds to the point of intersection of the curves 1 and 2 in Figure 10. At the time $t=t_{1}$, the relations

$$
\begin{gathered}
\left.\tilde{S}_{p(0)}^{(0,2)}\left(t-t^{(0)}\right)\right|_{t=t_{1}}=\left.\breve{S}_{p(1)}^{(0,2)}\left(t-t^{(0)}\right)\right|_{t=t_{1}}+\left.\breve{S}_{p(2)}^{(i, 2)}\left(t-t^{(0)}\right)\right|_{t=t_{1}} \quad i=1,2 \\
\left.\frac{\partial \tilde{S}_{p(0)}^{(0,2)}\left(t-t^{(0)}\right)}{\partial t}\right|_{t=t_{1}}=\left.\frac{\partial \breve{S}_{p(1)}^{(0,2)}\left(t-t^{(0)}\right)}{\partial t}\right|_{t=t_{1}}+\left.\frac{\partial \breve{S}_{p(2)}^{(i, 2)}\left(t-t^{(0)}\right)}{\partial t}\right|_{t=t_{1}} \quad i=1,2
\end{gathered}
$$

are valid.

The left-hand side of Equation (9.3a) defines the $\tilde{S}_{p(0)}^{(0,2)}\left(t-t^{(0)}\right)$ function calculated within the layer $\mathrm{H}_{0}$. The $\breve{S}_{p(1)}^{(0,2)}\left(t-t^{(0)}\right)$ function on the right-hand side of (9.3a) is calculated within the layer $\mathrm{H}_{1}$. In accordance with definition

$$
\breve{S}_{p(i)}^{(0,2)}\left(t-t^{(0)}\right)=\tilde{S}_{p(i)}^{(0,2)}\left(t-t^{(0)}+\Delta t\right) \quad i=0,1 \quad \Delta t>0
$$

The $\breve{S}_{p(1)}^{(0,2)}\left(t-t^{(0)}\right)$ function on the right-hand side of Equation (9.3a) was calculated within the $\mathrm{H}_{2}$ layer from the solution $\mathrm{Sol}_{1}$ at $\mathrm{Re}=400$. The $\mathrm{Sol}_{1}$ solution describes the motion of a single vortex structure in the wake behind the sphere. The coefficient $\hat{C}_{21}=r_{0} / a$ in the Sol $_{1}$ solution highly accurately defines the position of the vortex structure at the $Z$ axis, since $\hat{C}_{22}=\cos \delta \cong 1$ (Figure 7). In accordance with the Sol $_{1}$ solution, the vortex structure monotonically recedes from the surface of the sphere, being accompanied by dissipation of the vortex structure. This behavior of the vortex structure causes the increase in the $\breve{S}_{p(2)}^{(1,2)}\left(t-t^{(0)}\right)$ pair entropy as $t$ grows. In calculating $\breve{S}_{p(2)}^{(1,2)}\left(t-t^{(0)}\right)$, the initial time for the movement of a single vortex structure, $t^{(1)}$, is chosen so that, at the time $t=t_{1}-t^{(0)}$, the vortex structure moving in space would reach the position

$$
\hat{\tilde{C}}_{21}\left(t_{1}-t^{(0)}\right)=\hat{r}_{1}^{(0)}
$$

where $\hat{\tilde{C}}_{21}\left(t-t^{(0)}\right)=\hat{C}_{21}\left(t-t^{(0)}-t^{(1)}\right)$. That is, at the time $t=t_{1}$, the position of a single vortex structure at the $\mathrm{Z}$ axis is determined by $r_{1}^{(0)}$, which defines the inner boundary of the $\mathrm{H}_{2}$ layer.

The left-hand side of Equation (9.3) corresponds to the state of the system $S^{-}$at which the $\mathrm{H}_{0}$ layer is completely occupied by the recirculating zone, given by the $\mathrm{Sol}_{0}$ solution. By the time $t=t_{1}$, the recirculating zone reaches the degree of excitation that is characterized by the $\tilde{S}_{p(0)}^{(0,2)}\left(t_{1}-t^{(0)}\right)$ function. According to Equation (9.2), in the $S^{-}$state, the core of the recirculating zone has a degree of excitation characterized by the function $\tilde{S}_{p(1)}^{(0,2)}\left(t_{1}-t^{(0)}\right)$, whereas the periphery recirculating zone has a degree of excitation characterized by the function $\tilde{S}_{p(2)}^{(0,2)}\left(t_{1}-t^{(0)}\right)$. The right-hand side of Equation (9.3) corresponds to a qualitatively different state of the system $S^{+}$. Namely, in the $S^{+}$state, the periphery of the recirculating zone with the degree of excitation $\tilde{S}_{p(2)}^{(0,2)}\left(t_{1}-t^{(0)}\right)$, corresponding to the state of the system $S^{-}$, is replaced by a single vortex structure in the $\mathrm{H}_{2}$ layer, described by the $\mathrm{Sol}_{1}$ solution. This vortex structure is characterized by the $\breve{S}_{p(2)}^{(1,2)}\left(t-t^{(0)}\right)$ entropy. In the $S^{+}$state, the core of recirculating zone with the degree of excitation $\tilde{S}_{p(1)}^{(0,2)}\left(t_{1}-t^{(0)}\right)$ corresponding to the state of the system $S^{-}$, is replaced in the $\mathrm{H}_{1}$ layer by a recirculating zone core with the different degree of 


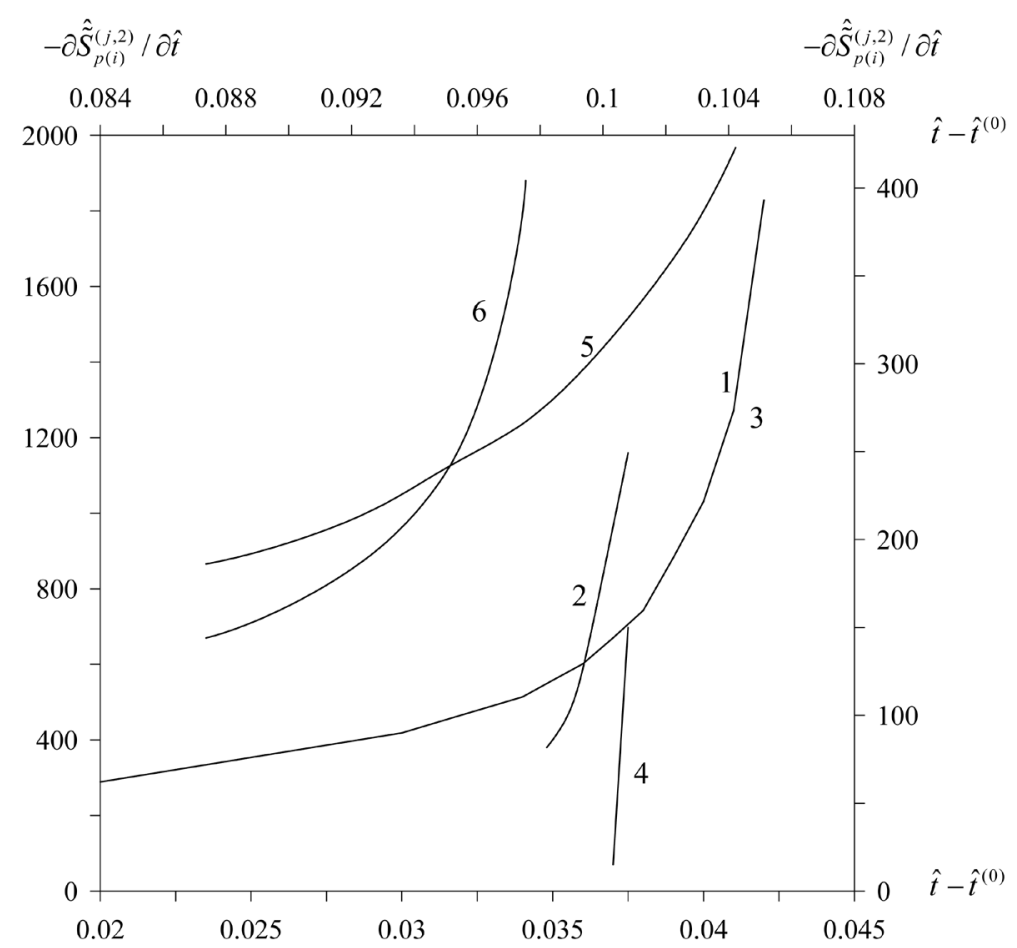

Figure 10. Time dependence of the entropy derivative $\partial \hat{\tilde{S}}_{p(i)}^{(j, 2)}\left(t-t^{(0)}\right) / \partial \hat{t}, i=0,3, j=0,1,2$. The function $\partial \hat{\tilde{S}}_{p(0)}^{(0,2)}\left(t-t^{(0)}\right) / \partial \hat{t}$ calculated within the layer $\mathrm{H}_{0}$ at $\hat{r}_{2}=\hat{r}_{2}^{(0)}=2.392$, Re $=400, \hat{t}^{(0)}=6.94658$ is represented by curve 1. Curve 2 is $\partial \hat{\tilde{S}}_{p(3)}^{(1)}\left(t-t^{(0)}\right) / \partial \hat{t}=\partial\left[\hat{S}_{p(1)}^{(0)}\left(t-t^{(0)}\right)+\hat{\tilde{S}}_{p(2)}^{(1)}\left(t-t^{(0)}\right)\right] / \partial \hat{t} \quad$ at $\operatorname{Re}=400, \hat{t}^{(0)}=6.94658$. The function $\partial \hat{\vec{S}}_{p(1)}^{(0,2)}\left(t-t^{(0)}\right) / \partial \hat{t}$ is calculated in the layer $\mathrm{H}_{1}$ at $\hat{r}_{1}=\hat{r}_{1}^{(0)}=1.740$; the function $\partial \hat{S}_{p(2)}^{(1)}\left(t-t^{(0)}\right) / \partial \hat{t}$ is calculated in the layer $\mathrm{H}_{2}$ at $\hat{r}_{1}=\hat{r}_{1}^{(0)}=1.740, \hat{r}_{2}=\hat{r}_{2}^{(0)}=2.392$. The time of restructuring is $\hat{t}=\hat{t}_{1}=\hat{t}^{(0)}+0.03605$. The time count origin for the movement of a single vortex structure is $\hat{t}^{(1)}=0.03561$ for $\hat{t}_{1}=\hat{t}^{(0)}+0.03605$. The function $\partial \hat{\tilde{S}}_{p(0)}^{(0,2)}\left(t-t^{(0)}\right) / \partial \hat{t}$ calculated within the layer $\mathrm{H}_{0}$ at $\hat{r}_{2}=\hat{r}_{2}^{(0)}=2.204, \mathrm{Re}=$ 400, $\hat{t}^{(0)}=6.94658$ is represented by curve 3. Curve 4 is $\partial \hat{\tilde{S}}_{p(3)}^{(1,2)}\left(t-t^{(0)}\right) / \partial \hat{t}=\partial\left[\hat{S}_{p(1)}^{(0,2)}\left(t-t^{(0)}\right)+\hat{\tilde{S}}_{p(2)}^{(1,2)}\left(t-t^{(0)}\right)\right] / \partial \hat{t}$ at $\operatorname{Re}=400, \hat{t}^{(0)}=6.94658$. The function $\partial \hat{S}_{p(1)}^{(0,2)}\left(t-t^{(0)}\right) / \partial \hat{t}$ is calculated in the layer $H_{1}$ at $\hat{r}_{1}=\hat{r}_{1}^{(0)}=1.580$, whereas the function $\partial \hat{S}_{p(2)}^{(1,2)}\left(t-t^{(0)}\right) / \partial \hat{t}$ in the layer $\mathrm{H}_{2}$ at $\hat{r}_{1}=\hat{r}_{1}^{(0)}=1.580, \hat{r}_{2}=\hat{r}_{2}^{(0)}=2.204$. The restructuring time is $\hat{t}=\hat{t}_{1}=\hat{t}^{(0)}+0.03749$. The initial point of time count for the movement of a single vortex structure is $\hat{t}^{(1)}=0.03561$ at $\hat{t}_{1}=\hat{t}^{(0)}+0.03749$. The function $\partial \hat{\tilde{S}}_{p(0)}^{(0,2)}\left(t-t^{(0)}\right) / \partial \hat{t}$ calculated within the layer $\mathrm{H}_{0}$ at $\hat{r}_{2}=\hat{r}_{2}^{(0)}=4.162, \operatorname{Re}=260, \hat{t}^{(0)}=5.26473$ is represented by curve 5 . Curve 6 is $\partial \hat{\tilde{S}}_{p(3)}^{(2,2)}\left(t-t^{(0)}\right) / \partial \hat{t}=\partial\left[\hat{\hat{S}}_{p(1)}^{(0,2)}\left(t-t^{(0)}\right)+\hat{\vec{S}}_{p(2)}^{(2,2)}\left(t-t^{(0)}\right)\right] / \partial \hat{t} \quad$ at $\operatorname{Re}=260, \hat{t}^{(0)}=5.26473$. The function $\partial \hat{S}_{p(1)}^{(0,2)}\left(t-t^{(0)}\right) / \partial \hat{t} \quad$ calculated within the layer $\mathrm{H}_{1}$ at $\hat{r}_{1}=\hat{r}_{1}^{(0)}=1.672$; the function $\partial \hat{\vec{S}}_{p(2)}^{(2,2)}\left(t-t^{(0)}\right) / \partial \hat{t}$ is calculated in the layer $\mathrm{H}_{2}$ at $\hat{r}_{1}=\hat{r}_{1}^{(0)}=1.672, \hat{r}_{2}=\hat{r}_{2}^{(0)}=4.162$. The time of restructuring is $\hat{t}=\hat{t}_{1}=\hat{t}^{(0)}+0.09539$. The initial point of time count for the movement of a single vortex structure is $\hat{t}^{(1)}=0.09310$ at $\hat{t}_{1}=\hat{t}^{(0)}+0.09539$. For curves $1,2,3,4$, the abscissa is given at the bottom, and the ordinate, on the left. For curves 5,6 , the abscissa is given at the top, and the ordinate, on the right. 
excitation. The degree of excitation of the recirculating zone core in the $S^{+}$state is higher as compared to the degree of excitation of the recirculating zone in the $S^{-}$state

$$
\breve{S}_{p(1)}^{(0,2)}\left(t_{1}-t^{(0)}\right)=\tilde{S}_{p(1)}^{(0,2)}\left(t_{1}-t^{(0)}+\Delta t_{1}\right)<\tilde{S}_{p(1)}^{(0,2)}\left(t_{1}-t^{(0)}\right) \quad \Delta t_{1}>0
$$

Thus, expressions (9.3) describe the instantaneous rearrangement of the flow at the time $t=t_{1}$.

The curve 1 in Figure 10 specifies the time evolution of the $\partial \tilde{S}_{p(0)}^{(0,2)}\left(t-t^{(0)}\right) / \partial t$ entropy derivative which is calculated within the $\mathrm{H}_{0}$ layer. The curve 2 in Figure 10 specifies the time evolution of the derivative $\partial\left[\breve{S}_{p(1)}^{(0,2)}\left(t-t^{(0)}\right)+\breve{S}_{p(2)}^{(1,2)}\left(t-t^{(0)}\right)\right] / \partial t=\partial \tilde{S}_{p(3)}^{(1,2)}\left(t-t^{(0)}\right) / \partial t$. The $\partial \breve{S}_{p(1)}^{(0,2)}\left(t-t^{(0)}\right) / \partial t$ function is calculated in the $\mathrm{H}_{1}$ layer. The $\partial \breve{S}_{p(2)}^{(1,2)}\left(t-t^{(0)}\right) / \partial t$ function is calculated in the $\mathrm{H}_{2}$ layer. In calculating $\partial \breve{S}_{p(2)}^{(1,2)}\left(t-t^{(0)}\right) / \partial t$, the variable initial time $t^{(1)}$ for the movement of a single vortex structure is chosen at each moment of time $t$ so that, by the time $t-t^{(0)}$, the vortex structure moving in space would reach the position $\hat{\tilde{C}}_{21}\left(t-t^{(0)}\right)=\hat{r}_{1}^{(0)}$, where $r_{1}^{(0)}$ specifies the inner boundary of the $\mathrm{H}_{2}$ layer. For each moment of time $t$, not too close to the time of recovery $t_{*}$, there is a time interval, $\Delta t>0, t+\Delta t<t_{*}$, which ensures the validity of equality (9.3a). However, as follows from Figure 10, only at the time of restructuring $t=t_{1}$, the validity of Equation (9.3a) entails the validity of Equation (9.3b). Then, in accordance with the criterion (7.2), the system takes the direction of evolution set by the flow restructured at the time $t=t_{1}$. The state of the restructured flow is characterized by the terms on the right-hand side of Equation (9.3). Information required to build the curves in Figure 10 and Figure 11 is given in Figures 4-7 in [20].

The evolution of the reconstructed flow proceeds as follows. A single vortex structure located at the time $t=t_{1}$ in the $\mathrm{H}_{2}$ layer, begins to move downstream in strict accordance with the $\mathrm{Sol}_{1}$ solution. The inner boundary $r_{1}=\tilde{r}_{1}^{(1)}$ of the $\mathrm{H}_{2}$ layer, which is simultaneously the outer boundary of the $\mathrm{H}_{1}$ layer, is bound to the position of a single vortex structure: $\hat{\tilde{r}}_{1}^{(1)}\left(t-t^{(0)}\right)=\hat{\tilde{C}}_{21}\left(t-t^{(0)}\right)=\hat{C}_{21}\left(t-t^{(0)}-t^{(1)}\right)$; i.e., this boundary moves together with the vortex structure at a fixed $t^{(1)}$ corresponding to $t=t_{1}$. The time behavior of the $\breve{S}_{p(3)}^{(0,2)}\left(t-t^{(0)}-\Delta t_{1}\right)$ pair entropy is represented by the curve 3 in Figure 11. The $\breve{S}_{p(3)}^{(0,2)}\left(t-t^{(0)}-\Delta t_{1}\right)$ function is calculated from the $\mathrm{Sol}_{0}$ solution within the $\mathrm{H}_{1}$ layer with the moving outer boundary $\tilde{r}_{1}^{(1)}\left(t-t^{(0)}\right)$. At the restructuring time $t=t_{1}$, a single vortex structure is positioned at $\tilde{r}_{1}^{(1)}\left(t_{1}-t^{(0)}\right)=r_{1}^{(0)}$. By the time $t=t_{3}$, $t_{3}=2 t_{*}-t_{1}$, the vortex structure reaches the position $\hat{\tilde{C}}_{21}\left(t_{3}-t^{(0)}\right)$, in accordance with the Sol $_{1}$ solution. Defined by the Sol $\mathrm{S}_{1}$ solution, the outer boundary $\tilde{r}_{1}^{(1)}\left(t_{3}-t^{(0)}\right)$ of the $\mathrm{H}_{1}$ layer at the time $t=t_{3}$ sets the value of $r_{2}^{(0)}: \tilde{r}_{1}^{(1)}\left(t_{3}-t^{(0)}\right)=r_{2}^{(0)}$. Since the time of recovery $t=t_{*}$, the movement of the system is described by the reverse multimoment hydrodynamics equations [6], which enable to calculate the time dependence of the reverse pair entropy $\widehat{S}_{p(3)}^{+(0,2)}\left(t^{+}-t^{(0)}-\Delta t_{1}\right)$. The reverse equations are solved with regressive timing direction along the time axis, from the past to the future. This timing order, $t^{+}=-t+2 t_{*}$, is shown on the axis beneath the abscissa in Figure 11 in the range $t_{*} \leq t \leq 2 t_{*}$. The time $t=t_{3}$ for progressive timing corresponds to the time $t^{+}=t_{1}$ for the regressive timing. The curves 3 and 2 in Figure 11 are intersected at a time of $t=t_{3}$. Then,

$$
\begin{aligned}
& \left.\breve{S}_{p(3)}^{(0,2)}\left(t-t^{(0)}-\Delta t_{1}\right)\right|_{t=t_{1}}=\left.\breve{S}_{p(1)}^{(0,2)}\left(t-t^{(0)}-\Delta t_{1}\right)\right|_{t=t_{1}} \\
& \left.\breve{S}_{p(3)}^{+(0,2)}\left(t^{+}-t^{(0)}-\Delta t_{1}\right)\right|_{t^{+}=t_{1}}=\left.\breve{S}_{p(0)}^{+(0,2)}\left(t^{+}-t^{(0)}-\Delta t_{1}\right)\right|_{t^{+}=t_{1}}
\end{aligned}
$$

In Figure 11, the functions $\breve{S}_{p(1)}^{(0,2)}\left(t-t^{(0)}-\Delta t_{1}\right)$ and $\breve{S}_{p(0)}^{(0,2)}\left(t-t^{(0)}-\Delta t_{1}\right)$ are represented by curves 1 and 2. 


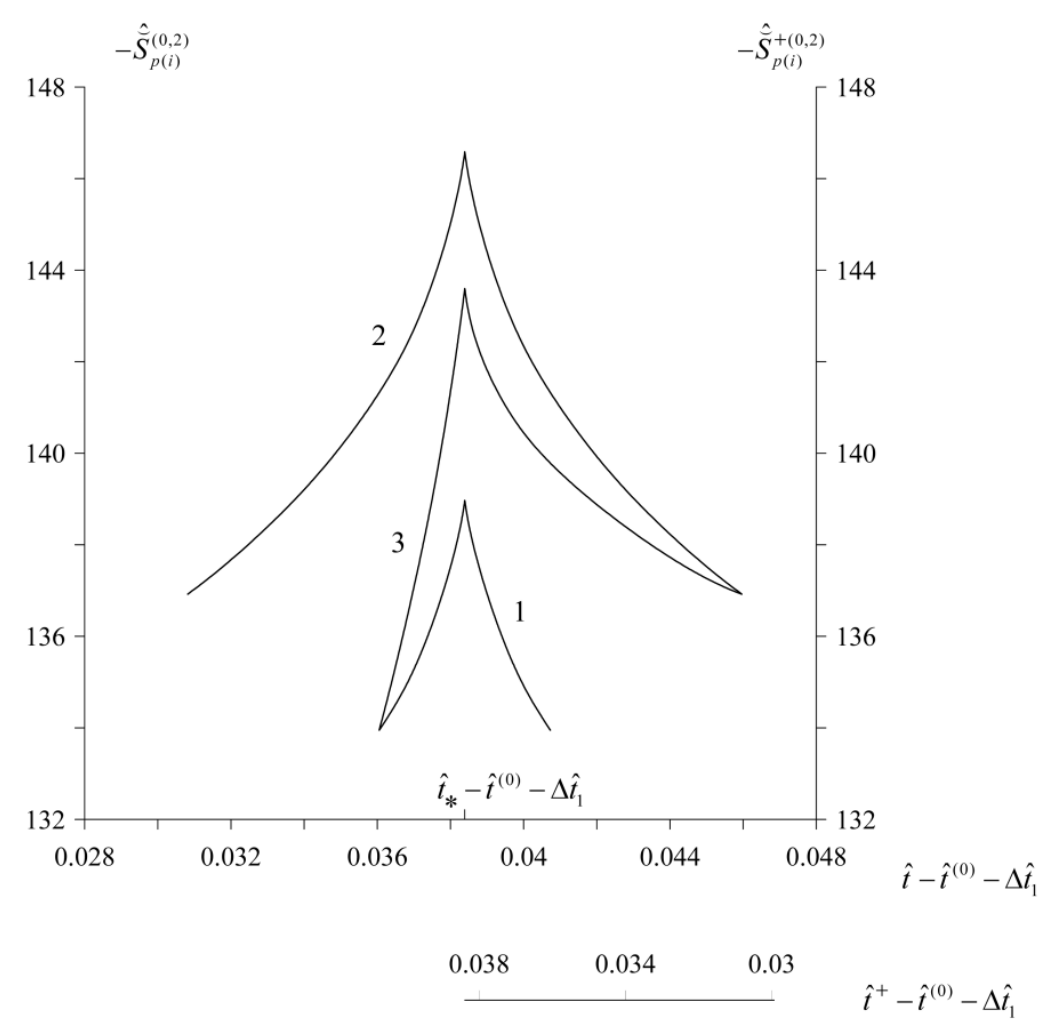

Figure 11. Time behavior of the pair entropy, $R e=400$. The restructuring time is $\hat{t}=\hat{t}_{1}=\hat{t}^{(0)}+0.03605$; the time of detachment is $\hat{t}=\hat{t}_{3}=\hat{t}^{(0)}+0.05119, \hat{t}^{(0)}=6.947$, $\Delta \hat{t}_{1}=0.00523$. The pair entropy $\hat{\vec{S}}_{p(i)}^{(0,2)}\left(t-t^{(0)}-\Delta t_{1}\right), i=0,1,3$, exist in the range $0 \leq t \leq t_{*}$. The pair entropy $\hat{\vec{S}}_{p(i)}^{+(0,2)}\left(t^{+}-t^{(0)}-\Delta t_{1}\right), i=0,1,3$, exist in the range $t_{*} \leq t \leq 2 t_{*}, t^{+}=-t+2 t_{*}$. Curve 1 represents the functions $\hat{\vec{S}}_{p(1)}^{(0,2)}\left(t-t^{(0)}-\Delta t_{1}\right)$ and $\hat{\vec{S}}_{p(1)}^{+(0,2)}\left(t^{+}-t^{(0)}-\Delta t_{1}\right)$ calculated within the layer $\mathrm{H}_{1}$ at $\hat{r}_{1}=\hat{r}_{1}^{(0)}=1.740$. Curve 2 represents the functions $\hat{\vec{S}}_{p(0)}^{(0,2)}\left(t-t^{(0)}-\Delta t_{1}\right)$ and $\hat{\vec{S}}_{p(0)}^{+(0,2)}\left(t^{+}-t^{(0)}-\Delta t_{1}\right)$ calculated within the layer $\mathrm{H}_{0}$ at $\hat{r}_{2}=\hat{r}_{2}^{(0)}=2.392$. Curve 3 represents the functions $\hat{\vec{S}}_{p(3)}^{(0,2)}\left(t-t^{(0)}-\Delta t_{1}\right)$ and $\hat{\vec{S}}_{p(3)}^{+(0,2)}\left(t^{+}-t^{(0)}-\Delta t_{1}\right)$ calculated within the layer $\mathrm{H}_{1}$. The movement of the outer boundary $r_{1}=\tilde{r}_{1}^{(1)}$ of the layer $\mathrm{H}_{1}$ is given by $\hat{\tilde{r}}_{1}^{(1)}\left(t=t_{1}-t(0)\right)=\hat{r}_{1}^{(0)}=1.740, \quad \hat{\tilde{r}}_{1}^{(1)}\left(t=t_{3}-t(0)\right)=\hat{r}_{2}^{(0)}=2.392$.

In accordance with Equations (9.4b) and (5.4), and the definition of the pair entropy, at the time $t=t_{3}$ (equivalent to $t^{+}=t_{1}$ ), we have

$$
\begin{gathered}
\left.\breve{S}_{p(0)}^{+(0,2)}\left(t^{+}-t^{(0)}-\Delta t_{1}\right)\right|_{t^{+}=t_{1}}=\left.\breve{S}_{p(0)}^{(0,2)}\left(t-t^{(0)}-\Delta t_{1}\right)\right|_{t=t_{1}}=\left.\tilde{S}_{p(0)}^{(0,2)}\left(t-t^{(0)}\right)\right|_{t=t_{1}} \\
\left.\frac{\partial \breve{S}_{p(0)}^{+(0,2)}\left(t^{+}-t^{(0)}-\Delta t_{1}\right)}{\partial t^{+}}\right|_{t^{+}=t_{1}}=\left.\frac{\partial \breve{S}_{p(0)}^{(0,2)}\left(t-t^{(0)}-\Delta t_{1}\right)}{\partial t}\right|_{t=t_{1}}=\left.\frac{\partial \tilde{S}_{p(0)}^{(0,2)}\left(t-t^{(0)}\right)}{\partial t}\right|_{t=t_{1}}
\end{gathered}
$$

Thus, by the time $t=t_{3}$, the system reaches conditions identical to those the system had at the time $t=t_{1}$; i.e., the single vortex structure leaves the $\mathrm{H}_{0}$ layer. The $\mathrm{H}_{0}$ layer is entirely occupied by the recirculating zone described by the $\mathrm{Sol}_{0}$ solution. By the time $t=t_{3}$, the single vortex structure crosses the external border 
of the periphery of the recirculating zone, $r_{2}=r_{2}^{(0)}$. We assume that the time $t=t_{3}$ is the moment of separation of the single vortex structure from the recirculating zone.

Further, as previously, the periphery of the recirculating zone located in the $\mathrm{H}_{2}$ layer is replaced by a single vortex structure given by the $\mathrm{Sol}_{1}$ solution. At the same time, in the $\mathrm{H}_{1}$ layer, the recirculating zone core with the degree of excitation corresponding to the $S^{-}$state is replaced by the core with the degree of excitation corresponding to the $S^{+}$state. At the time $t=t_{3}$, if the equality (9.3a) is held, so is equality (9.3b). Then, in accordance with the criterion (7.2), the direction taken after the replacement is more preferable for the system. As at the time $t=t_{1}$, at $t=t_{3}$, a single vortex structure, in accordance with the $\mathrm{Sol}_{1}$ solution, starts to move from the outer boundary of the recirculating zone core, $r_{1}=r_{1}^{(0)}$, to the outer boundary of the recirculating zone periphery, $r_{2}=r_{2}^{(0)}$. The periodic shedding of a single vortex structure occurs with a period of

$T=t_{3}-t_{1}-\Delta t_{1}=2 t_{*}-2 t_{1}-\Delta t_{1}$. The positions of shedding vortex structures are schematically indicated by vertical lines in the lower branch in Figure 12.

It turns out that, for equality (9.3b) to be held, the degree of excitation of the recirculating zone core $\tilde{S}_{p(1)}^{(0,2)}\left(t_{1}-t^{(0)}+\Delta t_{1}\right)$ should increase with decreasing a parameter $\hat{r}_{1}^{(0)}=\hat{\tilde{C}}_{21}\left(t_{1}-t^{(0)}\right)$ that defines the position of the vortex structure at the periphery of the recirculating zone at the time of restructuring $t=t_{1}$. At $\hat{r}_{1}^{(0)}=1.580$, the degree of excitation $\tilde{S}_{p(1)}^{(0,2)}\left(t_{1}-t^{(0)}+\Delta t_{1}\right)$ of the recirculating zone core reaches its maximum, since at $\hat{r}_{1}^{(0)}=1.580$, the time $t_{1}+\Delta t_{1}$ coincides with the time of recovery $t_{*}, t_{1}+\Delta t_{1}=t_{*}$. A further decrease in the parameter $r_{1}^{(0)}$ excludes the possibility of flow restructuring, i.e., the possibility of emergence of a vortex structure at the periphery of the recirculating zone.

The time behavior of the pair entropy derivatives $\partial \tilde{S}_{p(0)}^{(0,2)}\left(t-t^{(0)}\right) / \partial t$ and $\partial\left[\breve{S}_{p(1)}^{(0,2)}\left(t-t^{(0)}\right)+\breve{S}_{p(2)}^{(1,2)}\left(t-t^{(0)}\right)\right] / \partial t=\partial \tilde{S}_{p(3)}^{(1,2)}\left(t-t^{(0)}\right) / \partial t$ is shown in Figure 10. Curve 3 corresponds to the state of the system before restructuring, $S^{-}$, curve 4 corresponds to the state of the restructured system, $S^{+}$. The curve 3 in Figure 10 is positioned slightly below curve 1. However, in the scale of Figure 10, curve 1 and 3 are indistinguishable. The time of restructuring $t=t_{1}$ corresponds to the point of intersection of curves 3 and 4 in Figure 10. At the time of restructuring $t=t_{1}$, equalities (9.3a) and (9.3b) are held simultaneously. In accordance with the criterion (7.2), the system takes the direction of evolution that is predetermined by the flow restructured at the time $t=t_{1}$.

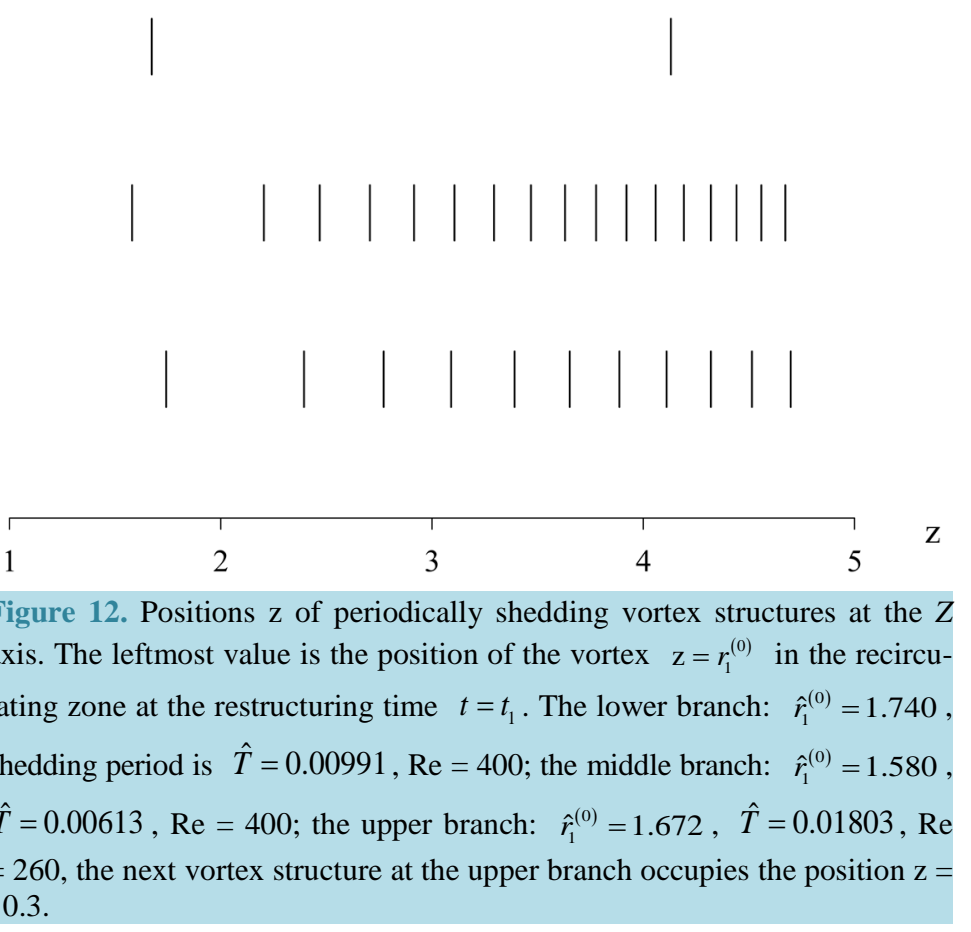


The use of the extremely low value $\hat{r}_{1}^{(0)}=1.580$ gives extremely low values of the entropy derivatives $\partial \tilde{S}_{p(0)}^{(0,2)}\left(t-t^{(0)}\right) / \partial t$ and $\partial\left[\breve{S}_{p(1)}^{(0,2)}\left(t-t^{(0)}\right)+\breve{S}_{p(2)}^{(1,2)}\left(t-t^{(0)}\right)\right] / \partial t=\partial \tilde{S}_{p(3)}^{(1,2)}\left(t-t^{(0)}\right) / \partial t$ at the time of restructuring $t=t_{1}$. The values of these derivatives at $t=t_{1}$ correspond to the intersection of curves 3 and 4 in Figure 10 . The values of $\partial \tilde{S}_{p(0)}^{(0,2)}\left(t-t^{(0)}\right) / \partial t$ and $\partial\left[\breve{S}_{p(1)}^{(0,2)}\left(t-t^{(0)}\right)+\breve{S}_{p(2)}^{(1,2)}\left(t-t^{(0)}\right)\right] / \partial t=\partial \tilde{S}_{p(3)}^{(1,2)}\left(t-t^{(0)}\right) / \partial t$ at the point of intersection of curves 3 and 4 are lower than the respective values at the point of intersection of curves 1 and 2 , for which $\hat{r}_{1}^{(0)}=1.740$. As a result, the derivative $\partial^{2}\left[\breve{S}_{p(1)}^{(0,2)}\left(t-t^{(0)}\right)+\breve{S}_{p(2)}^{(1,2)}\left(t-t^{(0)}\right)\right] / \partial t^{2}$ at $\hat{r}_{1}^{(0)}=1.580$ and $t=t_{1}$ has an extremely low value. Therefore, in accordance with the criterion (7.2), the extremely low value of $r_{1}^{(0)}$ which defines the position of a single vortex structure in the recirculating zone at the time of restructuring $t=t_{1}$ is preferable.

The use of extremely low value of $r_{1}^{(0)}$ leads to a situation where the representative point takes a minimum time to move from position of break $t=t_{*}$ to the position of separation $t=t_{3}$. The choice of an extremely low value of $r_{1}^{(0)}$ leads to an extremely low value of the period of separation of a vortex structure from the recirculating zone, $T=t_{*}-t_{1}$. In Figure 12, the vertical lines on the middle branch indicate the spatial position of periodically shedding vortex structures for an extremely low value of $r_{1}^{(0)}$. Shedding vortex structures are located extremely close to each other (middle branch).

The time behavior of the pair entropy derivatives is shown in Figure 10. Curve 5 corresponds to the $\partial \tilde{S}_{p(0)}^{(0,2)}\left(t-t^{(0)}\right) / \partial t$ function, which is calculated from the $\operatorname{Sol}_{0}$ solution at $\mathrm{Re}=260$. The curve 6 corresponds to the $\partial\left[\breve{S}_{p(1)}^{(0,2)}\left(t-t^{(0)}\right)+\breve{S}_{p(2)}^{(2,2)}\left(t-t^{(0)}\right)\right] / \partial t=\partial \tilde{S}_{p(3)}^{(1,2)}\left(t-t^{(0)}\right) / \partial t$ function, in which the $\partial \breve{S}_{p(2)}^{(2,2)}\left(t-t^{(0)}\right) / \partial t$ derivative is calculated from the $\mathrm{Sol}_{2}$ solution at $\mathrm{Re}=260$. The values of the parameters used in the calculations, $r_{1}^{(0)}, r_{2}^{(0)}, r_{1}^{(2)}, r_{2}^{(2)}$, are specified in the Figure captions.

The curve 5 corresponds to the state of the system before restructuring, $S^{-}$, while the curve 6 corresponds to the state of the restructured system, $S^{+}$. The time of restructuring $t=t_{1}$ corresponds to the point of intersection of curves 5 and 6 in Figure 10. At the time of restructuring $t=t_{1}$, the equalities (9.3a) and (9.3b) are fulfilled simultaneously. In accordance with the criterion (7.2), the system takes the direction of evolution that is set by the flow restructured at the time $t=t_{1}$.

It is found that, with increasing parameter $\hat{r}_{1}^{(0)}=\hat{\tilde{C}}_{21}\left(t_{1}-t^{(0)}\right)$, defining the position of the vortex structure in the recirculating zone at the time of restructuring $t=t_{1}$, the $\tilde{S}_{p(2)}^{(0,2)}\left(t-t^{(0)}\right)$ entropy of the recirculating zone periphery grows much faster than the $\breve{S}_{p(2)}^{(2,2)}\left(t-t^{(0)}\right)$ entropy of single vortex structure. At an extremely high value of the parameter $r_{1}^{(0)}$, the difference $\breve{S}_{p(2)}^{(2,2)}\left(t-t^{(0)}\right)-\tilde{S}_{p(2)}^{(0,2)}\left(t-t^{(0)}\right)$ is not so great as to make the equalities (9.3a) and (9.3b) simultaneously valid. The extremely high value of the parameter $r_{1}^{(0)}$ at $\operatorname{Re}=260$ is given in the Figure captions. A further increase in the parameter $r_{1}^{(0)}$ excludes the possibility of flow restructuring, i.e., the possibility of emergence of a vortex structure in the recirculating zone. The extremely high value of $r_{1}^{(0)}$ leads to the extremely low value of the period $T$ of shedding of single vortex structures from the recirculating zone core. The extremely low value of $T$ provides extremely low values of the entropy derivatives at the time of restructuring $t=t_{1}$, which are represented by the point of intersection of curves 5 and 6 in Figure 10 . Therefore, in accordance with criterion (7.2), the extremely high value of the $r_{1}^{(0)}$ parameter is selected from the spectrum of its values. The upper branch in Figure 12 shows the spatial positions of periodically shedding vortex structures for the extremely high values of $r_{1}^{(0)}$, marked by vertical lines.

The calculations have revealed the dependence of the domain of existence of the solution $\mathrm{Sol}_{1}$ on Re. At Re $=260$, the Sol ${ }_{1}$ solution exists at $\hat{C}_{21}>2.6$, here the variable $\hat{C}_{21}$ highly accurately defines the position of the vortex structure at the $Z$ axis. In this range of $\hat{C}_{21}$ values, the $\breve{S}_{p(2)}^{(1,2)}\left(t-t^{(0)}\right)$ pair entropy is not small enough to ensure that the relations (2.3) will be held. So, at $\mathrm{Re}=260$, the $\mathrm{Sol}_{2}$ solution is the only solution allowing the vortex shedding. At $\mathrm{Re}=400$, each of the solutions $\mathrm{Sol}_{1}$ and $\mathrm{Sol}_{2}$ allows the vortex shedding. Each of the solutions $\mathrm{Sol}_{1}$ and $\mathrm{Sol}_{2}$ is characterized by its own time of flow restructuring, $t=t_{1}$. Cal- 
culated from the $\mathrm{Sol}_{1}$ solution, the period $T$ of shedding of single vortex structures from the recirculating zone core is significantly smaller than the corresponding period calculated by the $\mathrm{Sol}_{2}$ solution. The entropy derivatives $\partial \tilde{S}_{p(0)}^{(0,2)}\left(t-t^{(0)}\right) / \partial t, \partial\left[\breve{S}_{p(1)}^{(0,2)}\left(t-t^{(0)}\right)+\breve{S}_{p(2)}^{(2,2)}\left(t-t^{(0)}\right)\right] / \partial t=\partial \tilde{S}_{p(3)}^{(2,2)}\left(t-t^{(0)}\right) / \partial t$ and $\partial^{2}\left[\breve{S}_{p(1)}^{(0,2)}\left(t-t^{(0)}\right)+\breve{S}_{p(2)}^{(2,2)}\left(t-t^{(0)}\right)\right] / \partial t^{2}$ at the point of restructuring $t=t_{1}$ for the Sol $_{2}$ solution are considerably higher than the respective values represented by curves 3 and 4 in Figure 10 at their point of intersection. Thus, in accordance with the criterion (7.2), the $\mathrm{Sol}_{1}$ solution is more preferable than the $\mathrm{Sol}_{2}$ solution at $\operatorname{Re}=400$.

\section{Comparison with Experiment}

All the experiments with flows past a sphere available at present are divided into two groups in the review [1]. The first group includes experiments in which a body sinks freely in a medium under the action of gravitation. Besides, the first group includes the experiments in which a hard sphere is fixed rigidly in the air or water tunnel. The second group experiments were made by towing a body through a bath filled with a liquid. The reason for such a division is as follows. The velocity of a free-stream flow can be considered as strictly uniform in none of the first group experiments. A strictly uniform profile of the flow running against a body can only be achieved in experiments of the second group [10] [11] by using a rigid support system.

According to the measurement data reported in [10] [11], the $\mathbf{U}_{0}^{\text {exp }}(\mathbf{x})$ stationary recirculating zone formed in the near wake behind a sphere at Re 10 - 20 increases as Re grows. After the attainment of the first critical Reynolds number $\mathrm{Re}_{0}^{*}$, the periphery of the recirculating zone in the near wake behind a sphere begins to pulsate periodically. The recirculating zone remains toroidal during pulsations, and its forefront is firmly fixed on the sphere. The $\mathbf{V}_{0}^{e x p}(t, \mathbf{x})$ pulsating flow remains axisymmetric.

The pulsations become increasingly well defined as Re increases, and their amplitude grows. After the passage of $\mathrm{Re}_{0}^{* *}$, the periphery of the recirculating zone begins to be periodically shed from its core and moves downstream. The shed vortex structure has the shape of ring. Separate vortex rings depart from a sphere downstream and move along the spiral path $\mathbf{W}_{0}^{e x p}(t, \mathbf{x})$ [11]. The attainment of $\operatorname{Re}_{0}^{* * * *}$ is accompanied by the change in the regime of vortex shedding from sphere. The frequency, which characterizes the vortex shedding, monotonically increases as $\mathrm{Re}$ grows starting with $\mathrm{Re}=\mathrm{Re}_{0}^{* * * *}$. The increase in the frequency causes the complete disappearance of intervals between vortex rings. Vortex rings penetrate into each other and form the $\mathbf{Q}_{0}^{e x p}(t, \mathbf{x})$ continuous spiral sheet in the wake behind a sphere [11]. According to the measurement data reported in [10], $\operatorname{Re}_{0}^{*}=130$. In [11], the $\operatorname{Re}_{0}^{*}=200$, the $\mathrm{Re}_{0}^{* *}=400$ and $\mathrm{Re}_{0}^{* * * *}=800$ values were obtained. The $\mathbf{Q}_{0}^{\exp }(t, \mathbf{x})$ continuous spiral vortex sheet was observed in [11] over the whole range of Reynolds numbers studied, up to $\mathrm{Re}=30,000$.

The simplest solution to the multimoment hydrodynamic equations gives the distribution (3.6-3.7). This distribution coincides with the Stokes solution to the classic hydrodynamics equations in the $\mathrm{Re} \ll 1$ limit. The $\mathrm{Sol}_{1}$ stationary axisymmetric solution to the S20 set of the multimoment hydrodynamic equations satisfactorily reproduces the $\mathbf{U}_{0}^{\text {exp }}(\mathbf{x})$ stable flow around a sphere. The attainment of the first critical Reynolds number $\mathrm{Re}=\mathrm{Re}_{0}^{*}=129.1$ is accompanied by the $\mathrm{Sol}_{0}$ solution stability loss. The cause for stability loss is as follows. After the passage of $\mathrm{Re}_{0}^{*}$, the entropy outflow through the surface confining the system begins to exceed the entropy production in the system. Such interpretation corresponds to the principle of retention and loss of the open system stability (6.11). The $\operatorname{Sol}_{0}$ unstable axisymmetric solution reproduces satisfactorily the $\mathbf{V}_{0}^{\text {exp }}(t, \mathbf{x})$ first unstable flow regime.

After the attainment of the second critical Reynolds number value $\mathrm{Re}=\mathrm{Re}_{0}^{* *}$, the $\mathrm{Sol}_{0}$ solution for the periphery of the recirculating zone and in the far wake is replaced by the $\mathrm{Sol}_{2}$ solution to the S22 set, which describes a vortex ring moving downstream. The cause for the replacement is that the combination of the solutions $\mathrm{Sol}_{0}$ and $\mathrm{Sol}_{2}$ becomes more preferable in comparison with the $\mathrm{Sol}_{0}$ solution. The combination of the solutions $\mathrm{Sol}_{0}$ and $\mathrm{Sol}_{2}$ provides a sharper drop in the entropy in the course of evolution than the $\mathrm{Sol}_{0}$ solution does (Figure 10). The criterion (7.2) dictates the choice of the direction of development that is given by combination of the $\mathrm{Sol}_{0}$ and $\mathrm{Sol}_{2}$ solutions. The positions of detached vortices at the $Z$ axis at $\mathrm{Re}_{0}^{1}=260$ given by the $\mathrm{Sol}_{2}$ solution, are shown in the upper branch in Figure $12\left(\mathrm{Re}_{0}^{* * *}<\mathrm{Re}_{0}^{1}<\mathrm{Re}_{0}^{* * * *}\right)$. According to the $\mathrm{Sol}_{2}$ solution, there are significant intervals between neighboring vortex rings. The combination of the solutions $\mathrm{Sol}_{0}$ and $\mathrm{Sol}_{2}$ describes the vortex street $\mathbf{W}_{0}^{\text {exp }}(t, \mathbf{x})$. 
After the attainment of the third critical Reynolds number value $\mathrm{Re}=\mathrm{Re}_{0}^{* * * *}$, the $\mathrm{Sol}_{2}$ solution at the periphery of the recirculating zone and in the far wake behind the sphere is replaced by the $\mathrm{Sol}_{1}$ solution to the S22 set, which also describes a vortex ring moving downstream. The cause for the replacement is that the combination of the solutions $\mathrm{Sol}_{0}$ and $\mathrm{Sol}_{1}$ provides a sharper decrease in the entropy in the course of evolution than the combination of the solutions $\mathrm{Sol}_{0}$ and $\mathrm{Sol}_{2}$ does (Figure 10). Criterion (7.2) dictates the choice of the direction of development that is given by combination of the $\mathrm{Sol}_{0}$ and $\mathrm{Sol}_{1}$ solutions. The positions of detached vortices at the $Z$ axis at $\mathrm{Re}_{0}^{2}=400$, given by the $\mathrm{Sol}_{1}$ solution, are shown in the middle branch of Figure 12 $\left(\operatorname{Re}_{0}^{2}>\operatorname{Re}_{0}^{* * * *}\right)$. The combination of the solutions $\mathrm{Sol}_{0}$ and $\mathrm{Sol}_{1}$ describes the vortex sheet $\mathbf{Q}_{0}^{\exp }(t, \mathbf{x})$.

The observed process of formation and separation of the vortex structure from the recirculating zone in the wake behind the sphere is shown in photographs 40 and 41 in [21]. A vortex structure arising near the surface of the sphere moves inside the core zone of the recirculating zone to its periphery, increasing in size and acquiring a definite shape. At the periphery of the recirculating zone, the vortex structure is separated from this zone and moves downstream.

In the calculated flow pattern, a formed vortex structure appears in the recirculating zone instantly at the time $t=t_{1}$. This simplification of the observed complex process of formation and separation of the vortex structure is motivated by a lack of computational resources for simulating the process of vortex shedding.

Eighteen trajectory invariants are used to generate the functions (5.1), (5.2). These invariants give the distributions of the principle hydrodynamic values (4.4)-(4.10) coupled with coefficients $\hat{C}_{i}, i=1, \cdots, 20$. Only two components coupled with the coefficients $\hat{C}_{14}$ and $\hat{C}_{20}$ form the distribution of the particle-density flux (4.8). Moreover, only one of these components coupled with the $\hat{C}_{20}$ coefficient dominates the particle density flux (4.8), reproducing recirculating zone in the near wake behind the sphere. The $F_{z}$ drag coefficient of the sphere also depends only on one component coupled with $\hat{C}_{7}: F_{z}=24 / \operatorname{Re}-16 \hat{C}_{7} / 3$. Moreover, the same $\hat{C}_{7}$ coefficient controls the pressure drop at a surface of the sphere at points $\theta=0$ and $\theta=\pi$ :

$$
p(r=a, \theta=0)-p(r=a, \theta=\pi)=-m n_{0} U_{0}^{2}\left(6 / \operatorname{Re}-4 \hat{C}_{7} / 3\right) .
$$

Further, the dominant component in the distribution (4.8) that is coupled with the $\hat{C}_{20}$ coefficient is used to reproduce a single downstream-moving vortex structure unrelated to the sphere, expression (8.1). Thus, a detailed description of the observed pattern of nucleation and separation of the vortex structure can be achieved by increasing the number of trajectory invariants involved in calculating the particle-density flux distribution. This increase is necessary both in the formulation of the set S20 describing the pulsations of the recirculating zone in the near wake and in the formulation of the set S22 describing the motion of single vortex structure outside the recirculating zone. Moreover, constructing the set of equations suitable for describing the far wake only (system S22) is barely an approximation of the calculation procedure. A further improvement necessitates combining sets S20 and S22. The solutions to the combined set must describe the movement of the single vortex structure not only in the far wake, but also in the recirculating zone. It is these solutions to the combined set of equations that must compete with the solutions to the set S20.

Forming the set of equations S20 and S22 was conducted so that these sets would be able to reproduce the time behavior of the recirculating zone and the evolution of the vortex structure separated from it in the wake of the sphere. The terms of expansion (4.2) proportional to $C_{19}, C_{20}$, and $C_{21}$ enabled us to describe the wake behind a sphere. By virtue of the proportional to $C_{19}, C_{20}$, and $c_{21}$ terms, however, yet another physically improbable vortex configurations arise in front of the sphere at its surface at a sufficiently high Re [8]. The appearance of physically improbable vortex configurations in the distribution of particle density flux (4.8) generally does not allow of using this distribution outside the wake. To obtain the distributions of the hydrodynamic values outside the wake, it is necessary to formulate a different set of the multimoment hydrodynamics equations. The boundary separating solutions to different sets should be sought based on the principle (7.3). In other words, the boundary separating the solutions should be positioned in space so as to ensure the most rapid decrease of the entropy.

In the Section 6, in calculating the pair entropy, the Sol $_{0}$ solution to the set S20 was used both in the wake behind the sphere and outside the wake. At $\mathrm{Re}$ close to $\mathrm{Re}_{0}^{*}$, physically improbable vortex configurations in front of the sphere are very weak. That is why, these configurations distort the calculation very little at $\mathrm{Re} \sim \mathrm{Re}_{0}^{*}$. Another result of going with the $\mathrm{Sol}_{0}$ solution trace beyond the wake produced divergent terms in the course of spatial integration over the volume $V$. The mergence of divergent terms forced us to restrict the in- 
tegration limits in the Section 6 to a spherical concentric layer directly adjacent to the surface of the sphere. Namely, in deriving Equation (6.6), the integration limit for indefinitely increasing terms is changed by putting $1 \leq r \leq 2, \pi \leq \theta \leq 0,2 \pi \leq \varphi \leq 0$. As revealed by calculations, the appropriate numerical coefficients in Equation (6.6) change under changes in the integration limit, however, characteristic features of variation of $\bar{S}_{p}^{(0,2)}+\delta S_{p}^{R(0,2)}(t)$ and $\partial \delta S_{p}^{R(0,2)}(t) / \partial t$ remains unchanged.

The approximate calculation of the pair entropy in Section 9 was performed within hemispherical concentric layers $\mathrm{H}_{0}, \mathrm{H}_{1}$, and $\mathrm{H}_{2}$ (Figure 9). This approximation was used because the dominant contribution to the deviation of the entropy from its equilibrium value comes from processes in the near wake behind the sphere. These are the processes of excitation of the recirculating zone and of separation of the vortex structure that appeared in this zone. The outer boundary $r_{2}$ of the layer $\mathrm{H}_{0}$ is specified by the position of the separating vortex structure at the time of its detachment, $t=t_{3}$, from the recirculating zone. Estimates show that the approximation of the boundaries of the near wake behind the sphere by the boundaries of the hemispherical layer $\mathrm{H}_{0}$ does not lead to a qualitative distortion of the results.

\section{Discussion}

Let us substitute the inequality (6.11a) into Equation (6.1) that is written in $S_{1}(t)$ terms. We obtain that the $\alpha$ state of the open system remains stable if

$$
\frac{\partial \delta S_{p}^{(\alpha)}(t)}{\partial t} \geq 0 \quad \text { for } \delta S_{p}^{(\alpha)}(t) \leq 0
$$

or, in terms of Boltzmann entropy

$$
\frac{\partial \delta S_{1}^{(\alpha)}(t)}{\partial t} \geq 0 \text { for } \delta S_{1}^{(\alpha)}(t) \leq 0
$$

The negative definite value $\delta S_{1}^{(\alpha)}(t)$ serves as Lyapunov function, and then, the first of inequalities (11.1b) is a sufficient condition for the system stability in re to $\delta S_{1}^{(\alpha)}(t) \leq 0$ fluctuations. According to the Lyapunov theorem, the condition (11.1b) is compatible with the fundamental Lyapunov definition of stability. Inequality (11.1b) makes up a formal mathematical stability criterion. A formal mathematical criterion, however, gives no way of revealing the physical cause of stability or instability of an open system.

Behind the modern concepts of open system stability is the Glansdorff-Prigogine theory [12]. This theory, in turn, is formulated in terms of nonequilibrium thermodynamics and, therefore, allows for the possibility of constructing the universal function $Z(t, \mathbf{x})$ with non-positive fluctuation at any fluctuations of hydrodynamic values

$$
Z(t, \mathbf{x})=S_{1}(t, \mathbf{x})-\frac{m n U^{2}}{2 T}
$$

The fluctuation of $Z(t, \mathbf{x})$ was used as Lyapunov function. The set of stability conditions derived in [12] in terms of this fluctuation involves inequality (1.11b) and

$$
\frac{\partial}{\partial t} \int \frac{\delta\left(m n U^{2}\right)}{2 T} \mathrm{~d} \mathbf{x} \leq 0 \quad \text { for } \int \frac{\delta\left(m n U^{2}\right)}{2 T} \mathrm{~d} \mathbf{x} \geq 0
$$

Based on inequalities (11.1b) and (11.2), P. Glansdorff and I. Prigogine formulated the condition for stability of open system state in terms of entropy production and entropy outflow [12]. They argue that the main quality controlling the stability and evolution of the system is excess of entropy production $\Delta_{I N} \delta S_{1}^{(\alpha)}(t)$. The Glansdorff-Prigogine stability condition states that for the system state to be stable, the entropy excess must be generated in the system rather than being absorbed

$$
\Delta_{I N} \delta S_{1}^{(\alpha)}(t) \geq 0 \text { for } \delta S_{1}^{(\alpha)}(t) \leq 0
$$

In accordance with Glansdorff-Prigogine theory [12], for the $\alpha$-state to be stable, the effect of excess of entropy outflow on the system must be equivalent to the effect of excess of entropy production. In other words, for the state to be stable, entropy excess must flow into the system through the confining surface, thus augmenting the entropy excess generated in the system 


$$
\Delta_{E X} \delta S_{1}^{(\alpha)}(t) \leq 0 \text { for } \delta S_{1}^{(\alpha)}(t) \leq 0
$$

Inequalities (11.3) and (11.4) supplemented with similar inequalities for the fluctuation of the $m n U^{2} / 2 T$ function (11.2) incorporating the terms of the order responsible for system stability make up the GlansdorffPrigogine stability criterion [12]. Therefore, in [12], the additional constraints (11.2) and (11.4) are imposed on stability condition (11.3).

The methods of kinetic theory and hydrodynamics amplified in this study offer a much more effective means of constructing Lyapunov function than nonequilibrium thermodynamics. In particular, there was no need in imposing constraint (11.2) on fluctuation of the $m n U^{2} / 2 T$ function. Having made sure that a positive definite fluctuation set the system on a very unlike unrealistic path, we bounded the domain of definition of $S_{1}^{(\alpha)}(t)$ by condition $\delta C_{19}^{R(0)} \geq 0$. In the bounded domain of definition, $\delta S_{1}^{(\alpha)}(t)$, as revealed by calculation, is negative definite and, therefore, can be used as a Lyapunov function. In the Glansdorff -Prigogine theory, excess of entropy outflow through the surface confining the system $\Delta_{E X} \delta S_{1}^{(\alpha)}(t)$ is treated as a quantity of secondary importance. The Glansdorff-Prigogine stability criterion was formulated, assuming that excess of entropy outflow exerted a strictly limited effect on the system, identical to the effect of excess of entropy production. The central idea of novel concept of stability is that the excess of entropy production and excess of entropy outflow are equally important. According to principle proposed in this study, the factor controlling the stability of the system is a balance of excess of entropy production and excess of entropy outflow rather than one of these quantities alone.

Let us verify that stability condition (6.11) is more general than the Glansdorff-Prigogine criterion. Indeed, if inequalities (11.3) and (11.4) are true, condition (11.1b) is surely met. If the reverse is the case (inequalities (11.3) and (11.4) fail), the system is not necessarily unstable. The excesses of entropy production $\Delta_{I N} \delta S_{1}^{(0,2)}(t=0)$ and $\Delta_{I N} \delta S_{p}^{(0,2)}(t=0)$ calculated with due regard for the terms of the order of $k n_{0} v_{0} \mathrm{Ma}^{6} / \tau_{0} \mathrm{Re}^{2}$ responsible for the system stability are plotted against Re in Figure 13. The $\Delta_{I N} \delta S_{p}^{(0,2)}(t=0)$ function is given by Equation (6.8), the $\Delta_{I N} \delta S_{1}^{(0,2)}(t=0)$ function is defined in [19]. From Figure 13 follows that $\Delta_{I N} \delta S_{1}^{(0,2)}(t=0)<0$, $\Delta_{I N} \delta S_{p}^{(0,2)}(t=0)<0$ at $\operatorname{Re}<\operatorname{Re}_{0}^{*}$. Thus, although the Glansdorff-Prigogine criterion breaks, the system is stable by virtue of relationship (6.11a) between entropy production and entropy outflow. Therefore, the Glansdorff-Prigogine stability criterion generally is incapable of interpreting hydrodynamic instability. More detailed comparison of the criterion (6.11) with the Glansdorff-Prigogine criterion was carried out in [19].

To derive the evolution criterion, the authors of [12] set up a balance equation for certain part of the total entropy of the system and differentiated it with respect to time. The equation takes into account variation of the entropy production solely under variation of generalized forces with time. The formulation of the evolution criterion was made possible by the neglect of the time differential of the excess of entropy outflow through the confining surface. There is generally no reason to disregard the excess of entropy outflow, and therefore, the Glansdorff-Prigogine evolution criterion is not universally true. The criterion states that evolution of an open system with time independent boundary conditions follows the path along which the entropy production in the system has no tendency for an increase with time

$$
\frac{\partial \Delta_{I N} \delta S_{1}^{(\alpha)}(t)}{\partial t} \leq 0
$$

The time history of $\Delta_{I N} \delta S_{p}^{R(0,1)}(t)$ of the dominating order of $k n_{0} v_{0} \mathrm{Ma}^{4} / \tau_{0} \operatorname{Re}^{2}$ is shown in Figure 14 . The excess of pair entropy $\Delta_{I N} \delta S_{p}^{R(0,1)}(t)$ is given by Equation (6.7). As revealed by calculation, the system develops in accord with inequality (11.5) at $\operatorname{Re}=129$ and $\operatorname{Re}<\operatorname{Re}_{0}^{*}$. At $\operatorname{Re}=130$ and $\operatorname{Re}>\operatorname{Re}_{0}^{*}$ (the covered Re values did not exceed 400) the Glansdorff-Prigogine evolution criterion is invalid. So, the property (11.5) is valid when system relaxes to the stable state. However, system evolution after stability loss is not accompanied by fulfillment of the property (11.5). The above formulated conclusion remains invariant in going from terms of pair entropy to terms of Boltzmann entropy.

The need to use the Glansdorff-Prigogine criterion of evolution on a search of solutions to the classic hydrodynamics equations is missing. The criterion demonstrates the fulfillment (or absence of the fulfillment) of the property (11.5) for the found solution to the hydrodynamics equations. The function of the criterion (7.2) is very significant. Criterion (7.2) chooses a direction of evolution for the system. i.e., after stability loss, the calculation of hydrodynamic values is impossible without the attraction of the criterion (7.2). 


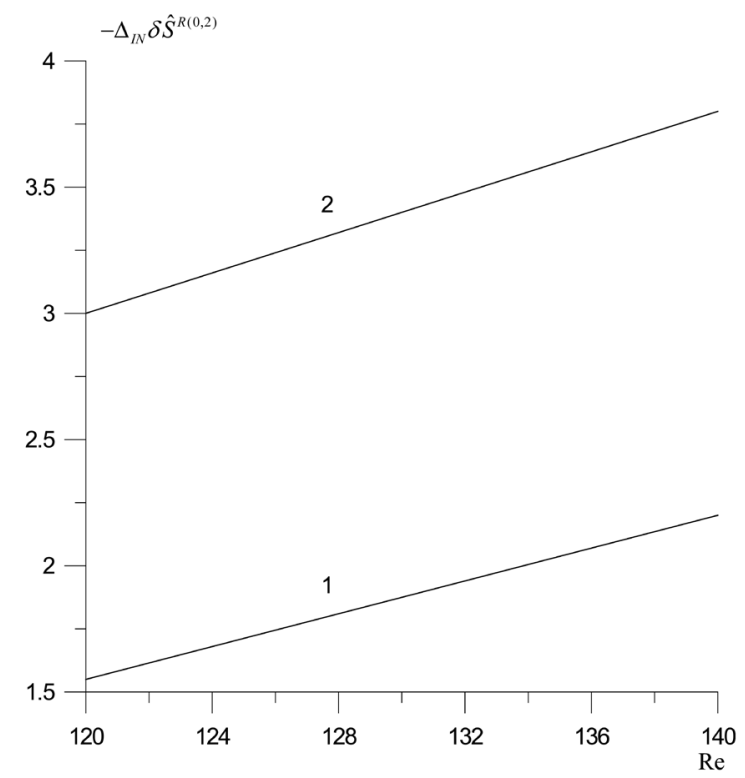

Figure 13. The excess entropy production component of the order of $k n_{0} v_{0} \mathrm{Ma}^{6} / \tau_{0} \mathrm{Re}^{2}$ responsible for instability as a function of Re. Curve 1 corresponds to the excess of the pair entropy production $-\left.\Delta_{I N} \delta \hat{S}_{p}^{R(0,2)}(t)\right|_{t=0} \cdot 10^{6}$, and curve 2, the excess of Boltzmann entropy production $-\left.\Delta_{I N} \delta \hat{S}_{1}^{R(0,2)}(t)\right|_{t=0} \cdot 10^{4}$. Entropy fluctuation at time $t=0$ is assumed to be independent of Re: $\left.\delta \hat{S}_{p}^{R(0,2)}(t)\right|_{t=0}=-1.5 \times 10^{-6}$; $\left.\delta \hat{S}_{1}^{R(0,2)}(t)\right|_{t=0}=-0.75 \times 10^{-6}$.

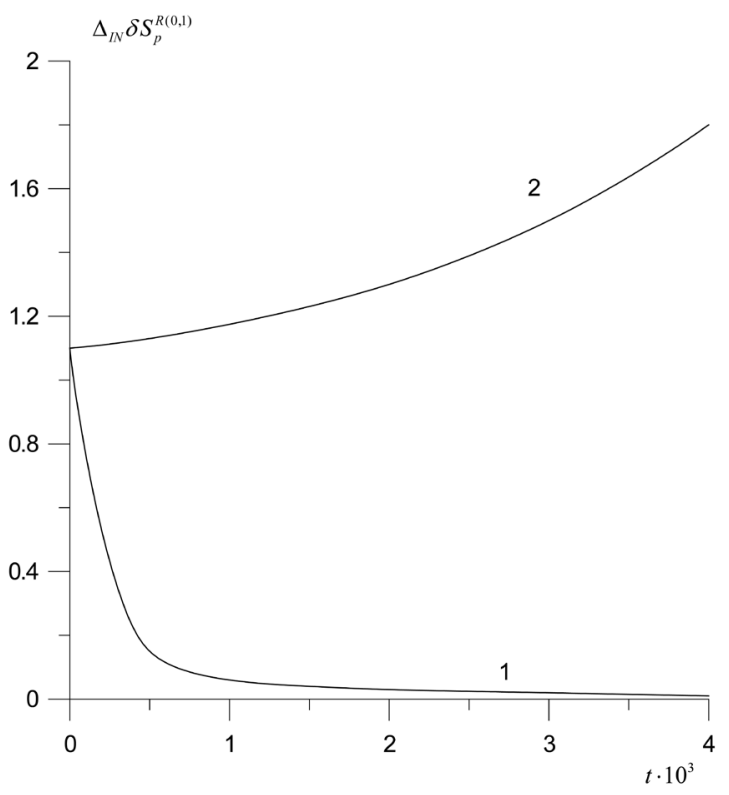

Figure 14. Time history of the $\Delta_{I N} \delta \hat{S}_{p}^{R(0,1)}(t) \cdot 10^{5}$ excess entropy production component of the dominating order of $k n_{0} v_{0} \mathrm{Ma}^{4} / \tau_{0} \mathrm{Re}^{2}$ : curve 1 corresponds to a subcritical $\operatorname{Re}$ value of $\operatorname{Re}=129$, and curve 2 , to supercritical $\operatorname{Re}$ value of $\operatorname{Re}=130$. 
Since the time of Boltzmann, the responsibility for directing the evolution of the system rests with the initial conditions realized in the system, namely the set of initial values of the coordinates and velocities of all particles [22] [23]. For a given mutual arrangement of the particles, the system evolves in the direction that we see everywhere and every second. However, there are such arrangements of particles that direct the system in an extremely unlikely, rarely realized direction. Even weak disturbance $\delta l$ of the configuration of particles $(\delta l \ll d)$ can change the direction of evolution ( $d$ is characteristic size of particles) [24].

The local pair entropy corresponding to the direct equations for pair distribution functions (1.12) from [6] and the multimoment hydrodynamics equations they yield can only be produced in the system due to binary collisions at any space point $\mathbf{x}$ and at any instant $t, \Delta_{I N} S_{p}(t, \mathbf{x}) \geq 0$. Thus, at any instant binary collisions merely rise the pair entropy of the system, $\Delta_{I N} S_{p}(t) \geq 0$. Such behavior of the entropy is in full accordance with the second law of thermodynamics [25]. The solutions to the direct multimoment hydrodynamics equations describe the direction of evolution of the system that is everywhere and every second is found in nature.

The local pair entropy corresponding to the reverse equations for the pair distribution functions (1.15) from [6] and the reverse multimoment hydrodynamics equations they yield can only be absorbed in the system due to binary collisions at any space point $\mathbf{x}$ and at any instant $t^{+}, \Delta_{I N} S_{p}^{+}\left(t^{+}, \mathbf{x}\right) \leq 0$. Thus, at any instant binary collisions absorb the pair entropy of the system, $\Delta_{I N} S_{p}^{+}\left(t^{+}\right) \leq 0$. The solutions to the reverse multimoment hydrodynamics equations describe the evolution of the system in the opposite direction, which, as is commonly believed, is extremely rare in nature.

The direct multimoment hydrodynamics equations are valid for the progressive direction of timing on the time axis pointing from the past to the future. The reverse multimoment hydrodynamics equations are valid for multimoment regressive direction of timing on the same time axis [6]. Processes occurring in nature are objective events, while the choice of the direction of timing on the time axis is subjective process. Time is counted by an observer, while processes occurring in nature absolutely insensitive to the direction in which the observer counts the time. Let two observers, agreeing upon the origin, began to observe some phenomenon. Let the first observer counts time in the regressive direction at the time axis, directed from the past to the future and let the first observer managed to describe the phenomenon he observed using the reverse equations. Let now the second observer counts time in the progressive direction at the same time axis. Then, the second observer can claim that the reverse equations described phenomena for progressive direction of timing.

This means the following. On finding the solution to the inverse equations, the first observer obtained the distribution of all hydrodynamic variables and their spatial and temporal derivatives. The first observer found agreement between the calculated and measured values. The second observer used the solution to the reverse equations to compare with his observations and, on establishing the appropriate relationship between the two time scales, found that the reverse distributions agree with the observed values in their time scale. Changing the sign of the time derivatives, the second observer also obtained an agreement with his observations. However, the second observer failed to derive direct equations that would satisfy altered distribution.

This example returns us to the Boltzmann time, when there were heated debates about the correctness of the Boltzmann equation and the H-theorem. Boltzmann opponents, E. Zermelo and J. Loschmidt, gave examples of processes that are not described by the Boltzmann equation [23] [26]. Basically, L. Boltzmann acknowledged the objections of opponents, agreeing with the existence of processes that can not be governed by his theory. However, L. Boltzmann argued that such processes was extremely unlikely, i.e., impracticable [22]. Probably, at a weak deviation of the system state from the state of thermodynamic equilibrium, as predicted L. Boltzmann, conditions guided a system in the unlikely direction are extremely rare. However, as the degree of nonequilibrium increases the probability of occurrence of such conditions may grow. The penetration into the instability field confirmed this assumption. It turned out that the motion of unstable system along the unlikely direction becomes an event that repeats periodically.

\section{Conclusions}

The experiment records two stable stationary medium states represented by the $\mathbf{U}_{0}^{\exp }(\mathbf{x})$ and $\mathbf{U}_{1}^{\exp }(\mathbf{x})$ velocity distributions, and a stable state of the central type with the $\mathbf{U}_{2}^{\text {exp }}(t, \mathbf{x})$ velocity distribution. Each of these three stable flows begins to develop in its own direction qualitatively different from other flows when it loses stability. The development occurs through a sequence of regular nonstationary periodic states schematically shown in Figure 1 from [3]. Each of these three experimentally observed directions inevitably reaches the periodic vortex 
shedding mode. Vortex shedding along each of three directions is characterized by its own characteristic features intrinsic in it. However, irrespective of the direction selected experimentally, periodic vortex shedding is obligatory, well defined, and fairly prolonged along Reynolds numbers mode of the development of a turbulent process. Experiment records six vortex shedding modes, and one pulsation mode along the three directions of instability development. The development of the $\mathbf{U}_{0}^{\text {exp }}(\mathbf{x})$ ground flow occurs through the $\mathbf{V}_{0}^{\text {exp }}(t, \mathbf{x})$ pulsating regime, and further, through the vortex shedding regimes $\mathbf{W}_{0}^{\exp }(t, \mathbf{x})$ and $\mathbf{Q}_{0}^{\exp }(t, \mathbf{x})$.

The direct numerical integration of Navier-Stokes equations in the problem on a flow around a solid sphere at rest was performed by various numerical methods. Nevertheless, the results of all these numerical experiments were absolutely identical (see review [1]). Calculations find two stable stationary solutions, $\mathbf{U}_{0}^{\text {cal }}(\mathbf{x})$ and $\mathbf{U}_{1}^{\text {cal }}(\mathbf{x})$ and a nonstationary stable solution, $\mathbf{U}_{2}^{\text {cal }}(t, \mathbf{x})$. Apart from the $\mathbf{U}_{0}^{\text {cal }}(\mathbf{x}), \mathbf{U}_{1}^{\text {cal }}(\mathbf{x})$, and $\mathbf{U}_{2}^{\text {cal }}(t, \mathbf{x})$ solutions, the Navier-Stokes equations only have a multiperiodic, that is, essentially chaotic, solution $\mathbf{U}_{3}^{\text {cal }}(t, \mathbf{x})$. The $\mathbf{U}_{0}^{\text {cal }}(\mathbf{x})$, $\mathbf{U}_{1}^{\text {cal }}(\mathbf{x})$, and $\mathbf{U}_{2}^{\text {cal }}(t, \mathbf{x})$ solutions satisfactorily reproduce $\mathbf{U}_{0}^{\exp }(\mathbf{x}), \mathbf{U}_{1}^{\text {exp }}(\mathbf{x})$, and $\mathbf{U}_{2}^{\text {exp }}(t, \mathbf{x})$ stable flows.

According to calculations, the development of instability occurs in strict correspondence to the classic Landau-Hopf scenario [14]. After first critical Reynolds number value is reached, the $\mathbf{U}_{0}^{\text {cal }}(\mathbf{x})$ solution loses stability and bifurcates to the $\mathbf{U}_{1}^{\text {cal }}(\mathbf{x})$ solution (regular bifurcation). The $\mathbf{U}_{1}^{\text {cal }}(\mathbf{x})$ solution, after it loses stability, bifurcates to the $\mathbf{U}_{2}^{\text {cal }}(t, \mathbf{x})$ limiting cycle (the Hopf bifurcation). After next critical Reynolds number value is reached, the $\mathbf{U}_{2}^{\text {cal }}(t, \mathbf{x})$ limiting cycle loses stability and is substituted by the $\mathbf{U}_{3}^{\text {cal }}(t, \mathbf{x})$ chaotic solution. It follows that, according to the Landau-Hopf scenario, the system, after it loses stability, inevitably reaches a new stable position and exercises either periodic or chaotic motion about it [27]. So, calculations cannot put anything in correspondence to seven of ten experimentally observed modes schematically shown in Figure 1 from [3]. In particular, to periodic pulsation of the recirculating zone not accompanied by vortex shedding, $\mathbf{V}_{0}^{\text {exp }}(t, \mathbf{x})$, and two periodic vortex shedding modes, $\mathbf{W}_{0}^{\exp }(t, \mathbf{x})$ and $\mathbf{Q}_{0}^{\exp }(t, \mathbf{x})$.

The ideas of experiment on the nature of the phenomenon of vortex shedding fundamentally disagree with the concepts of classic hydrodynamics [14]. In accordance with experiment, a system, when loses stability after the attainment of a certain critical Reynolds number value, remains further in unstable state. One unstable regime is replaced by another unstable regime as Re grows. Experiment interprets the observed phenomenon of vortex shedding as the von Karman instability, or as the Kelvin-Helmholtz instability. By definition [28], the instability occurs only during the flow transformation, i.e., the time intervals when one solution loses its stability and another stable solution is saturated. The process of saturation of new stable state is extremely limited in time and non-periodic. i.e., the bulk of time the system is at a stable state. Time-limited non-periodic process of saturation can in no way correlate with the strictly periodic vortex shedding phenomenon, which, generally, is not limited in time. Thus, the solutions to the classic hydrodynamics equations are incapable of corresponding to ideas of experiment on unstable nature of the vortex shedding phenomenon. Classic hydrodynamics can put only stable solutions in correspondence with observed vortex shedding modes. In particular, in problem on flow around a sphere the $\mathbf{U}_{2}^{\text {cal }}(t, \mathbf{x})$ limiting cycle is the only case where it is possible to correlate the observed vortex shedding from a sphere to calculation result. However, numerous attempts to find a vortex street in the $\mathbf{U}_{2}^{\text {cal }}(t, \mathbf{x})$ far wake behind a body failed [1] [2] [21].

The multimoment hydrodynamics confirms the ideas of experiment on unstable nature of the phenomenon of vortex shedding. The crossing of the first critical Reynolds number value $\mathrm{Re}_{0}^{*}$ is accompanied by the stability loss. The system loses its stability when entropy produced in the system can not compensate entropy outflow through the surface confining the system. Such interpretation follows directly from the principle of retention and loss of the open system stability formulated in Section 6. In accordance with solutions to the multimoment hydrodynamics equations, the system, when loses its stability, remains further unstable. One unstable flow is replaced by another unstable flow as Re grows. The replacement of one unstable flow regime by another unstable regime is governed the tendency of the system to discover the fastest path to depart from the state of statistical equilibrium. This striving follows directly from the evolution criterion formulated in Section 7.

Figure 8 demonstrates the behavior of pair entropy of the system losing its stability. According to the $\mathrm{Sol}_{0}$ solution, pair entropy describes periodic pulsations of the recirculating zone in the wake behind a sphere. Starting with $t=0$, pair entropy begins to decay monotonously. The monotonous decay is replaced by surge. However, the sharp decrease of entropy is not unlimited. At $t=t_{*}$, the decrease of entropy finishes. Starting with $t=t_{*}$, pair entropy begins to increase up to time $t=2 t_{*}$. At $t=2 t_{*}$, the system returns to its original position. Pair entropy begins to decay again starting with $t=2 t_{*}$. The process is repeated with the period $t=2 t_{*}$.

A similar behavior demonstrates pair entropy, which is associated with periodic vortex shedding, Figure 9 . 
The sharp decrease of entropy is completed at $t=t_{*}$. Starting with $t=t_{*}$, pair entropy begins to increase up to time $t=t_{3}$. At $t=t_{3}$, the system reaches the position that it occupied at $t=t_{1}$. Pair entropy begins to decay again starting with $t=t_{3}$. The process is repeated with the period $T=t_{3}-t_{1}-\Delta t_{1}$.

The cut-off of the $\mathrm{Sol}_{0}$ solution is the reason for the completion of the sharp decrease of pair entropy at time $t=t_{*}$. It turned out that at $t=t_{*}$ the multimoment hydrodynamics equations [5] become unsuitable for modeling evolution of the system. However, in the neighborhood of the cut-off point there is a solution to the reverse multimoment hydrodynamics equations [6]. The solution to the reverse equations changes the direction of evolution, striving the system to the state of statistical equilibrium. The pair entropy corresponding to the direct multimoment hydrodynamics equations can only be produced in the system due to binary collisions at each time point. Such behavior of the entropy is in full accordance with the second law of thermodynamics. The pair entropy corresponding to the reverse multimoment hydrodynamics equations can only be absorbed in the system due to binary collisions at each time point. The solutions to the reverse multimoment hydrodynamics equations describe the evolution of the system in the opposite direction, which, as is commonly believed, is extremely rare in nature.

At his time, Boltzmann, defending his point of view in disputes with opponents, suggested that the exclusive conditions that guide the system in a highly unlikely direction arise very rarely. Apparently, Boltzmann's assumption is correct for a weak deviation of the system from the state of statistical equilibrium. However, after crossing the border of the instability field, exclusive conditions arise with periodic regularity. This regularity manifests itself in each of three unstable modes that represent the flows $\mathbf{V}_{0}^{\exp }(t, \mathbf{x}), \mathbf{W}_{0}^{\exp }(t, \mathbf{x})$, and $\mathbf{Q}_{0}^{\exp }(t, \mathbf{x})$.

The tendency of an unstable physical system to find the fastest path to recede from the state of statistical equilibrium does not lead the system to infinity. At time $t=t_{*}$, the entropy stops decreasing. The multimoment hydrodynamics equations are unable to provide solutions that would continue to divert the system from the state of statistical equilibrium. Time intervals during which the system moves away from the state of statistical equilibrium are periodically followed by intervals within which the system tends to equilibrium. It is this periodicity that permits to interpret vortex shedding, a graphic example of periodic unstable phenomena.

\section{References}

[1] Lebed, I.V. and Umanskii, S.Y. (2007) The Appearance and Development of Turbulence in a Flow Past a Sphere: Problems and the Existing Approaches to Their Solution. Russian Journal of Physical Chemistry B, 1, 52-73.

[2] Lebed, I.V. and Umanskii, S.Y. (2012) On the Possibility of Improving Classic Hydrodynamics Equations by an Increase in the Number of Hydrodynamic Values. Russian Journal of Physical Chemistry B, 6, 149-162.

[3] Lebed, I.V. (2013) About the Prospects for Passage to Instability. Open Journal of Fluid Dynamics, 3, $214-229$. http://dx.doi.org/10.4236/ojfd.2013.33027

[4] Lebed, I.V. (2002) On the Inapplicability of Navier-Stokes Equations to Interpreting the Turbulence. Physica A, 315, 228-235. http://dx.doi.org/10.1016/S0378-4371(02)01254-2

[5] Lebed, I.V. (1996) Method of Two-Particle Distribution Functions. Hydrodynamic Equations. Chemical Physics Reports, 15, 861-883.

[6] Lebed, I.V. (1996) Hydrodynamic Equations Stemming from Two Particle Distributions in the Limit of Weak NonEquilibrium. Analysis of Invertibility of Equations. Chemical Physics Reports, 15, 1725-1750.

[7] Loitsyanskii, L.G. (1966) Mechanics of Liquids and Gases. Pergamon, Oxford.

[8] Lebed, I.V. (1997) The Method of Pair Functions as Applied to the Problem of a Flow around a Quiescent Solid Sphere. Chemical Physics Reports, 16, 1263-1301.

[9] Tikhonov, A.N. and Samarskii, A.A. (1953) Equations of Mathematical Physics. Gostechizdat, Moscow.

[10] Taneda, S. (1956) Experimental Investigation of the Wake behind a Sphere at Low Reynolds Numbers. Journal of the Physical Society of Japan, 11, 1104-1108. http://dx.doi.org/10.1143/JPSJ.11.1104

[11] Chomaz, J.M., Bonneton, P. and Hopfinger, E.J. (1993) The Structure of the Near Wake of a Sphere Moving Horizontally in a Stratified Fluid. Journal of Fluid Mechanics, 254, 1-21. http://dx.doi.org/10.1017/S0022112093002009

[12] Glansdorff, P. and Prigogine, I. (1971) Thermodynamic Theory of Structure, Stability and Fluctuations. Wiley-Interscience, A Division of John Wiley \& Sons, Ltd., London.

[13] Prigogine, I. (1980) From Being to Becoming: Time and Complexity in the Physical Sciences. W.H. Freeman and Company, San Francisco.

[14] Schuster, H.G. (1984) Deterministic Chaos. Physik_Verlag, Weinheim. 
[15] Zubarev, D.N. (1971) Nonequilibrium Statistical Thremodynamics. Nauka, Moscow.

[16] Landau, L.D. and Lifshitz, E.M. (1976) Course of Theoretical Physics. Vol. 5, Statistical Physics, Part 1, Nauka, Moscow.

[17] Lifshitz, E.M. and Pitaevskii, L.P. (1980) Course of Theoretical Physics. Vol. 9, Statistical Physics, Part 2, Pergamon, New York.

[18] Ferziger, J.H. and Kaper, H.G. (1972) Mathematical Theory of Transport Processes in Gases. North-Holland Publishing Company, Amsterdam.

[19] Lebed, I.V. (1998) About the Behavior of the Entropy of a Gas Flow Losing Its Stability. Chemical Physics Reports, 17, 411-439.

[20] Lebed, I.V. (2014) Development of Instability in the Problem of Flow around a Sphere. Russian Journal of Physical Chemistry B, 8, 240-253.

[21] Johnson, T.A. and Patel, V.S. (1999) Flow Past a Sphere Up to a Reynolds Number of 300. Journal of Fluid Mechanics, 378, 19-70. http://dx.doi.org/10.1017/S0022112098003206

[22] Boltzmann, L. (1896) Entgegnung auf die Wärmetheoretischen Betrachtungen des Hrn. Zermelo. Ann. Phys. Chem., 57, 773-784.

[23] Zermelo, E. (1896) Uber einen Satz der Dynamik and die Mechanische Wärmetheorie. Ann. Phys. Chem., 57, $485-494$.

[24] Orban, J. and Bellemance, A. (1967) Velocity-Inversion and Irreversibility in a Dilute Gas of Hard Discs. Physics Letters A, 24, 620-621. http://dx.doi.org/10.1016/0375-9601(67)90651-2

[25] Liboff, R.L. (1969) Introduction to the Theory of Kinetic Equations. Willey, New York.

[26] Loschmidt, J. (1876) Uber den Zustand des Wärmegleichgewichtes eines Systems von Körpern mit Rücksicht auf die Schwerkraft. Wien. Ber., 73, 128-142.

[27] Tomboulides, A.G. and Orszag, S.A. (2000) Numerical Investigation of Transitional and Weak Turbulent Flow Past a Sphere. Journal of Fluid Mechanics, 416, 45-73. http://dx.doi.org/10.1017/S0022112000008880

[28] Hannemann, K. and Oertel Jr., H. (1989) Numerical Simulation of the Absolutely and Convectively Unstable Wake. Journal of Fluid Mechanics, 199, 55-88. http://dx.doi.org/10.1017/S0022112089000297

[29] Isihara, A. (1971) Statistical Physics. Academic Press, New York.

[30] Grad, H. (1949) About Kinetic Theory of Rarefied Gases. Communications on Pure and Applied Mathematics, 2, 331407. http://dx.doi.org/10.1002/cpa.3160020403 


\section{Appendix}

Suppose that $f_{p}^{\text {app }}(t, \mathbf{x}, \mathbf{G}, \mathbf{v})$ and $f_{p p}^{\text {app }}\left(t, \mathbf{x}, \boldsymbol{\xi}_{1}, \boldsymbol{\xi}_{2}\right)$ are the pair and paired-particle distribution functions corresponding to approaching particles [5]. The correlation between $f_{p}^{\text {app }}(t, \mathbf{x}, \mathbf{G}, \mathbf{v})$ and a one-particle distribution function $f_{1}\left(t, \mathbf{x}, \boldsymbol{\xi}_{1}\right)$ was established in [5] using $f_{p p}^{\text {app }}\left(t, \mathbf{x}, \boldsymbol{\xi}_{1}, \boldsymbol{\xi}_{2}\right)$

$$
\begin{gathered}
f_{1}\left(t, \mathbf{x}, \boldsymbol{\xi}_{1}\right)=\int f_{p p}^{\mathrm{app}}\left(t, \mathbf{x}, \boldsymbol{\xi}_{1}, \boldsymbol{\xi}_{2}\right) \mathrm{d} \boldsymbol{\xi}_{2} \\
f_{1}\left(t, \mathbf{x}, \boldsymbol{\xi}_{1}\right)=2 \int f_{p}^{\mathrm{app}}(t, \mathbf{x}, \mathbf{G}, \mathbf{v}) \mathrm{d} \mathbf{G d} \mathbf{v}
\end{gathered}
$$

Then from the Gibbs inequality [29] we obtain

$$
\begin{aligned}
& \int f_{p p}^{\text {app }} \ln \left(f_{p p}^{\text {app }}\right) \mathrm{d} \xi_{1} \mathrm{~d} \xi_{2} \geq \int f_{p p}^{\text {app }} \ln \left(2 f_{p p}^{\text {app }}\right) \mathrm{d} \xi_{1} \mathrm{~d} \xi_{2} \\
& \int f_{p p}^{\text {app }} \ln \left(f_{p p}^{\text {app }}\right) \mathrm{d} \xi_{1} \mathrm{~d} \xi_{2} \geq \int f_{1} \ln \left(f_{1} / V_{\xi}\right) \mathrm{d} \xi_{1}
\end{aligned}
$$

Let us set up the $\bar{S}_{p}(t, \mathbf{x})$ and $\bar{S}_{p p}(t, \mathbf{x})$ functions as follows

$$
\begin{aligned}
& \bar{S}_{p}(t, \mathbf{x})=-k \int f_{p}^{\text {app }} \ln \left(f_{p}^{\text {app }}\right) \mathrm{d} \mathbf{G} \mathrm{d} \mathbf{v} \\
& \bar{S}_{p p}(t, \mathbf{x})=-k \int f_{p p}^{\text {app }} \ln \left(f_{p p}^{\text {app }}\right) \mathrm{d} \xi_{1} \mathrm{~d} \xi_{2}
\end{aligned}
$$

Integrals with respect to velocity in expressions (A.1, A.2) are taken over a three-dimensional volume $V_{\xi}$.

$$
\begin{aligned}
& \bar{S}_{p}(t)=\int \bar{S}_{p}(t, \mathbf{x}) \mathrm{d} \mathbf{x} \\
& \bar{S}_{p p}(t)=\int \bar{S}_{p p}(t, \mathbf{x}) \mathrm{d} \mathbf{x}
\end{aligned}
$$

Spatial integration (A.4) is carried out over the physical volume of the system $V$.

$$
\begin{aligned}
& \bar{S}_{1}(t, \mathbf{x})=-k \int f_{1} \ln \left(f_{1}\right) \mathrm{d} \xi_{1} \\
& \bar{S}_{1}(t)=\int \bar{S}_{1}(t, \mathbf{x}) \mathrm{d} \mathbf{x}
\end{aligned}
$$

Here, $S_{1}$ is the Boltzmann entropy. By virtue of inequalities (A.2) we obtain

$$
\begin{aligned}
& \bar{S}_{p p}(t) \leq \bar{S}_{1}(t)+N \ln V_{\xi} \\
& \bar{S}_{p p}(t) \leq 2 \bar{S}_{p}(t)-N \ln 2
\end{aligned}
$$

The term $N \ln V_{\xi}$ in inequality (A.6) brings $\bar{S}_{p p}(t)$ and $\bar{S}_{1}(t)$ to the same dimensionality, and $N$ is a total number of particles in the system. Thus, $\bar{S}_{p p}^{p p}(t)$ is the lower bound of both $\bar{S}_{1}(t)$ and $\bar{S}_{p}(t)$. It is well known that Boltzmann entropy $\bar{S}_{1}(t)$ conveys the meaning of a volume occupied by the system in the $\Gamma$-space [18]. The possibility of comparing $\bar{S}_{p p}(t), \bar{S}_{p}(t)$, and $\bar{S}_{1}(t)$ using relationships (A.6) implies that $\bar{S}_{p p}(t)$ and $\bar{S}_{p}(t)$ have the same physical meaning as the Boltzmann entropy. The physical meaning of $\bar{S}_{p p}(t)$ and $\bar{S}_{p}(t)$ can also be elucidated in a conventional way [18]. Functions $\bar{S}_{p p}(t), \bar{S}_{p}(t)$, and $\bar{S}_{1}(t)$ approximate the total entropy of the system to different accuracies. If $\bar{S}_{p}(t)$ is termed the pair entropy of the system, $\bar{S}_{p}(t, \mathbf{x})$ is its local pair entropy.

When calculating $\bar{S}_{p}(t, \mathbf{x}), \quad f_{p}^{\text {app }}(t, \mathbf{x}, \mathbf{G}, \mathbf{v})$ is expanded in terms of a double set of Hermitian polynomials (see Equation (29) in [5]). Furthermore, let $f_{p}^{\text {app }}(t, \mathbf{x}, \mathbf{G}, \mathbf{v})$ be equal to approximations $(31,34)$ with coefficients (57) from [5], composed on seven principle hydrodynamic values $\left(n(t, \mathbf{x}), \mathbf{U}(t, \mathbf{x}), p^{v}(t, \mathbf{x}), p^{G}(t, \mathbf{x})\right.$, $p_{i j}^{G}(t, \mathbf{x}), \mathbf{q}^{v}(t, \mathbf{x})$, and $\left.\mathbf{q}^{G}(t, \mathbf{x})\right)$. The so composed $f_{p}^{\text {app }}(t, \mathbf{x}, \mathbf{G}, \mathbf{v})$ function is then substituted into Equation (A.3), and the resulting expression for $\bar{S}_{p}(t, \mathbf{x})$ is truncated to the first few non-vanishing terms

$$
\bar{S}_{p}(t, \mathbf{x})=-\frac{k n}{2} \ln \left[\frac{n}{2}\left(\frac{m}{2 \pi k T^{G}}\right)^{3 / 2}\left(\frac{m}{2 \pi k T^{V}}\right)^{3 / 2}\right]+\frac{3 k n}{2}-\frac{k n}{8}\left(\frac{p_{i j}^{G}}{p^{G}}\right)^{2}
$$

Following the Grad's procedure for calculating $\bar{S}_{1}(t, \mathbf{x})$ [30], let us expend $f_{1}\left(t, \mathbf{x}, \xi_{1}\right)$ into a series in terms of Hermitian polynomials. The hydrodynamic values appearing as coefficients in this expansion are reasonably presented in the form of linear combinations of $G$ - and $v$-components, as was done in [5]. Substituting the so formulated $f_{1}\left(t, \mathbf{x}, \xi_{1}\right)$ function into Equation (A.5) yields the expression for $\bar{S}_{1}(t, \mathbf{x})$, in which only the 
first non-vanishing terms are retained

$$
\bar{S}_{1}(t, \mathbf{x})=-k n \ln \left[n\left(\frac{m}{2 \pi k T}\right)^{3 / 2}\right]+\frac{3 k n}{2}-\frac{k n}{16}\left(\frac{p_{i j}^{G}}{p}\right)^{2}-\frac{k n p_{i j}^{G} p_{i j}^{v}}{8 p^{2}}
$$

In analyzing the evolution of the pair entropy $\bar{S}_{p}(t, \mathbf{x})$ with space and time we shall proceed from Equation (28b) given in [5]. Multiplying this equation by $\left(-k-k \ln f_{p}^{\text {app }}(t, \mathbf{x}, \mathbf{G}, \mathbf{v})\right)$ and integrating the result with respect to velocities yield

$$
\frac{\partial \bar{S}_{p}(t, \mathbf{x})}{\partial t}+\frac{\partial \overline{\mathbf{S}}_{p}(t, \mathbf{x})}{\partial \mathbf{x}}=\Delta_{I N} \bar{S}_{p}(t, \mathbf{x})
$$

The expressions for the components of the left-hand side of Equation (A.9) are written in [19]. The expression for the pair entropy production in binary collisions $\Delta_{I N} \bar{S}_{p}(t, \mathbf{x})$ assumes the form

$$
\begin{gathered}
\Delta_{I N} \bar{S}_{p}(t, \mathbf{x})=\frac{k n\left(p_{i j}^{v}\right)^{2}}{\tau\left(p^{v}\right)^{2}}+\frac{24 k m n^{2}\left(q_{i}^{G v}\right)^{2}}{5 \tau p^{G}\left(p^{v}\right)^{2}} \\
\Delta_{I N} \bar{S}_{p}(t, \mathbf{x}) \geq 0
\end{gathered}
$$

Inequality (A.10b) implies that $\Delta_{I N} \bar{S}_{p}(t, \mathbf{x})$ is non-negative at any space point $\mathbf{x}$ and at any instant $t$. Thus, binary collisions merely raise the pair entropy of the system. Since the Boltzmann's time, this result constitutes the essence of the $H$-theorem.

The space and time evolution of the Boltzmann entropy $\bar{S}_{1}(t, \mathbf{x})$ will be inferred from Equation (A.2b) derived in [5]. Integrating Equation (A.2b) from [5] with respect to $\mathbf{v}$ and taking advantage of Equations (18b) and (19.b) from [5], we obtain the equation for $f_{1}\left(t, \mathbf{x}, \xi_{1}\right)$ with the collision integral unrelated to the Boltzmann hypothesis of molecular chaos. Let us multiply the resulting equation by $\left(-k-k \ln f_{1}\left(t, \mathbf{x}, \xi_{1}\right)\right)$ and integrate it with respect to $\xi_{1}$. We obtain

$$
\frac{\partial \bar{S}_{1}(t, \mathbf{x})}{\partial t}+\frac{\partial \overline{\mathbf{S}}_{1}(t, \mathbf{x})}{\partial \mathbf{x}}=\Delta_{I N} \bar{S}_{1}(t, \mathbf{x})
$$

The expressions for the components of the left-hand side of Equation (A.11) are written in [19]. The expression for the Boltzmann entropy production in binary collisions $\Delta_{I N} \bar{S}_{1}(t, \mathbf{x})$ assumes the form

$$
\Delta_{I N} \bar{S}_{1}(t, \mathbf{x})=\frac{k n p_{i j} p_{i j}^{v}}{2 \tau p^{2}}+\frac{4 k m n^{2} q_{i} q_{i}^{G v}}{5 \tau p^{3}}-\frac{4 n^{2} p_{i j}^{G}\left(p_{k j}^{G}+2 p_{k j}^{v}\right) p_{i k}^{v}}{8 \tau p^{3}}
$$

The study undertaken in [6] has revealed that in some special cases it is possible to establish linear relations between $p_{i j}$ and $p_{i j}^{v}$ and between $q_{i}$ and $q_{i}^{G v}$. In particular, $p_{i j}=p_{i j}^{v}$ in the limit $\operatorname{Re} \rightarrow 0$ [6]. In the limit $\operatorname{Re} \rightarrow 0$, the second and the third terms of the right-hand side of Equation (A.12) are negligibly small compared to the first term. In this case

$$
\Delta_{I N} \bar{S}_{1}(t, \mathbf{x}) \stackrel{\mathrm{Re} \rightarrow 0}{\longrightarrow} \Delta_{I N} \bar{S}_{B}(t, \mathbf{x})=\frac{k n\left(p_{i j}\right)^{2}}{2 \tau p^{2}} \quad \Delta_{I N} \bar{S}_{B}(t, \mathbf{x}) \geq 0
$$

It does not generally follow from Equation (A.12) that $\Delta_{I N} \bar{S}_{1}(t, \mathbf{x})$ is non-negative value. The property (A.10) of pair entropy is the main reason why the pair entropy $\bar{S}_{p}(t, \mathbf{x})$ should be preferred to Boltzmann entropy $\bar{S}_{1}(t, \mathbf{x})$ when interpreting non-equilibrium phenomena. The tendency of the Boltzmann entropy of a gas system to build up in binary collisions was inferred from the Boltzmann equation based on the molecular chaos hypothesis [18]. If the molecular chaos hypothesis is abandoned, this tendency generally disappears. From Equation (A.13) follows that binary particle collisions produce Boltzmann entropy at any space point $\mathbf{x}$ and at any instant $t$, only if gas is close to statistical equilibrium.

Integrating Equation (A.9) with respect to $\mathbf{x}$ over the volume of the system $V$ yields

$$
\frac{\partial \bar{S}_{p}(t)}{\partial t}+\Delta_{E X} \bar{S}_{p}(t)=\Delta_{I N} \bar{S}_{p}(t) \quad \Delta_{E X} \bar{S}_{p}(t)=\oint \overline{\mathbf{S}}_{p}(t, \mathbf{x}) \mathrm{d} \boldsymbol{\Phi}
$$


The second term of the left-hand side of Equation (A.14) is integrated over surface $\Phi$ confining volume $V$. In virtue of inequality (A.10b), the right-hand side of Equation (A.14) is non-negative. Pair entropy $\bar{S}_{p}(t, \mathbf{x})$ is defined in terms of pair distribution function labeled by app superscript. In the derivation of Equation (A.9), the multimoment hydrodynamics Equations (54)-(56) from [5] supplemented with direct expressions (53) from [5], are used. Equation (A.14) is valid only in the direction of increasing time. The positive direction of the time axis runs from the past to the future [6].

Pair entropy $\bar{S}_{p}^{+}\left(t^{+}, \mathbf{x}\right)$ is defined in terms of pair distribution function labeled by div superscript. In analyzing the evolution of the pair entropy $\bar{S}_{p}^{+}\left(t^{+}, \mathbf{x}\right)$ with space and time we shall proceed from Equation (28a) given in [5]. We obtain

$$
\frac{\partial \bar{S}_{p}^{+}\left(t^{+}\right)}{\partial t^{+}}+\Delta_{E X} \bar{S}_{p}^{+}\left(t^{+}\right)=\Delta_{I N} \bar{S}_{p}^{+}\left(t^{+}\right)
$$

In the derivation of Equation (A.15), the multimoment hydrodynamics Equations (54)-(56) from [5] supplemented with reverse expressions (1.17) from [6], are used. The expression for $\bar{S}_{p}^{+}\left(t^{+}, \mathbf{x}\right)$ is given by Equation (A.7). The expression for $\Delta_{I N} \bar{S}_{p}^{+}\left(t^{+}, \mathbf{x}\right)$ differs from its counterpart (the expression (A.10a) for $\Delta_{I N} \bar{S}_{p}(t, \mathbf{x})$ ) by the sign. That is why, at any space point $\mathbf{x}$ and at any instant $t^{+}$the $\Delta_{I N} \bar{S}_{p}^{+(0)}\left(t^{+}, \mathbf{x}\right)$ function is non-positive. i.e., binary particle collisions absorb the pair entropy at any space point $\mathbf{x}$ and at any instant $t^{+}$, and right-hand side of Equation (A.15) is non-positive. Equation (A.14) is valid only in the direction of decreasing time. The positive direction of the time axis runs from the past to the future [6]. 